\title{
Passgenauigkeit von Kronen nach optischer Abformung im Vergleich mit konventionellen Verfahren
}

Inaugural-Dissertation

zur Erlangung des Grades eines Doktors der Zahnmedizin

des Fachbereichs Medizin

der Justus-Liebig-Universität Gießen

vorgelegt von Brückel, Cora

aus Gießen

Gießen 2012 
Aus dem Medizinischen Zentrum für Zahn-, Mund- und Kieferheilkunde

Poliklinik für Zahnärztliche Prothetik

der Universitätsklinikum Gießen und Marburg GmbH

Standort Gießen

Direktor: Prof. Dr. Bernd Wöstmann

Gutachter: Prof. Dr. B. Wöstmann

Gutachter: Prof. Dr. D. Heidemann

Tag der Disputation: 13.12.2012 
Meinen Eltern

meinen Großeltern

und meinem Freund Stephan gewidmet 


\section{Inhaltsverzeichnis}

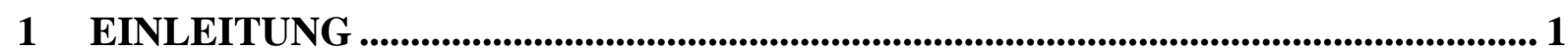

2 LITERATURÜBERSICHT ..................................................................................... 3

2.1 Überblick über die gängigen Abformmaterialien ................................................ 3

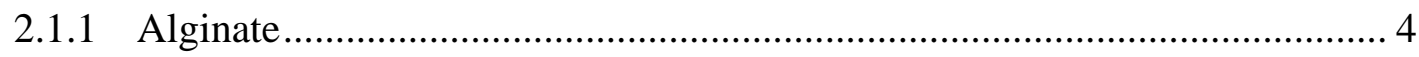

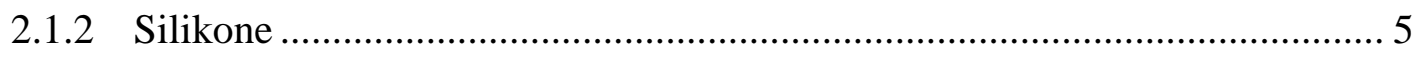

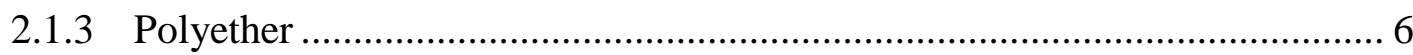

2.2 Überblick über die Abformtechniken ............................................................ 7

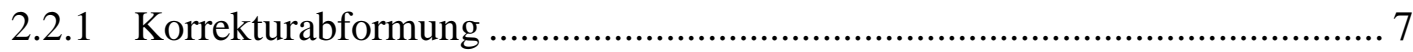

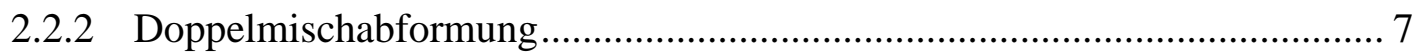

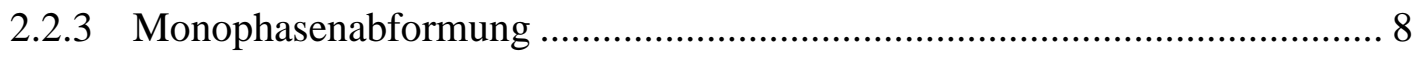

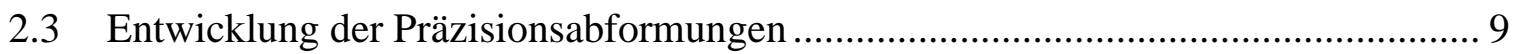

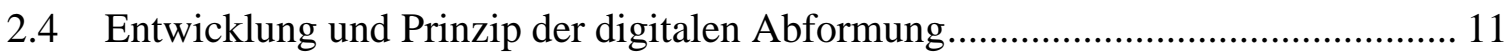

2.5 Erforderliche marginale Genauigkeit von Restaurationen .................................. 14

2.6 Möglichkeiten zur Bestimmung der marginalen Genauigkeit .............................. 15

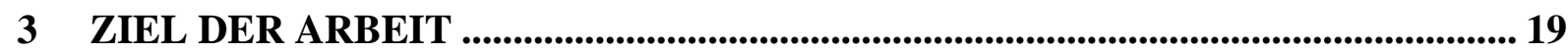

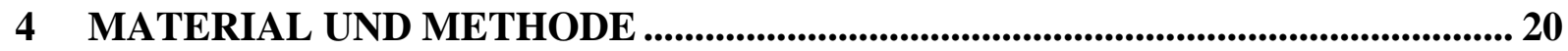

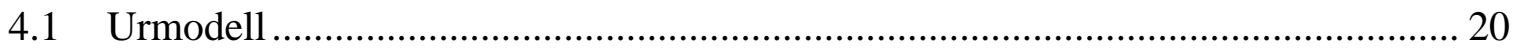

4.2 Durchführung der Abformungen und Herstellung der Prüfkörper ......................... 23

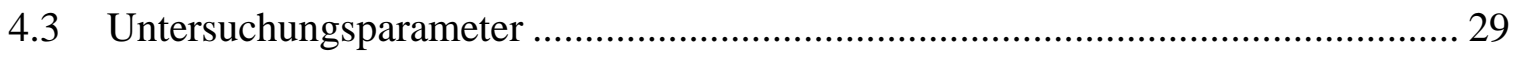

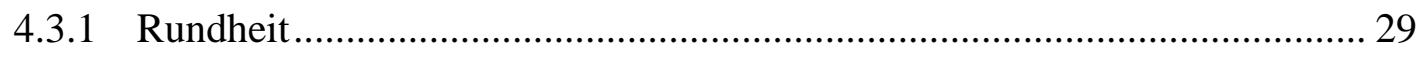

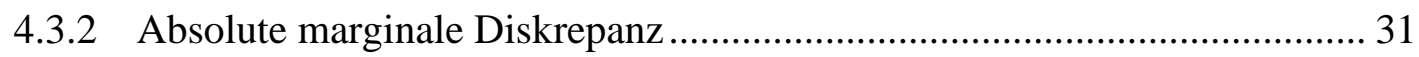




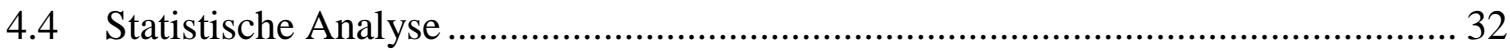

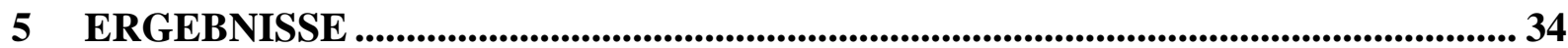

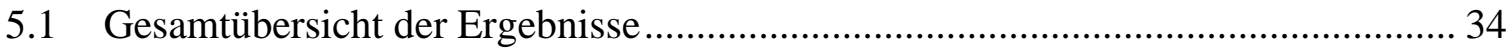

5.1.1 Rundheit: Bestimmung des Formfehlers ............................................. 34

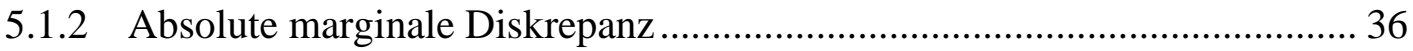

5.2 Ergebnisse des Vergleiches der angewendeten Abformverfahren ........................ 38

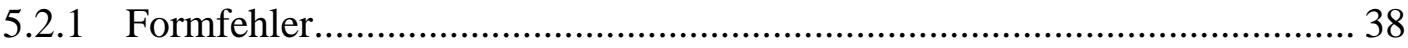

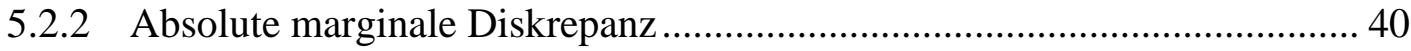

5.3 Ergebnisse des Vergleiches der verwendeten Materialien .................................... 43

5.4 Ergebnisse des Vergleiches der verschiedenen zahntechnischen Labore................ 45

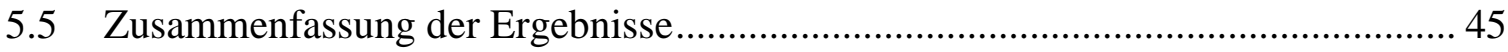

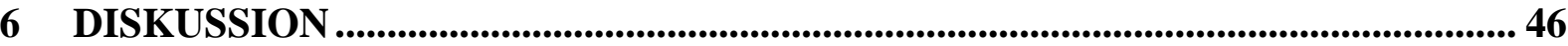

6.1 Diskussion der verwendeten Materialien und Methoden ...................................... 46

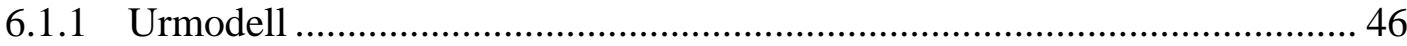

6.1.2 Durchführung der Abformungen und Herstellung der Prüfkörper .............. 47

6.1.3 Diskussion der Untersuchungsparameter.............................................. 49

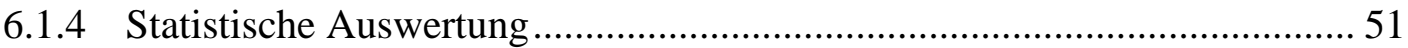

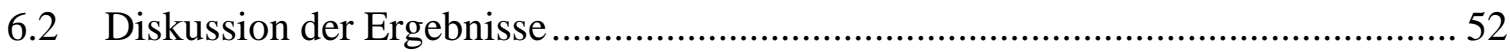

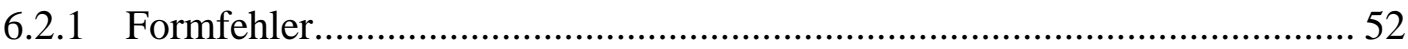

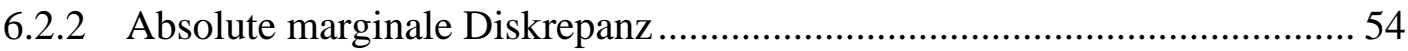

6.2.3 Diskussion der Ergebnisse der verwendeten Abformverfahren.................. 55

6.2.4 Diskussion der Ergebnisse des Vergleiches der verwendeten Materialien.. 57

6.2.5 Diskussion der Ergebnisse des Vergleiches der Zahntechnischen Laboratorien 


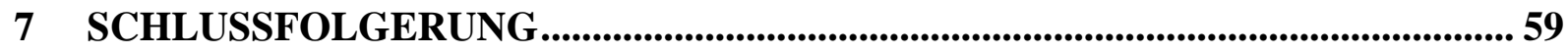

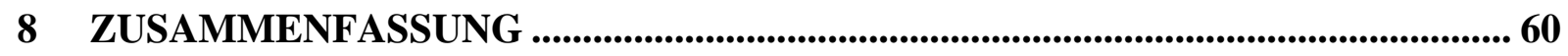

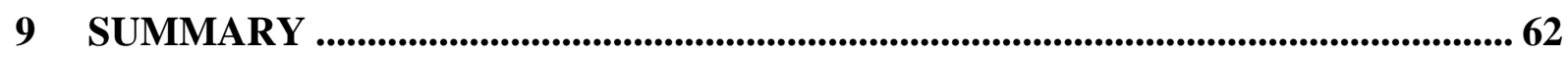

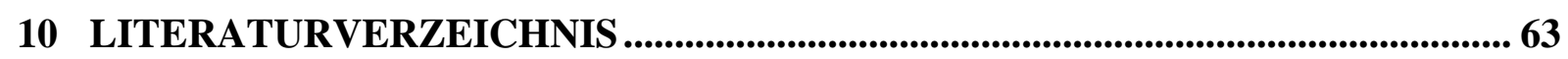

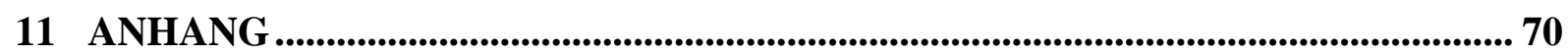

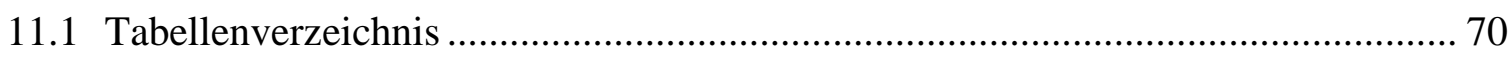

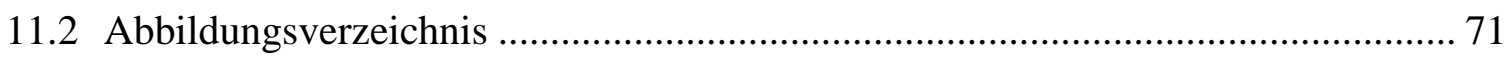

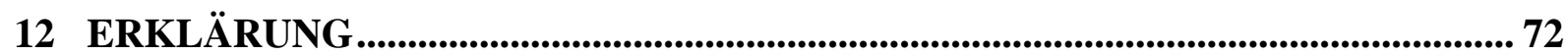

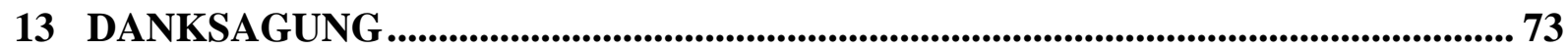




\section{Einleitung}

Bei der Herstellung von laborgefertigtem Zahnersatz spielt die Übertragung der Informationen aus der Mundhöhle des Patienten in das zahntechnische Labor eine entscheidende Rolle. Eine möglichst genaue Darstellung der anatomischen Strukturen ist notwendig, um eine qualitativ hochwertige, passgenaue Restauration zu erhalten. Je besser die Dimensionstreue und Detailwiedergabe des Abformmaterials, desto passgenauer ist auch der auf dieser Basis gefertigte Zahnersatz. Sind die Dimensionen präparierter Zähne nicht exakt wiedergegeben, kann der angefertigte Zahnersatz möglicherweise nicht in Sollposition gebracht werden. Gleichzeitig müssen Details im Kronenrandbereich möglichst genau dargestellt werden, um die langfristige Funktionstüchtigkeit einer Restauration zu gewährleisten. Klinische Studien belegen, dass die Qualität des Kronenrandes entscheidenden Einfluss auf den Erfolg oder Misserfolg einer festsitzenden Restauration hat, da zu große Randspalten über eine verstärkte Plaqueanlagerung zu Sekundärkaries und marginaler Parodontitis führen können ${ }^{4,26}$.

Die konventionelle Abformung mit Abformmaterialien wird in der Zahnmedizin seit mehr als 150 Jahren durchgeführt. In dieser Zeit unterlagen die verwendeten Materialien und Techniken einer stetigen Weiterentwicklung und Verbesserung, so dass dem Zahnarzt heute zahlreiche, gut funktionierende Abformsysteme zur Verfügung stehen. In der Praxis werden gegenwärtig zur Präzisionsabformung hauptsächlich Elastomere (A-Silikone, K-Silikone und Polyether) verwendet, da diese aufgrund ihrer Materialeigenschaften den Anforderungen am besten gerecht werden. In zahlreichen Studien wurde die Wiedergabegenauigkeit von Abformmaterialien bereits untersucht ${ }^{3,50}$.

Zu den gängigsten Abformtechniken zur Herstellung von festsitzendem Zahnersatz zählen die Doppelmischabformung und die Korrekturabformung. Die Vor- und Nachteile der jeweiligen Techniken zeigten sich bereits in zahlreichen Untersuchungen. Im Laborversuch wurden diese auch anhand der Größe des Randspaltes resultierender Kronen beurteilt ${ }^{79}$. Eine weitere Abformtechnik, mit der sich sehr genaue Abformungen erreichen lassen, ist die Monophasenabformung. Sie wird meist mit einem Polyether oder A- Silikon durchgeführt und es wird, aufgrund der höheren Fließfähigkeit des Materials, im Gegensatz zu den anderen Techniken meist ein individueller Abformlöffel benötigt ${ }^{82}$. 
Bislang galt die konventionelle Abformung mit Hilfe eines Abformmaterials als einzige Möglichkeit zur Herstellung eines Arbeitsmodells, auf dem laborgefertigter Zahnersatz hergestellt werden konnte. In den letzten Jahren gab es allerdings intensive Bemühungen, eine alternative Methode zu der mit zahlreichen, material- und methodenbedingten Fehlerquellen behafteten Arbeitskette der konventionellen Abformung zu finden. Oberstes Ziel war es hierbei, eine mindestens gleichwertige, detaillierte Darstellung aller relevanten intraoralen Bereiche zu erhalten. Dies versuchten verschiedene Entwickler mit intraoralen Scannern zu realisieren. Die digitale Abformung hat mittlerweile die Praxisreife erreicht und die ersten Geräte sind bereits in Praxen und an Kliniken in Betrieb. Gegenwärtig sind drei Systeme allgemein verfügbar: Das CEREC Connect (Sirona) System, der LCOS (Lava Chairside Oral Scanner) von 3M ESPE sowie der iTero Scanner (Cadent/ USA) welcher in Deutschland von der Firma Straumann vertrieben wird. Weitere Systeme, wie z.B. der cara TRIOS von Heraeus Kulzer werden aktuell in den Markt eingeführt. 


\section{Literaturübersicht}

Die Abformung zuvor präparierter Zahnhartsubstanz stellt in der Arbeitskette zur Herstellung von Zahnersatz einen zentralen Schritt dar. Je nach Art der vorliegenden Präparation und des später anzufertigenden Zahnersatzes kommen verschiedene Abformtechniken und Abformmaterialien zum Einsatz.

\section{1 Überblick über die gängigen Abformmaterialien}

Das generelle Prinzip der Abformung mittels eines Abformmaterials wurde im 18. Jahrhundert durch Pfaff in die Prothetik eingeführt. Er verwendete verflüssigtes Siegelwachs als Abformmasse. Durch Ausgießen des so gewonnenen Negativs mit Gips fertigte er ein Modell. 1820 wurde durch Delabarre der Abformlöffel als Träger für das Material erstmals beschrieben ${ }^{30}$.

Die meisten Abformmaterialien wurden allerdings in den letzten siebzig Jahren entwickelt. Eine zufriedenstellende Darstellung schwer abzuformender Unterschnitte gelang erst 1926 mit der Einführung der Hydrokolloide durch Poller ${ }^{61}$.

Die Weiterentwicklung führte zur Einführung von Zinkoxid-Eugenol Abformpasten 1933 und schließlich 1940 zum Alginat. Die Verwendung von Silikonen als Abformmaterial begann mit den kondensationsvernetzenden Silikonen in den 50er Jahren in Deutschland. Etwa zwanzig Jahre später kamen dann die additionsvernetzenden Silikone hinzu. Die Polyether wurden in den 60er Jahren eingeführt ${ }^{67,75}$.

An Abformmaterialien werden hohe Anforderungen, gerade im Bereich der Präzisionsabformung gestellt. Eine hohe Abformgenauigkeit ist von besonderem Interesse, das Material sollte zum Zeitpunkt der Abformung eine plastische Konsistenz aufweisen, feuchte-tolerabel (hydrophil) sein und möglichst gut an die abzuformenden Areale anfließen, um eine gute Detailwiedergabe zu erreichen. Beim Abbindevorgang sollten für eine ausreichende Dimensionsstabilität der Abformung möglichst keine, oder nur geringe Volumenveränderungen auftreten. 
Nach dem Abbinden sollte die Abformung ohne Beschädigung einfach aus dem Patientenmund zu entfernen sein. Natürlich wird auch eine Kompatibilität mit Modellwerkstoffen (z.B. Gips, Kunststoffe), eine gewisse Lagerstabilität und Desinfizierbarkeit gefordert. Üblicherweise werden Abformmaterialien in vier Hauptgruppen unterteilt ${ }^{82}$ :

- $\quad$ irreversibel starr $\rightarrow$

- reversibel starr $\rightarrow$

- irreversibel elastisch $\rightarrow$

- reversibel elastisch $\rightarrow$
Abformgipse; Zinkoxid- Eugenol- Pasten

Guttapercha; thermoplastische Kompositemassen

Alginat; Elastomere (Silikone, Polyether, Polysulfide)

Hydrokolloide

Nur ein kleinerer Teil dieser Materialien hat heute noch klinische Bedeutung. Insbesondere den irreversibel elastischen Abformmassen (Alginate, Elastomere) kommt gegenwärtig die größte Bedeutung zu, wobei Polysulfide in Deutschland keine Rolle spielen. Diese Materialien eignen sich bevorzugt zur Abformung präparierter Zahnhartsubstanz und zur Darstellung der umgebenden Weichgewebe ${ }^{80}$.

\subsubsection{Alginate}

Alginate werden heute besonders zur Situationsabformung verwendet. Sie werden in Pulverform geliefert und mit Wasser angemischt. Die Grundsubstanz der Alginate ist die Alginsäure, deren Salze wasserlöslich sind. Bei Wasserzugabe kommt es zu einer raschen Abbindereaktion zwischen der Alginsäure und Kalziumionen der zweiten Reaktionskomponente, dem Kalziumsulfat. Das Verhältnis von Pulver zu Wasser beim Anmischen differiert zwischen den Herstellern. Die Temperatur des verwendeten Wassers hat auf die Abbindezeit einen hohen Einfluss, $20^{\circ} \mathrm{C}$ sollten dabei nicht überschritten werden, da das Material sonst sehr schnell abbindet. Die Abformung sollte nach der Entnahme aus dem Patientenmund möglichst schnell weiter verarbeitet werden, da es während der Lagerung zu starken Dimensionsänderung durch 
Wasserverlust oder übermäßiges Quellen kommen kann. Eine kurze Lagerung (höchstens zwei Stunden) ist jedoch bei einer relativen Luftfeuchte von $100 \%$ möglich ${ }^{55}$.

Da das Rückstellvermögen, sowie die Dimensionstreue und Detailwiedergabe der Alginate im Gegensatz zu den Elastomeren wesentlich schlechter ist, werden sie zur Präzisionsabformung nicht eingesetzt ${ }^{59,82}$.

\subsubsection{Silikone}

Silikone, auch Polyvinylsiloxane genannt, können zur Durchführung zahlreicher Abformverfahren eingesetzt werden (Korrekturabformung, Sandwich- und Doppelmischtechnik, Einphasenabformung). Zwei verschiedene Silikone werden als Abformmaterialien genutzt. Dabei handelt es sich um kondensationsvernetzende (K-Silikone) und additionsvernetzende (ASilikone) Materialien. K-Silikone werden als Paste-Paste- bzw. Paste-Flüssigkeits-Systeme geliefert. Beim K-Silikon führt die nach Abschluss der Polykondensation unvermeidliche Verdunstung des Alkohols zu einer Schrumpfung des Materials, deren Ausmaß vom Füllstoffgehalt abhängig ist. Da die Füllkörper an der Reaktion nicht beteiligt sind, schrumpfen hochgefüllte Silikone weniger als niedriggefüllte. Die Schrumpfung von modernen KSilikonen lässt eine Lagerung der Abformung von 12 bis 24 Stunden zu, längere Lagerungszeiten sind nicht zu empfehlen ${ }^{85}$.

Bei den A-Silikonen kommt es während der Lagerung zu keiner weiteren Schrumpfung, da bei deren Abbindereaktion kein Kondensat entsteht. Sie können deshalb problemlos mehrere Tage gelagert werden. A-Silikone zeichnen sich gegenüber den K-Silikonen außerdem durch einen höheren E-Modul und einer höheren Zerreißfestigkeit aus. Aus diesen Gründen werden A-Silikone zur Präzisionsabformung gegenüber den K-Silikonen bevorzugt. Jedoch ist bei den A-Silikonen zu beachten, dass deren Abbindereaktion durch Latexpartikel, vor allem aus Schutzhandschuhen, und auch Metallsalzen, wie Aluminium- oder Eisenchlorid, die typischerweise in Adstringentien enthalten sind, inhibiert werden kann. Als Nachteil muss die große Hydrophobie der A-Silikone angesehen werden. Diese ist bedingt durch das Fehlen von polaren Anteilen in den Silikonmolekülen. Die Hersteller versuchen zunehmend, durch geeignete Zusätze diese Hydrophobie zu reduzieren ${ }^{81,84}$. 


\subsubsection{Polyether}

Polyether sind seit 1964 auf dem Markt erhältlich. Sie sind ausschließlich als Paste-PasteSysteme verfügbar. Die Basispaste besteht zum größten Teil aus einem Copolymerisat aus Ethylenoxid und Tetrahydrofuran. Der Vernetzungsmechanismus ist eine kationische Polyaddition. Da bei dieser Art von Vernetzungsreaktion keine flüchtigen Nebenprodukte abgespalten werden, treten bei einer Lagerung der Abformung nur sehr geringe Dimensionsänderungen auf. Die gegenüber den Silikonen lange Abbindezeit begünstigt außerdem einen größeren Abbau endogener Spannungen durch Fließvorgänge ${ }^{82}$.

Polyether werden meist mit individuellen Löffeln in einphasigen bzw. einzeitig-zweiphasigen Abformverfahren verarbeitet. Die Anwendung von Serienlöffeln ist zwar möglich, allerdings ist dies aufgrund der geringen Standfestigkeit des Materials nicht zu empfehlen. Polyether eignen sich nicht zur Durchführung des Korrekturverfahrens, da sie nur sehr schwer beschneidbar sind und sich die beiden Phasen nicht miteinander verbinden ${ }^{81}$.

Teilweise wurde in der Literatur bereits das Auftreten allergischer Reaktionen im Zusammenhang mit Polyethern beschrieben. Die Katalysatorkomponente wurde dafür meist in erster Linie verantwortlich gemacht. Bei anamnestisch auffälligen Patienten sollte daher auf ein anderes Material ausgewichen werden ${ }^{24,46}$. 


\section{2 Überblick über die Abformtechniken}

Das Ergebnis einer Abformung mit herkömmlichen Mitteln hängt nicht nur von den Eigenschaften der verwendeten Abformmaterialien ab, sondern ist auch mit der Eignung der gewählten Abformtechnik für die jeweilige klinische Situation verbunden. Die Abformtechniken unterscheiden sich sowohl in der Anzahl der verwendeten Abformmassen (einphasig bzw. zweiphasig) wie auch in ihren zeitlichen Arbeitsschritten (einzeitig bzw. zweizeitig).

\subsubsection{Korrekturabformung}

Die Korrekturabformung stellt eine der momentan meist verwendeten Abformtechniken dar. Zur Durchführung der Korrekturtechnik wird im ersten Arbeitschritt eine Vorabformung mit einem knetbaren Material genommen, welches nach dem Aushärten ausgeschnitten wird und im zweiten Schritt mit einem niedrigviskösen Material „korrigiert“ wird (zweizeitigzweiphasige Abformung). Dabei kommt es zu einer Dimensionsverkleinerung des wiedergegebenen Stumpfes durch nicht vollständige elastische Rückstellungen der Abformung nach Stauchung des Vorabformungsmaterials in der Korrekturphase ${ }^{9,}{ }^{49}$. Nach der Entnahme aus dem Mund stellt sich die Masse der Erstabformung zurück und die Lumina werden dadurch verzerrt. Insgesamt lassen sich mit der Korrekturtechnik bei sachgemäßer Anwendung gute Ergebnisse erzielen. Sollen subgingival gelegene Präparationen dargestellt werden, so ist dies mit der Korrekturabformung am besten zu erzielen, da das Korrekturmaterial durch den Staudruck bei Einbringen in den Sulkus ,gepresst“" wird ${ }^{82}$.

\subsubsection{Doppelmischabformung}

Eine weitere Methode zur Abformung ist die einzeitig-zweiphasige Doppelmischtechnik, diese wird in einem Arbeitschritt vorgenommen. Es werden zwei Materialien unterschiedlicher Viskositäten (hochvisköses Löffelmaterial und niedrigvisköses Material) gleichzeitig verwendet. Nachdem der abzuformende Zahn im Mund direkt mit dem dünnfließenden Material umspritzt wird, schiebt man den mit dem zähplastischen Material beschickten Löffel unmittelbar 
nach. Beide Phasen vermischen sich und binden ab ${ }^{45}$. Die Wiedergabegenauigkeit ist gut, es kann jedoch kein Staudruck erzielt werden, um subgingivale Bereiche darzustellen ${ }^{50}$.

Eine der Doppelmischtechnik ähnliche Abformmethode ist die Sandwichabformung. Im Unterschied zur Doppelmischabformung wird das dünnfließende Material nicht direkt um die präparierten Zähne im Mund, sondern als zweite Schicht auf das Löffelmaterial appliziert ${ }^{82}$.

\subsubsection{Monophasenabformung}

Die Monophasenabformung, eine einzeitig-einphasige Technik, stellt eine ebenfalls häufig verwendete Abformmethode dar. Die Konsistenz des verwendeten Materials wird so gewählt, dass es gerade noch mittels einer Abformspritze verspritzt werden kann. Das Material wird dabei einerseits mit Hilfe einer Spritze direkt im Mund des Patienten appliziert und andererseits zum Befüllen des Abformlöffels verwendet. Mit dieser Methode sind sehr genaue Abformungen möglich. Jedoch kann, wie auch bei der Doppelmisch- und Sandwichtechnik im Gegensatz zur Korrekturtechnik kein wesentlicher Staudruck erzielt werden. Daher ist die Verwendung eines individuellen Löffels unbedingt zu empfehlen. ${ }^{53,82}$ 


\subsection{Entwicklung der Präzisionsabformungen}

Um ein detailgetreues Modell und einen gut passenden Zahnersatz zu erhalten, waren die Bemühungen schon in der Vergangenheit groß, eine möglichst optimale Technik in Verbindung mit einem geeigneten Abformmaterial zu finden. Die Bemühungen, eine alternative Technik zur vorherrschenden Kupferringabformung zu finden, mit der auch subgingivale Bereiche gut dargestellt werden konnten, waren erheblich. 1937 führte Sears Versuche mit der sogenannte Kerr-Permlastik-Gesamtabformung als Doppelmisch- und als Korrekturverfahren durch, für die er zunächst Hydrokolloide und anschließend Polisulfide verwendete ${ }^{9}$. 1956 beschrieb Stahl unter der Bezeichnung „Doppelabdruckverfahren“ erstmals die Korrekturtechnik. Er verwendete zur Vorabformung jedoch noch eine thermoplastische Kompositmasse und zur Korrekturphase eine elastische Kautschuk-Silizium Masse ${ }^{69} .1962$ verwendete Böttger auch für die Vorabformung ein elastisches Material ${ }^{8} .1965$ wurde das Verfahren von Hofmann weiterentwickelt ${ }^{31}$. Als Material für die erste Phase verwendete Hofmann nicht wie Stahl ein thermoplastisches Material, sondern ein Silikon. Die Korrekturphase wurde, wie auch schon durch Stahl, mit einem dünnfließenden Silikon durchgeführt. Auch die Probleme der Dimensionsverkleinerung des resultierenden Stumpfes wurden durch Hofmann bereits beschrieben. Hofmann führte die Korrekturphase noch ohne vorhergehendes Ausschneiden der Vorabformung durch. Dies wurde dann 1966 durch Knoblauch beschrieben ${ }^{43}$. Weitere Studien dazu wurden unter anderem 1978 von Lehmann und Burgdorf durchgeführt ${ }^{49}$, die zu dem Ergebnis kamen, dass durch das Anbringen von Abflussrinnen in der Vorabformung eine dimensionsgetreuere Wiedergabe erzielt werden konnte. 1990 verglichen Wichmann, Borchers und Limmroth die gängigsten Abformmethoden (Monophasen-, Doppelmisch- und Korrekturabformung) mit verschiedenen Materialien ${ }^{78}$. Sie kamen zu dem Ergebnis, dass die Modellstümpfe, die aus den Korrekturabformungen resultierten, kleiner als der Originalstumpf und konkav verzerrt waren. Als Erklärung dafür gaben sie die elastische Deformation des Materials während der Korrekturphase an. Wöstmann, Hönig und Ferger verglichen 1998 hand- und maschinenangemischte Abformmaterialien und kamen auch zu dem Ergebnis, dass die Stümpfe der auf Korrekturabformungen basierenden Modelle kleiner waren als das Original. Sie erklärten sich dies durch erhöhte Verdrängungseffekte auf Grund der geringen ShoreA Härte des untersuchten A-Silikons ${ }^{83}$. Sehr gute Ergebnisse im Bereich der Detailwiedergabe erhielten sie bei Durchführung der Doppelmischtechnik. In vielen Untersuchungen zeigte sich, dass bei Anwendung der Doppelmisch- im Gegensatz zur Korrekturabformung die 
Stumpfdimensionen exakter wiedergegeben wurden ${ }^{50}$. In den darauf folgenden Jahren untersuchten verschieden Autoren die Korrekturtechnik und versuchten eine Lösung für das Problem der verkleinerten Wiedergabe der Stumpfform zu finden. Mehrfach versuchten diese über Veränderungen der Präparationswinkel oder der Schichtstärken des Korrekturmaterials eine geringere Dimensionsabweichung zu erreichen ${ }^{58,66}$. Fenske et al. rieten in ihrer Studie 2001 dazu, dass Meistermodell nach Korrekturabformung hohl zu legen und somit den verkleinerten Stumpf auszugleichen ${ }^{20}$. Bis heute konnte dieses Problem während der Durchführung der Abformung nicht gelöst werden. Ein Ausgleich der Dimensionsungenauigkeit kann jedoch in der weiteren Werkstoffkette der Herstellung des Zahnersatzes im Zahntechnischen Labor weitestgehend hergestellt werden.

Eine ebenfalls in der zahnärztlichen Praxis weit verbreitete Abformmethode ist die Monophasenabformung. Sowohl A-Silikone, als auch Polyether werden zu deren Durchführung verwendet. Die Monophasenabformung zeichnet sich besonders durch eine hohe Wiedergabegenauigkeit aus, was bereits mehrere Autoren in ihren Untersuchungen belegten. Dabei kommt es im Gegensatz zu der bei der Korrekturtechnik gemessenen Stumpfverkleinerung zu einer, im Vergleich allerdings wesentlich geringeren, Vergrößerung der resultierenden Modellstümpfe $21,78,79$.

Besonders im Bereich der Implantatprothetik ist eine möglichst exakte, dreidimensionale Übertragung der Implantatpositionen aus dem Mund des Patienten auf das zur Herstellung des Zahnersatzes notwendige Arbeitsmodell besonders wichtig. Bereits geringe Abweichungen können dazu führen, dass die angefertigte Restauration am Patienten später nicht in Sollposition gebracht werden kann. Aufgrund ihrer hohen Dimensionstreue und guten werkstoffkundlichen Eigenschaften werden besonders Polyether in diesem Bereich zur Abformung verwen$\operatorname{det}^{29}$. 


\subsection{Entwicklung und Prinzip der digitalen Abformung}

An der Entwicklung von Verfahren zur Herstellung von Zahnersatz auf der Basis intraoraler Abformungen wurde bereits zu Beginn der 80er Jahre gearbeitet. Mörmann und Brandestini entwickelten ab 1980 an der Universität Zürich das CEREC-System, eine Abkürzung für CEramic REConstrution. 1986 brachte die Firma Sirona (damals Siemens) das CEREC-System auf den Markt. Dieses wurde kontinuierlich weiterentwickelt und ist mittlerweile in der 4. Generation erhältlich. Ziel der Entwicklung war es, auf der Basis einer optischen Abformung vollkeramische Restaurationen innerhalb einer Behandlungssitzung direkt herzustellen. Dazu wurde eine CNC Fräseinheit zu dem Scansystem kombiniert, mit der aus vorgefertigten Keramikblöcken erste zahntechnische Arbeiten hergestellt werden konnten. Zunächst war es mit dem CEREC 1 nur möglich Inlays zu fertigen. Das erste chairside-Inlay wurde 1985 hergestellt. Mit CEREC 2 konnten dann erstmals auch vollkeramische Kronen gefräst werden. 2000 folgte die Softwareversion 3 und 2003 die Weiterentwicklung in 3D. Nun war es für den Anwender möglich die Restauration am Computer vor dem Fräsvorgang noch vielfältiger zu bearbeiten ${ }^{16,57}$. Die aktuelle CEREC-Bluecam arbeitet, wie auch schon ihre Vorgänger, nach dem Prinzip der Streifenlichtprojektion. Das Objekt wird dabei aus einer Richtung beleuchtet und aus einer anderen beobachtet, dazwischen spannt sich der sogenannte Triangulationswinkel auf und es werden sogenannte „Höhenprofile“ wiedergegeben ${ }^{28}$. Der Scanvorgang erfolgt als Aufnahme vieler Einzelbilder. Die Kamera wird in einem definierten Abstand über der Zahnreihe geführt und das System löst in einem verwackelungsfreien Moment selbstständig eine Bildaufnahme aus. Vor Durchführung des Scans muss der jeweilige darzustellende Bereich zur Vermeidung von Reflexionen bepudert werden ${ }^{35}$. Bei der CEREC-Bluecam kommt blaues Licht mit einer Wellenlänge von 470nm, anstelle des bislang verwendeten Infrarotlichtes mit einer Wellenlänge von ca. 820nm zum Einsatz, wodurch die Genauigkeit der Messungen und die Auflösung um etwa 60\% stiegen. Die veränderte Linsenanordnung des digitalen Kamerasystems sorgte für weniger notwendige, automatische Filterung und dadurch für bessere Detailwiedergabe. Das sogenannte „Rauschen“ (zufällig auftretende Messfehler) wurde ebenfalls geringer, was dazu führt, dass weniger Daten automatisch durch die Software herausgefiltert werden mussten ${ }^{56}$. Mehl führte 2009 eine Vergleichsstudie zwischen der neuen CEREC-Bluecam und der CEREC-3D-Kamera durch. Er verglich die Scans mit Hilfe einer Übertragungssoftware. Die Genauigkeit der CEREC-Bluecam war signifikant besser ${ }^{56}$. 
2008/ 2009 kam mit dem LCOS (Lava Chairside Oral Scanner; 3M Espe, Seefeld) ein weiterer intraoraler Scanner auf den Markt. Das Funktionsprinzip wurde 2006 am Massachusetts Institute of Technology (MIT) entwickelt. Der Aufnahmekopf des LCOS besteht ebenfalls aus einem digitalen Kamerasystem, welches 192 LEDs, 22 Linsen und 3 CCD-Sensoren umfasst. Die Scans werden nach dem Verfahren des sogenannten „Active Wavefront Sampling“ als 3D-Videoaufnahme angefertigt. Die Erfassung der Zahnform erfolgt hierbei durch die Bewegung der Kamera über die Zähne. Durch Positionsänderung der einzelnen Bildpunkte während der Videoaufnahme kann so deren Abstand zur Kamera ermittelt werden, wodurch insgesamt ein dreidimensionales Bild entsteht ${ }^{23,33}$.

Der iTero Scanner der Firma Straumann wurde vor wenigen Jahren auf den Markt gebracht und arbeitet nach dem Prinzip der konfokalen Bildgebung. Die Bildebene wandert dabei während der Aufnahme in der Vertikalen auf und ab und nur der aktuell scharfe Bildbereich wird vom System erfasst und berechnet ${ }^{37,39}$.

Weitere Systeme werden in näherer Zukunft wahrscheinlich folgen (z.B. cara Trios von Heraeus Kulzer).

Bei der klinische Anwendung des LCOS, wie auch bei dem CEREC-Scanner, ist es notwendig vor dem Scanvorgang die Zahnoberflächen zur Schaffung einer kontrastreichen Oberfläche mit einem speziellen Puder, welches überwiegend aus Titandioxid besteht, zu bestäuben. Alle Arbeitsschritte müssen unter Trockenlegung durchgeführt werden. Während die Zähne beim CEREC-System vollständig bedeckt sein müssen, ist es für den LCOS ausreichend, eine dünne, nicht deckende Puderschicht zu applizieren. Beim iTero Scanner kann sogar vollständig darauf verzichtet werden ${ }^{33}, 35,39$. Zur Darstellung und Erfassung infragingival gelegene Präparationsgrenzen muss allerdings häufig mit Retraktionsfäden gearbeitet werden. Hierbei gilt folgendes Prinzip: Ist die Präparationsgrenze mit bloßem Auge vollständig erkennbar, kann sie auch von den Kamera-Systemen erfasst werden.

Eine der ersten Untersuchungen zur Genauigkeit von auf optischen Abformungen basierendem Zahnersatz wurde 2004 von Reich durchgeführt. Er fertigte Restaurationen auf der Basis von intra- und extraoralen Scans mit CEREC 2 und untersuchte diese nach 3 Jahren. Das Hauptkriterium war hier allerdings nicht die Untersuchung der Scanverfahren sondern die Beständigkeit großer CAD/CAM-gefertigter Restaurationen ${ }^{63}$. Bereits in den 90er Jahren gab es Veröffentlichungen zum Thema CAD/CAM-gefertigter Restaurationen. So untersuchte unter anderem Bindl 1999 die marginale Passung von mit CEREC 2 gefrästen Frontzahnkronen ${ }^{6} .2005$ verglichen Luthard et al. die optische Abformung mit CEREC 3D und die extra- 
orale Digitalisierung. Die Datensätze wurden mit Hilfe einer entsprechenden Software verglichen und die Genauigkeit bestimmt. Bei dieser Untersuchung war die extraorale Digitalisierung die genauere Methode ${ }^{51}$. In den nächsten Jahren folgten weitere zahlreiche Untersuchungen vor allem im Bereich der Genauigkeiten von CAD/CAM-gefertigtem Zahnersatz. So fand Kokubo 2005 Randspalten von $66 \mu \mathrm{m}$ bei CAD/CAM-Vollkeramikkronen ${ }^{44}$. Tsitrou untersuchte 2007 mit CEREC 3D gescannte Kunststoffkronen und erhielt Ergebnisse bei der Messung der marginalen Genauigkeit von 77-105 $\mu \mathrm{m}^{74}$. Akbar et al. verglichen 2006 die marginale Passung von Kunststoffkronen basierend auf intraoralen Scans mit CEREC 3D an extrahierten Zähnen mit 2 unterschiedlichen Präparationsformen. Im Mittel wurden bei einer starken Streuung Genauigkeiten von $100 \mu \mathrm{m}$ erreicht und es konnte kein Einfluss der Form der Präparationsgrenze festgestellt werden ${ }^{1} .2009$ untersuchte D`Arcy die Passgenauigkeit von auf intraoralen CEREC 3D-Scans basierenden Keramikkronen verglichen mit auf konventionellen Korrekturabformungen basierenden Kronen. Es bestanden keine signifikanten Unterschiede im Bereich der Messung der Randspalten der Kronen in dieser Studie ${ }^{10} .2010$ und 2011 erschienen weitere Publikationen zum Thema digitale Abformung. Die meisten Studien beschäftigten sich mit dem CEREC-System. So untersuchten zum Beispiel Fasbinder et al. mehrfach die Genauigkeit von auf CEREC 3D-Scans basierenden Kronen ${ }^{17}{ }^{18}$. Da Costa scannte Onlaypräparationen mit CEREC 3D intra- und extraoral mit und ohne Scanpuder am Phantomkopf. Die Ergebnisse für die Randspaltbreiten lagen dabei zwischen 111,6 und $161,4 \mu \mathrm{m}$ ohne signifikante Unterschiede zwischen den Techniken ${ }^{11}$. Syrek et al. verglichen schließlich 2010 in einer klinischen Studie erstmals Kronen, hergestellt auf Basis von intraoralen Scans mit dem LCOS, mit auf Korrekturabformungen basierenden Kronen. Bei dieser Untersuchung wurde jeweils der gleiche Zahn mit beiden Techniken abgeformt. Die auf den intraoralen Scans basierenden Kronen waren den konventionell hergestellten in ihrer marginalen Genauigkeit überlegen $(49 \mu \mathrm{m}-71 \mu \mathrm{m}){ }^{72}$. Ender und Mehl verglichen 2011 in einer Laborstudie konventionellen Abformungen mit der CEREC-Bluecam und dem LCOS. Die Wiedergabegenauigkeit testeten sie anhand der Abweichungen der resultierenden Modelle von dem zuvor abgeformten Urmodell und die Präzision der Methoden durch den Vergleich der Modelle untereinander. Die Ergebnisse zeigten, dass die digitalen Verfahren absolut mit den konventionellen Techniken vergleichbar waren ${ }^{15}$. 


\subsection{Erforderliche marginale Genauigkeit von Restaurationen}

Zum Thema marginale Genauigkeit wurden bislang zahlreiche Untersuchungen, sowohl in vitro als auch in vivo durchgeführt, um eine Aussage darüber treffen zu können, in welcher Größenordnung ein Randspalt einer Restauration als akzeptabel angesehen werden kann.

Bei reinen Laboruntersuchungen wurden meist Passgenauigkeiten von unter 100 $\mu \mathrm{m}$ erreicht 70, 73 . Die Forderungen an die erreichbare Randschlussgenauigkeit lagen in der Vergangenheit

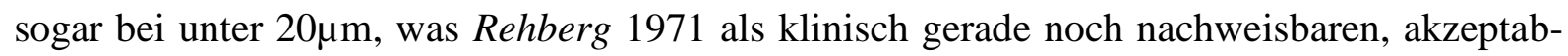
len Randspalt bezeichnete. Von einem Randschluss könne man nur sprechen, wenn kein Übergang zwischen Zahn und Krone sicht- oder tastbar sei ${ }^{62}$. Dreyer-Jörgensen forderte 1958 eine marginale Diskrepanz von höchstens $50 \mu \mathrm{m}{ }^{13}$. Laut einer Stellungnahme der DGZMK im Jahr 1999 sollte der Randspalt möglichst nicht mehr als $100 \mu \mathrm{m}$ betragen ${ }^{77}$. Andere Autoren bezeichnen Werte von bis zu $200 \mu \mathrm{m}$ als akzeptabel ${ }^{52}$.

Bei in vivo- Untersuchungen war es deutlich schwerer als im Laborversuch, eine reproduzierbare Aussage über die Dimension des vorhandenen Randspaltes zu treffen. Da die zu untersuchenden Zähne mit den jeweiligen Restaurationen sich ja noch im Mund des Patienten befanden, mussten andere Messmethoden herangezogen werden, als sie im Labor zur Verfügung standen. Schließlich wurden, um diese Problematiken zu umgehen, Zähne die zur Extraktion vorgesehen waren mit Zahnersatz versorgt und nach Extraktion im Labor untersucht ${ }^{5,68}$.

Beschnidt et al. untersuchten fünf verschiedene Vollkeramikkronen vor und nach zyklischer

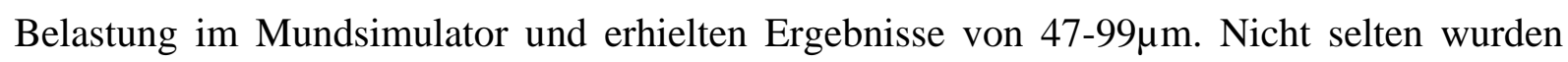
allerdings Größenordnungen von über $200 \mu \mathrm{m}$ erreicht, wie beispielsweise in einer Studie von Spiekermann 1986, wo die mittleren Messwerte sogar bei $382 \mu$ m lagen ${ }^{68}$.

Auch Wöstmann, Dohle und Rettberg führten eine Untersuchung mit zur Extraktion vorgesehenen Zähnen durch, die sie zunächst präparierten, abformten und dann extrahierten. Die gemessenen marginalen Diskrepanzen der angefertigten Restaurationen reichten von unter $200 \mu \mathrm{m}$ bis $\mathrm{zu} 680 \mu \mathrm{m}^{79}$.

Die geforderten maximalen marginalen Randspalten waren, wie die erwähnten Studien belegen, klinisch schwer erreichbar. Im Allgemeinen wurden als Ursache für die Diskrepanzen zwischen den in vitro und in vivo ermittelten marginalen Genauigkeiten klinische Faktoren, wie zum Beispiel subgingival gelegene Präparationsgrenzen, Speichelfluss oder Sulkusblutungen vermutet ${ }^{79}$. 
Der Einfluss auf die parodontale Gesundheit wurde unter anderem von Grasso et al. bereits 1985 untersucht. Sie stellten fest, dass Plaqueanlagerung, sowie das Auftreten von Gingivitis und Parodontitis bei Restaurationen mit inadäquatem Randspalt vermehrt auftraten ${ }^{26}$. Auch Bader et al. führten 1991 eine Studie zu diesem Thema durch. An subgingival gelegenen Kronenrändern zeigten sich bereits signifikant erhöhte Entzündungszeichen. Generell stellten sie fest, dass Zähne die mit Kronen versorgt waren, eher zu marginalen Parodontiden neigten als unversorgte Zähne. Die untersuchten Patienten waren in ein regelmäßiges RecallProgramm mit professioneller Zahnreinigung eingebunden, was zu einer Reduzierung der Problematik führte ${ }^{4}$.

\subsection{Möglichkeiten zur Bestimmung der marginalen Genauigkeit}

Bei der Beurteilung der Randspalten von festsitzendem Zahnersatz kamen und kommen immer noch vor allem folgende Verfahren zur Anwendung, die eine reproduzierbare Aussage ermöglichen sollen:

- klinische Inspektion (visuell und taktil)

- röntgenologische Untersuchung

- Herstellung eines Replikas

- indirekte Messung mittels eines Silikonfilmes

Bei der klinischen Inspektion des Kronenrandbereiches kann dieser zunächst visuell und mit Hilfe der gewöhnlichen zahnärztlichen Sonde beurteilt werden. Mit dieser Methode können laut Rehberg Diskrepanzen bis zu einer Grenze von $20 \mu \mathrm{m}$ ermittelt werden, da diese gerade noch ertastet werden könnten. Eine genauere Einteilung sei allerdings nicht möglich ${ }^{62}$. 
Marxkors entwickelte in Zusammenarbeit mit der Firma Aesculap spezielle Messsonden, deren Ende einer Hakensonde ähnelte. Die Spitzendurchmesser waren in $100 \mu \mathrm{m}$ Schritte eingeteilt. Der größte Spitzendurchmesser betrug 0,5mm, was als grobe Ungenauigkeit anzusehen war. Besonders in den Approximalräumen war deren Anwendung jedoch schwierig. Zusätzlich war das Ergebnis auch noch von den taktilen Fähigkeiten des jeweiligen Untersuchers abhängig ${ }^{25,62}$.

Um die schwer zugänglichen Approximalräume besser beurteilen zu können, stellte die röntgenologische Untersuchung eine ergänzende Methode dar. Es war jedoch mit dieser Methode nicht möglich die übrigen Bereiche des Kronenrandes zu beurteilen. Weiterhin konnte es schon durch geringe Abweichungen vom orthoradialen Strahlengang zu Verzerrungen kommen, die die Aufnahmen unbrauchbar machen konnten ${ }^{19}$.

Die marginale Diskrepanz ließ sich außerdem anhand der Herstellung eines Replikas des marginalen Bereiches bestimmen. Dazu wurde eine Abformung über die jeweilige eingesetzte Restauration genommen. Diese wurde dann entweder mit Gips ausgegossen oder direkt beurteilt $^{25}$.

Die bereits vielfach beschriebene Anfertigung eines indirekten Silkonfilmes stellt eine weitere Technik dar. Sowohl bei in vitro, als auch in vivo Untersuchungen wurde diese Methode bereits angewandt. Die zu vermessenden Kronen wurden dazu mit einem dünfließenden Silikon gefüllt, auf dem zugehörigen Stumpf in Sollposition gebracht und nach dem Aushärten des Silikons wieder entfernt. Zur Stabilisierung des Silikonfilmes wurde dieser häufig mit einem Heavybody- oder Putty-Material, welches nach der Entnahme in das Lumen appliziert wurde, stabilisiert. Anschließend wurde der entstandene Silikonfilm im Bereich der Präparationsgrenze zumeist unter einem Mikro- oder Makroskop im Labor vermessen ${ }^{7,73}$. Laurent et al. ermittelten mit dieser Methode marginale Genauigkeiten von $54-90 \mu \mathrm{m}{ }^{47}$. DÀrcy et al. erhoben Messwerte von $174 \pm 48 \mu \mathrm{m}^{10}$. 
In vielen Studien wird auf eine genaue Angabe der Messstrecke verzichtet, die zur Bestimmung der marginalen Diskrepanz dient, was die Vergleichbarkeit erschwert. Bei der Definition der zur Bestimmung des Randspaltes zu vermessenden Strecke gab es in der Literatur verschiedene Ansätze. Donath und Roth formulierten dazu 1987 die folgenden Kriterien ${ }^{12}$ :

- Passgenauigkeit: Die Distanz von der Präparationsgrenze zur Innenfläche des Kronenrandes;

- Zementspaltbreite: Strecke von der präparierten Zahnoberfläche zur Innenfläche der Krone im Winkel von $90^{\circ}$;

- vertikaler Randschluss: Abstand von der Präparationsgrenze zum Kronenrand;

- Stufenbildung: Strecke von der Zahnoberfläche zum Kronenrand im rechten Winkel.

In der folgenden Abbildung1 sind die Definitionen noch einmal zur Verdeutlichung dargestellt.
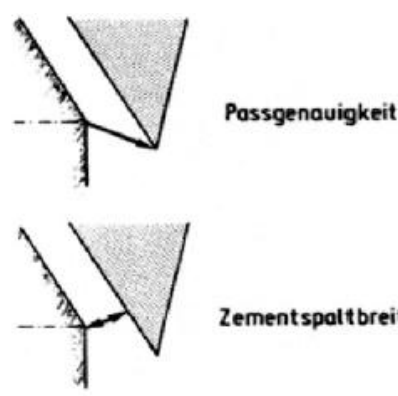

Zementspaltbreite

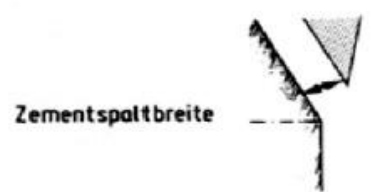

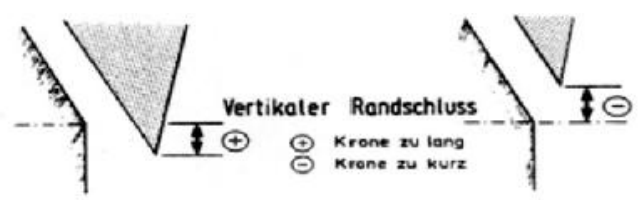

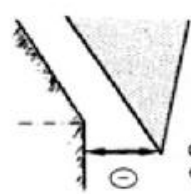

Stufenbildung

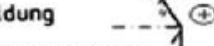

(†) boberhalb der Proparationsgrenze

Abb.1: Definition des Randschlusses nach Donath und Roth ${ }^{12}$.

Die 1989 von Holmes formulierte Nomenklatur wurde bereits in zahlreichen Untersuchungen zur Beurteilung der marginalen Bereiche von festsitzendem Zahnersatz verwen$\operatorname{det}^{41,44,74,79}$.

Die Einteilung erfolgte in verschiedene geometrische Strecken (Abb.2): ,internal gap“; „,marginal gap“; ,overextended margin“; ,underextended margin“; ,,vertical marginal discrepancy”; ,horizontal marginal discrepancy”; ,,absolute marginal discrepancy"; ,seating discrepancy"; 

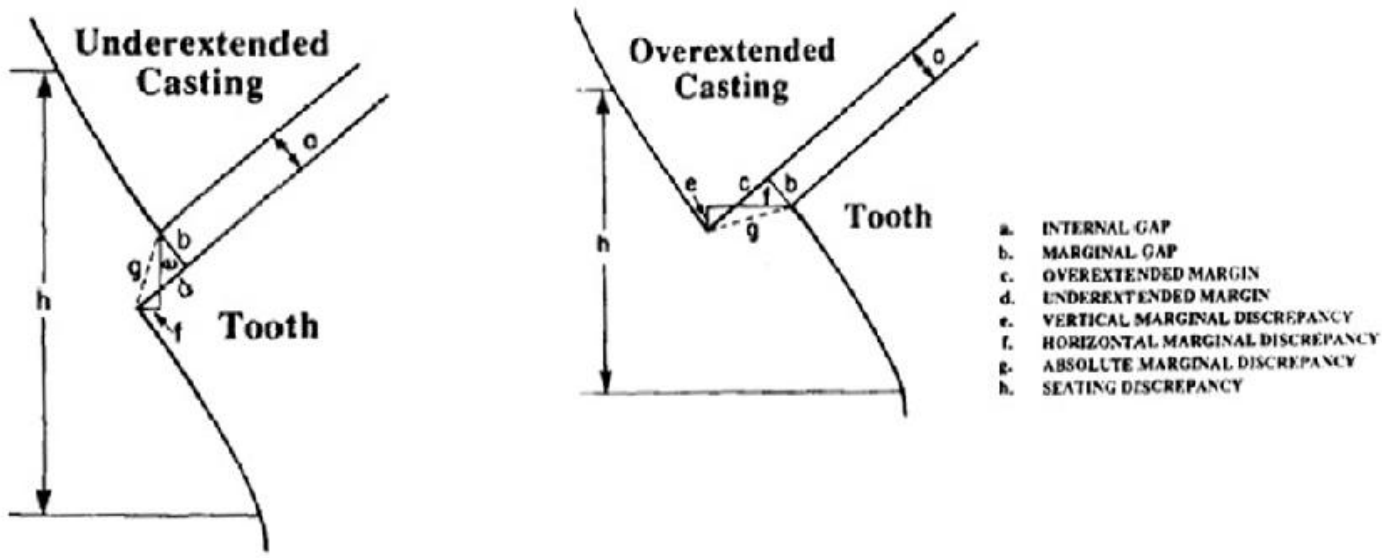

Abb.2: Absolute marginale Diskrepanz nach Holmes et al. ${ }^{32}$.

Es ergeben sich daraus die in Abbildung 3 dargestellten Möglichkeiten für den Randschluss.
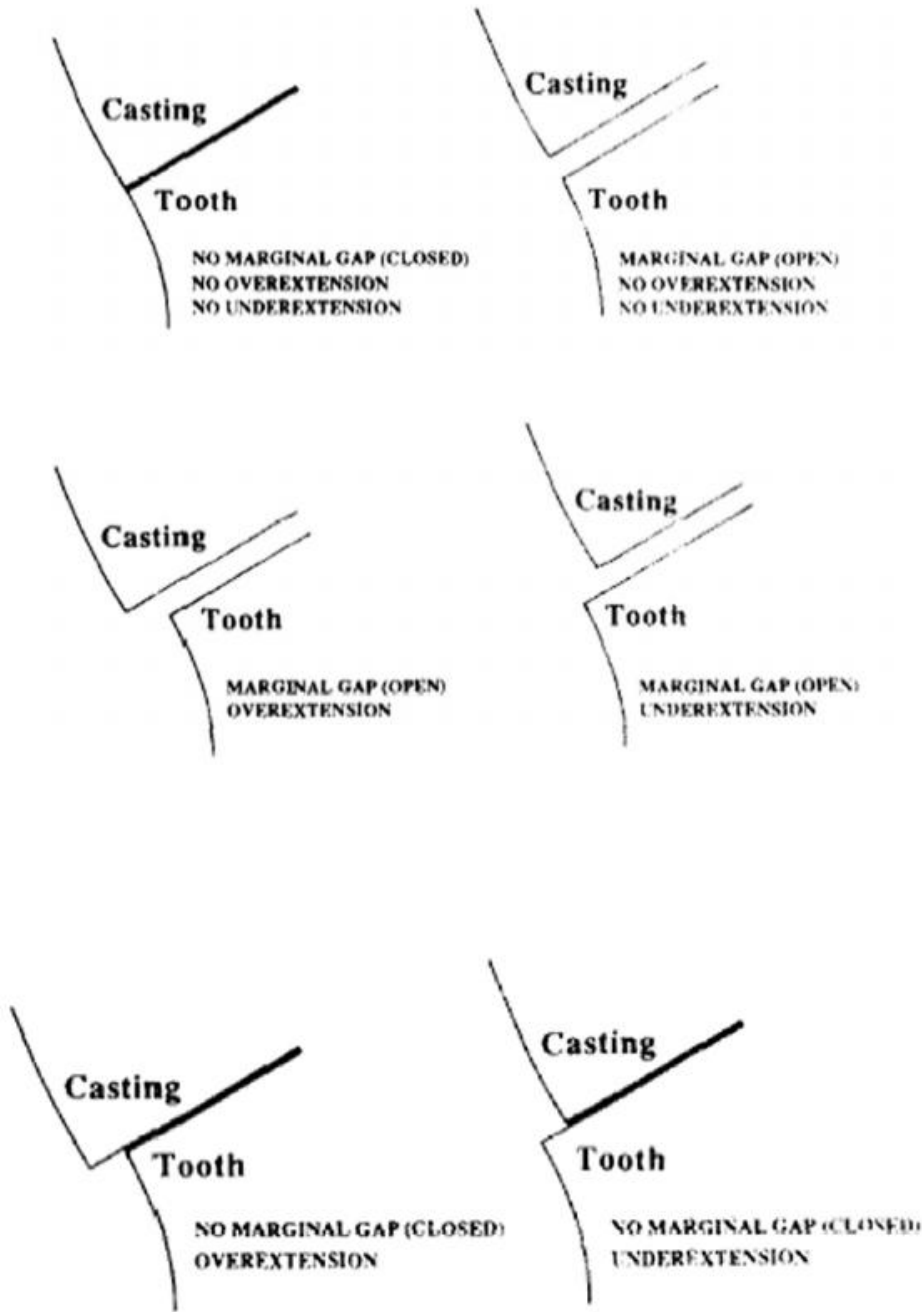

Abb.3: Formen des Randschlusses nach Holmes et al. ${ }^{32}$. 


\section{Ziel der Arbeit}

Das Ziel dieser in vitro Studie war es, die Passgenauigkeit von Kronen, die einerseits auf der Basis klassischer Abformtechniken hergestellt und andererseits via intraoraler Scannverfahren übertragen und gefertigt wurden, zu vergleichen. Zusätzlich sollte der Einfluss unterschiedlicher verwendeter Restaurationswerkstoffe und beauftragten Laboratorien auf die Passgenauigkeit analysiert werden.

Folgende Nullhypothese wurde geprüft:

Hauptzielkriterium: Die Passgenauigkeit der Kronen auf Basis digitaler Abformung und die der auf konventionellen Abformungen basierenden Kronen ist gleich.

Nebenzielkriterien: Restaurationswerkstoffe (A) und Laboratorien (B) haben keinen Einfluss auf die Passgenauigkeit. 


\section{Material und Methode}

\subsection{Urmodell}

Zur Durchführung dieser in vitro Studie wurde zunächst ein Urmodell konstruiert. Als Vorlage für die Anfertigung diente ein KaVo-Übungsmodell (KaVo Dental GmbH, Biberach). Bei dem Urmodell handelte es sich um ein Oberkiefer-Modell, bei dem die Zähne 18-28 aus Edelstahl angefertigt wurden. Der Zahnkranz wurde auf einer Stahlplatte fixiert und anschließend in Gips gefasst. Zahn 16 stellte den Urstumpf dar. Basierend auf der zuvor angefertigten Kronenpräparation am KaVo-Modell durch eine approbierte Zahnärztin (Verfasserin) wurde der Stumpf in einer Präzisionswerkstatt gefräst. Er bestand aus einem Metallpfeiler, der mit einer Hohlkehlpräparationsgrenze versehen war, zirkulär $2 \mathrm{~mm}$ planparallele Flächen aufwies und 2,5mm nach okklusal hin konisch ( $6^{\circ}$ Konuswinkel) zusammenlief (Abb.4).

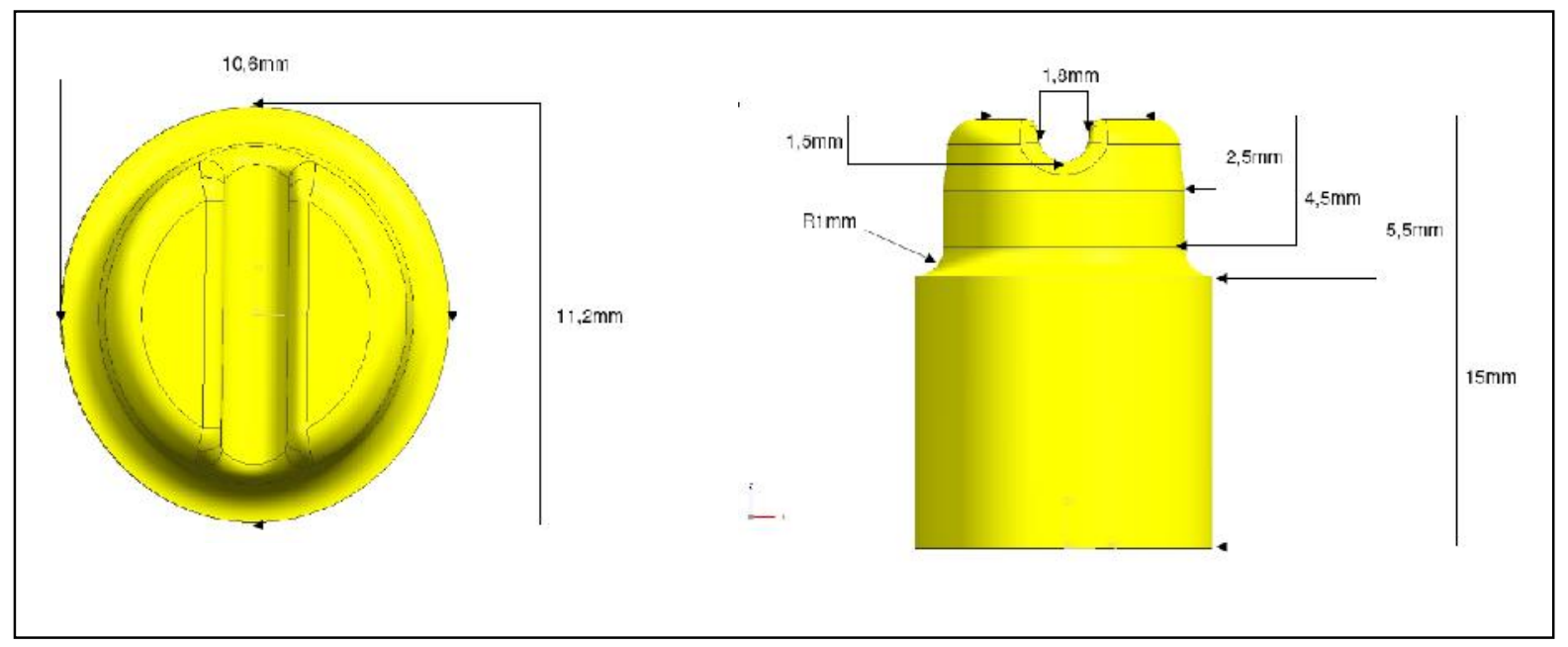

Abb.4: Schematische Darstellung des Urstumpfs

Die planparallelen Flächen dienten zur Ermittlung der Genauigkeit, mit der ein einzelner Stumpf reproduziert wurde. Der Pfeiler wurde okklusal mit einer zentrischen Rille versehen, die zur Rotationssicherung der Kronen auf dem Pfeiler diente. Der Urstumpf 16 war, ebenso 
wie die Nachbarzähne 15 und 17, in einen rechteckigen Kasten gefasst, der für jeden Zahn ein separates Fach aufwies. Der Urstumpf 16 war mit einer Schraube auf der Stahlplatte befestigt und lies sich aus dem „Zahnfach“ herausschrauben (Abb.5 und 6).

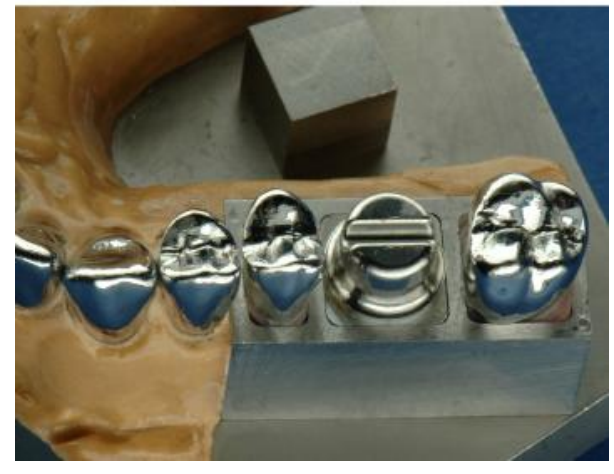

Abb.5: Urstumpf 16

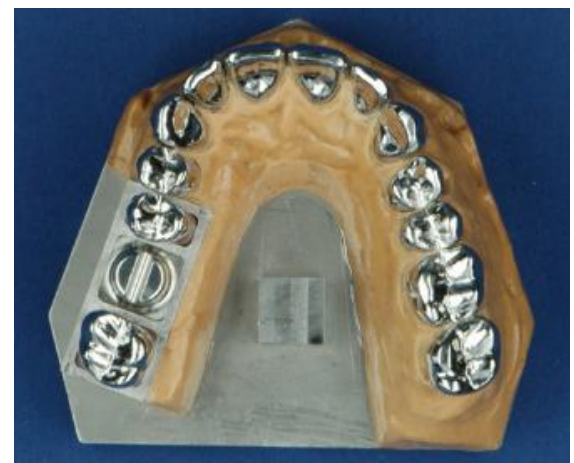

Abb.6: Oberkieferurmodell mit Urstumpf 16

Zur Durchführung der digitalen Abformungen wurde ein zweiter Urstumpf hergestellt, der sich in seiner Ausführung nur durch eine matte Oberfläche vom Urstumpf für die konventionelle Abformung unterschied (Abb.7 und 8).

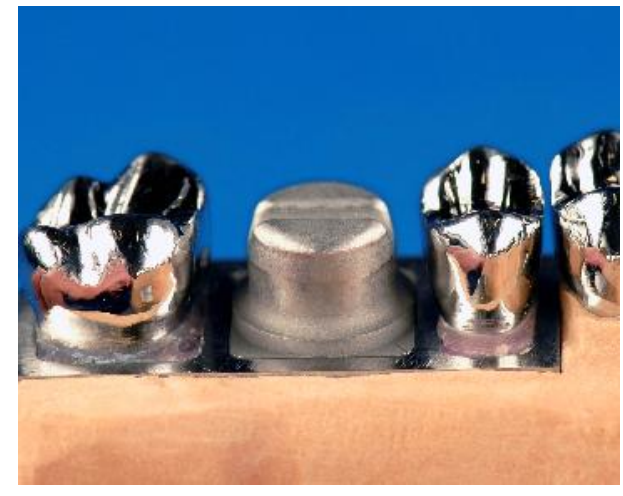

Abb.7: Detailaufnahme des Scan-Urstumpfes

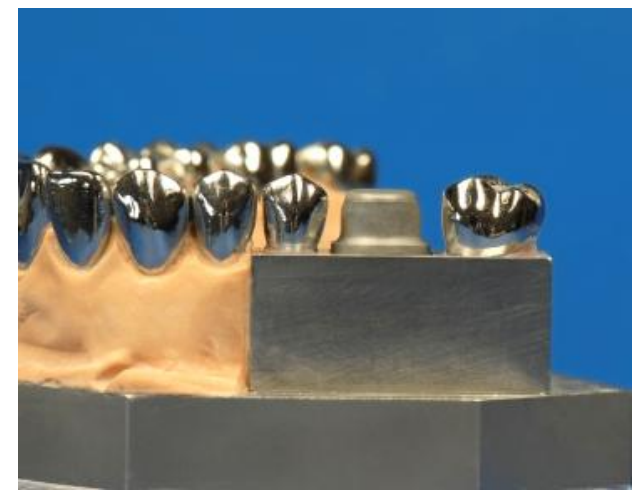

Abb.8: Urstumpf mit matter Oberfläche 
Die matte Stumpfoberfläche diente der Vermeidung von Fehlern durch übermäßige Reflexionen von der Stumpfoberfläche. Zusätzlich wurde vor dem Scanvorgang der Stumpf zur Schaffung einer kontrastreichen Oberfläche mit einem speziellen Scan-Puder bestäubt (Abb.9). Als Puder diente das jeweils vom Hersteller empfolene Kontrastpuder mit dem Hauptbestandteil Titandioxid, dass mit Hilfe einer Sprühpistole gleichmäßig appliziert wurde.

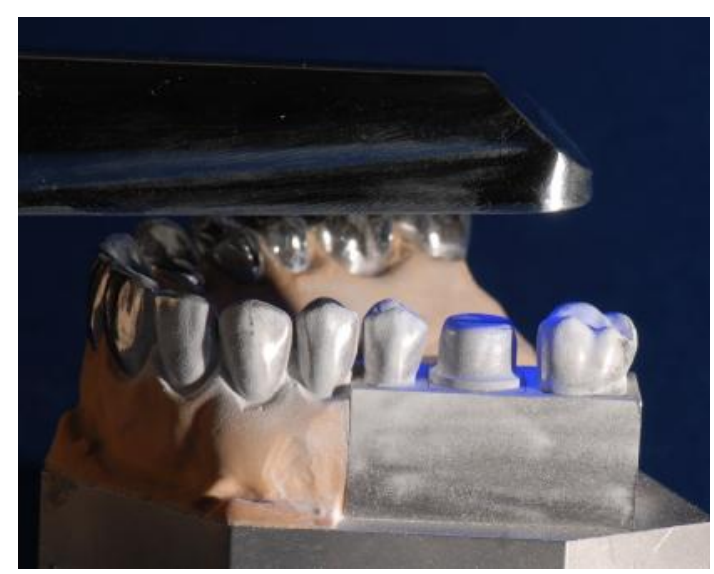

Abb.9: Scanvorgang mit LCOS

Für den Gegenkiefer wurden ebenfalls Zähne aus Edelstahl in Gips gefasst. Das OberkieferUrmodell und das Unterkiefer-Urmodell wurden in zentraler Okklusion einartikuliert (Abb.10).

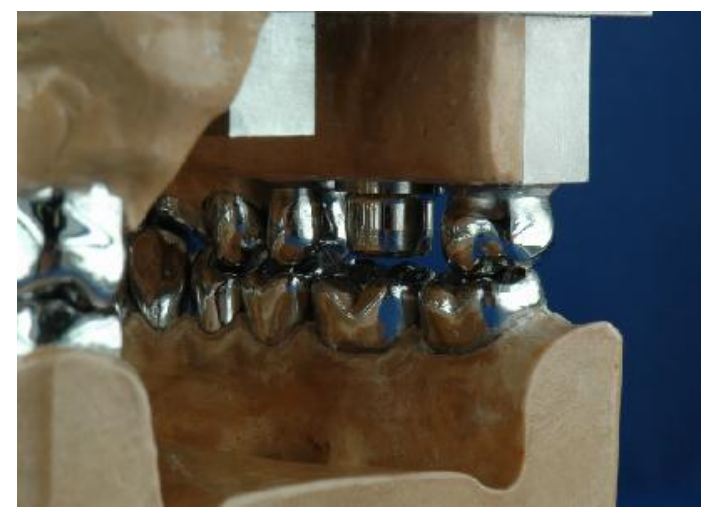

Abb.10: Urmodell in zentraler Okklusion 


\subsection{Durchführung der Abformungen und Herstellung der Prüfkörper}

Zur Herstellung der Prüfkörper (Kronen/Kronengerüste) wurde das Urmodell im ersten Schritt konventionell ( $n=50)$ und im zweiten Schritt optisch abgeformt $(n=30)$. Alle Abformungen sowie alle Scanvorgänge wurden bei $23^{\circ} \mathrm{C} \pm 1^{\circ} \mathrm{C}$ unter standardisierten Bedingungen von approbierten Zahnärzten (Verfasserin und ein weiterer, kalibrierter Zahnarzt) durchgeführt. Alle anschließenden Vermessungen wurden von der Verfasserin dieser Dissertation durchgeführt, um personenspezifische Messfehler zu vermeiden. Alle Materialien wurden nach Herstellerangaben verarbeitet. Zur Durchführung der konventionellen Abformungen wurden ausschließlich A-Silikone verwendet. Als Abformlöffel wurden verwindungssteife, perforierte (Korrekturabformung) sowie glatte (Doppelmischabformung) Metall-Serienlöffel der Größe 2 (Aesculap) verwendet. Das Anmischen des Putty-Materials erfolgte maschinell mit einem Pentamix-II-Anmischgerät (3M Espe, Seefeld). Die Vorabformungen, bei Durchführung der Korrekturtechnik, wurden nach der Löffelentnahme ausgeschnitten.

Bei Durchführung der Korrekturphase wurde der Löffel unmittelbar nach Aufbringen des Korrekturmaterials in Sollposition gebracht, fünf Sekunden angedrückt, im Anschluss härtete das Abformmaterial drucklos aus. Die Applikation der dünnfließenden Phase erfolgte bei der Doppelmischabformung gleichzeitig mit dem Putty-Material jeweils am Urstumpf und auf dem Löffelmaterial (modifizierte Doppelmischabformung). Die Abformlöffel mit den Abformmassen wurden manuell auf dem Urmodell positioniert. Tabelle 1 zeigt eine Übersicht der verwendeten Abformmaterialien.

Tabelle 1: Übersicht über die verwendeten Materialien bei den Silikon-Abformungen

\begin{tabular}{|l|c|c|}
\hline Abformtechnik & Putty Material & dünnfließendes Material \\
\hline Doppelmischtechnik & 3M Espe; Express 2 Penta Putty & 3M Espe; Express 2 Light Body \\
& LOT Nr.: 384177/384971 & Standard \\
& & LOT Nr.: 380640 \\
\hline Korrekturtechnik & 3M Espe; Express 2 Penta Putty & 3M Espe; Express 2 Light Body \\
& LOT Nr.: 384177/384971 & Standard \\
& & LOT Nr. 380640 \\
\hline
\end{tabular}


Im Anschluss wurden die 50 Abformungen an 2 unterschiedlichen Dentallaboratorien versandt und nach einer Lagerungszeit von 24 Stunden jeweils mit einem Typ IV Gips ausgegossen, um reproduzierbare Modelleigenschaften zu gewährleisten.

Das Dentallabor A (Tabelle 2) erhielt 30 Abformungen in Korrekturtechnik aus denen 30 Gipsmodelle angefertigt wurden. Auf diesen wurden 30 Kronen aus drei unterschiedlichen Materialien (10 x Zirkonoxid, 10 x goldhaltige Legierung, 10 x Kobalt-Chrom Legierung; jeweils keramisch vollverblendet) hergestellt.

Labor B (Tabelle 2) erhielt 10 Abformungen in Korrekturtechnik und 10 Abformungen in Doppelmischtechnik aus denen je 10 Gipsmodelle und darauf je 30 Kronengerüste aus drei unterschiedlichen Materialien (20 x LAVA Zirkonoxid, 20 x Nacera Zirkonoxid, 20 x goldhaltige Legierung) angefertigt wurden.

Anschließend wurden 10 Scans mit dem LCOS und 20 Scans mit der CEREC-Bluecam vorgenommen. Nach Überprüfung des Scannergebnisses durch die jeweilige systemeigene Software wurden die erstellten Datensätze von LCOS zur Weiterverarbeitung zuerst an ein 3M Espe-Zentrum in den USA versand. Im Eigenlabor unserer Poliklinik (Labor D) und im zahntechnischen Labor C (Tabelle 2), an das 10 Datensätze von CEREC AC übermittelt wurden, sind die Daten jeweils digital bearbeitet worden (Inlab 3.80, Sirona Dental Systems GmbH, Bensheim) und es wurden je 10 Kronen (Labor C: Empress CAD; Labor D: Vita Mark II) gefräst (CEREC Inlab MCXL/CEREC MCXL, Sirona Dental Systems GmbH, Bensheim). Im 3M Espe-Zentrum erfolgte nach computergestützter Aufarbeitung der Messergebnisse zunächst die Herstellung von Kunststoffmodellen im stereolitographischen Verfahren (SLA). Die gescannten Daten wurden dann ebenfalls an Labor C und zusätzlich an das Labor B versandt und digital nachbearbeitet (Openscan, Open-Cut, Laserdata, Bergheim). Hierauf erfolgten anschließend das Design (Dental-CAM + Dental-CNC, VHF-Camfacture AG, Ammerbuch) und die Herstellung von je 10 Zirkonoxid-Messkäppchen auf einer 3+1-achsigen Fräsmaschine (VHF-Impression, VHF-Camfacture AG, Ammerbuch). In Tabelle 3 wird ein Überblick über alle, in dieser Untersuchung hergestellten, Kronen gegeben.

Tabelle 2: Hersteller der Prüfkörper

\begin{tabular}{|l|l|}
\hline Labor A & Permadental, S’Heerenberg, Niederlande \\
\hline Labor B & Peter Kappert, Essen \\
\hline Labor C & Jörg Pohl, Senden \\
\hline Labor D & Eigenlabor, Zahnärztliche Prothetik, Giessen \\
\hline
\end{tabular}


Tabelle 3: Überblick über die Herstellung der Prüfkörper

\begin{tabular}{|c|c|c|c|c|c|}
\hline Abformtechnik & $\begin{array}{l}\text { Anzahl } \\
\text { Abformun- } \\
\text { gen }\end{array}$ & $\begin{array}{l}\text { Anzahl } \\
\text { Kronen }\end{array}$ & Material der Kronen & Hersteller & Labor \\
\hline \multirow[t]{3}{*}{$\begin{array}{l}\text { Doppelmischab- } \\
\text { formung }\end{array}$} & 10 & 10 & $\begin{array}{l}\text { Cera E (Silber- } \\
\text { Palladium-Legierung) }\end{array}$ & $\begin{array}{l}\text { Elephant Dental } \\
\text { B.V., Niederlande }\end{array}$ & B \\
\hline & & 10 & LAVA Zirkon & 3M Espe, MN, USA & B \\
\hline & & 10 & Nacera Zirkon & $\begin{array}{l}\text { Doceram GmbH, } \\
\text { Deutschland }\end{array}$ & B \\
\hline \multirow[t]{6}{*}{$\begin{array}{l}\text { Korrekturabfor- } \\
\text { mung }\end{array}$} & 40 & 10 & $\begin{array}{l}\text { Cera E (Silber- } \\
\text { Palladium-Legierung) }\end{array}$ & $\begin{array}{l}\text { Elephant Dental } \\
\text { B.V., Niederlande }\end{array}$ & B \\
\hline & & 10 & LAVA Zirkon & 3M Espe, MN, USA & B \\
\hline & & 10 & Nacera Zirkon & $\begin{array}{l}\text { Doceram GmbH, } \\
\text { Deutschland }\end{array}$ & B \\
\hline & & 10 & $\begin{array}{l}\text { D.Sign } 98 \text { (hochgold- } \\
\text { haltige Legierung) } \\
\text { GCInitialMC- } \\
\text { DA2/S58/DA3/S59 }\end{array}$ & $\begin{array}{l}\text { Ivoclar Vivadent AG, } \\
\text { Liechtenstein } \\
\text { Klema GmbH, Öster- } \\
\text { reich }\end{array}$ & A \\
\hline & & 10 & $\begin{array}{l}\text { Wirobond } 280 \text { (Kobalt- } \\
\text { Chrom Legierung) } \\
\text { GCInitial- } \\
\text { MCDA2/S58/DA3/S59 }\end{array}$ & $\begin{array}{l}\text { Bego GmbH\& Co. } \\
\text { KG, Deutschland } \\
\text { Klema GmbH, Ös- } \\
\text { terreich }\end{array}$ & A \\
\hline & & 10 & $\begin{array}{l}\text { Nacera Zirkon } \\
\text { Vintage- } \\
\text { DA2/S58/DA3/S59 }\end{array}$ & $\begin{array}{l}\text { Doceram GmbH, } \\
\text { Deutschland } \\
\text { Shofu GmbH, Japan }\end{array}$ & A \\
\hline \multirow[t]{2}{*}{$\begin{array}{l}\text { Intraoraler Scan } \\
\text { LAVA C.O.S. }\end{array}$} & 10 & 10 & LAVA Zirkon & 3M Espe, MN, USA & B \\
\hline & & 10 & LAVA Zirkon & 3M Espe, MN, USA & $\mathrm{C}$ \\
\hline \multirow[t]{2}{*}{$\begin{array}{l}\text { Intraoraler Scan } \\
\text { CEREC 3D }\end{array}$} & 20 & 10 & Empress CAD & $\begin{array}{l}\text { Ivoclar Vivadent } \\
\text { AG, Liechtenstein }\end{array}$ & $\mathrm{C}$ \\
\hline & & 10 & Vita Mark II & $\begin{array}{l}\text { Vita Zahnfabrik, } \\
\text { Deutschland }\end{array}$ & $\mathrm{D}$ \\
\hline
\end{tabular}


In den folgenden Tabellen (Tabelle 4-7) sind die Herstellungsparameter aller gefrästen Keramikkronen, unterschieden nach den verschiedenen Werkstoffen, zusammengefasst.

Tabelle 4: Scan/ Herstellungsparameter Nacera- Zirkon Kronen

\begin{tabular}{|c|c|c|}
\hline \multicolumn{3}{|l|}{ Nacera Zirkon } \\
\hline Materialien/Hardware/Software & Produkt & Hersteller \\
\hline Dentallabor-Scanner & $\begin{array}{l}\text { Openscan 100, 5-axige Ma- } \\
\text { schine }\end{array}$ & $\begin{array}{l}\text { Laserdenta, Bergheim, Deutsch- } \\
\text { land }\end{array}$ \\
\hline Scan software & Openscan 3.0, & $\begin{array}{l}\text { Laserdenta, Bergheim, Deutsch- } \\
\text { land }\end{array}$ \\
\hline CAD-Software & $\begin{array}{l}\text { Open-CAD } 2.0 \\
(1 . \text { Serie/2.Serie }->3.0)\end{array}$ & $\begin{array}{l}\text { Laserdenta, Bergheim, Deutsch- } \\
\text { land }\end{array}$ \\
\hline CAM-Software & Dental-CAM 2.0 & $\begin{array}{l}\text { VHF Camfacture AG, Ammer- } \\
\text { buch }\end{array}$ \\
\hline CNC-Software & Dental-CNC 2.0 & $\begin{array}{l}\text { VHF Camfacture AG, Ammer- } \\
\text { buch }\end{array}$ \\
\hline \multicolumn{2}{|l|}{ Herstellungsparameter: } & \\
\hline \multicolumn{2}{|c|}{$=0,08 \mathrm{~mm}$} & \\
\hline CNC-Fräsmaschine & $\begin{array}{l}\text { VHF impression model } 4 / 02 \\
(3+1 \text {-axis machine }),\end{array}$ & $\begin{array}{l}\text { VHF Camfacture AG, Ammer- } \\
\text { buch }\end{array}$ \\
\hline Zirkonrohling & Nacera Z & Doceram GmbH, Dortmund, \\
\hline Sinterofen & LAVA Furnace 200 & 3M Espe, St Paul, MN, USA \\
\hline
\end{tabular}


Tabelle 5: Scan/ Herstellungsparameter für LAVA Zirkon

\begin{tabular}{|c|c|c|}
\hline \multicolumn{3}{|l|}{ LAVA Zirkon } \\
\hline Materials/Hardware/Software & Produkt & Hersteller \\
\hline Dentallabor-Scanner & LAVA Scan ST & 3M Espe, St Paul, MN, USA \\
\hline \multicolumn{3}{|l|}{ Scan Software } \\
\hline CAD-Software & LAVA Design Software & 3M Espe, St Paul, MN, USA \\
\hline \multicolumn{3}{|l|}{ CAM-Software } \\
\hline \multicolumn{3}{|l|}{ CNC-Software } \\
\hline \multicolumn{2}{|l|}{ Herstellungsparameter: } & \\
\hline Zementspalt & $=0,02 \mathrm{~mm}$ & \\
\hline CNC-Fräsmaschine & $\begin{array}{l}\text { LAVA Form M347 } \\
\text { (3+1-axige Maschine) }\end{array}$ & 3M Espe, St Paul, MN, USA \\
\hline Zirkonrohling & LAVA Zirkon & 3M Espe, St Paul, MN, USA \\
\hline Sinterofen & LAVA Furnace 200 & 3M Espe, St Paul, MN, USA \\
\hline
\end{tabular}

Tabelle 6: Scan/ Herstellungsparameter für IPS Empress CAD

\begin{tabular}{|c|c|c|}
\hline \multicolumn{3}{|l|}{ Empress CAD } \\
\hline Materialien/Hardware/Software & Produkt & Hersteller \\
\hline CAD/CAM-Software & Inlab 3.80 & $\begin{array}{l}\text { Sirona Dental Systems GmbH, } \\
\text { Bensheim, Deutschland }\end{array}$ \\
\hline \multicolumn{3}{|l|}{ Herstellungsparameter: } \\
\hline Zementspalt & $=0,02 \mathrm{~mm}$ & \\
\hline \multirow[t]{2}{*}{ CNC-Fräsmaschine } & CEREC Inlab & $\begin{array}{l}\text { Sirona Dental Systems GmbH, } \\
\text { Bensheim, Deutschland }\end{array}$ \\
\hline & CEREC MCXL & $\begin{array}{l}\text { Sirona Dental Systems GmbH, } \\
\text { Bensheim, Deutschland }\end{array}$ \\
\hline
\end{tabular}


Tabelle 7: Scan/ Herstellungsparameter für Vita MarkII

\begin{tabular}{|c|c|c|}
\hline \multicolumn{3}{|l|}{ Vita MarkII } \\
\hline Materialien/Hardware/Software & Produkt & Hersteller \\
\hline CAD/CAM-Software & Inlab 3.80 & $\begin{array}{l}\text { Sirona Dental Systems GmbH, } \\
\text { Bensheim, Deutschland }\end{array}$ \\
\hline \multicolumn{3}{|l|}{ Herstellungsparameter: } \\
\hline Zementspalt & $=0,04 \mathrm{~mm}$ & \\
\hline CNC-Fräsmaschine & CEREC MCXL & $\begin{array}{l}\text { Sirona Dental Systems GmbH, } \\
\text { Bensheim, Deutschland }\end{array}$ \\
\hline
\end{tabular}




\subsection{Untersuchungsparameter}

Die Prüfkörper wurden nun im Bezug auf ihre Passgenauigkeit auf dem Urmodell in zwei unterschiedlichen Verfahren geprüft.

\subsubsection{Rundheit}

Als erstes wurde die innere Rundheit aller Kronen bestimmt. Unter dem Begriff der Rundheit (Rundheitstoleranz) versteht man eine Formtoleranz die sich auf Kreise und Kreisquerschnitte bezieht. Die Rundheitstoleranz legt fest, dass in jedem senkrechten Schnitt zur Achse des jeweiligen tolerierten, kegelförmigen Elementes die Umfangslinie zwischen zwei in derselben Ebene liegenden konzentrischen Kreisen mit dem radialen Abstand t (Abb.11) liegen muss ${ }^{34}$, 42,60 .

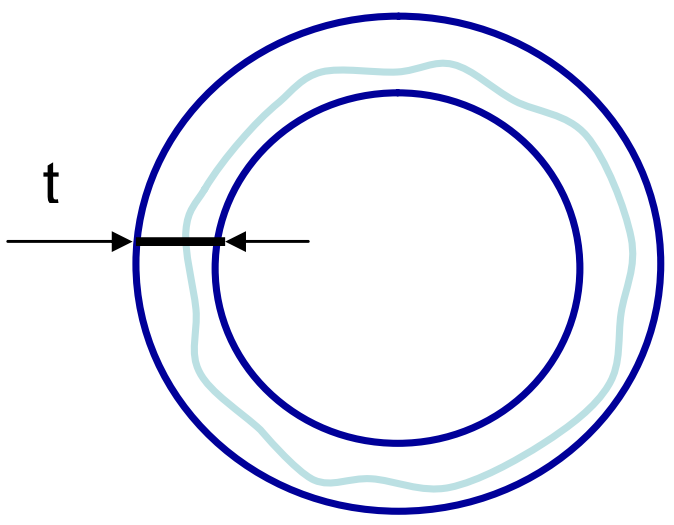

Abb.11: Rundheit; B. Klein 2005

Alle Kronen wurden dazu mit dem Lumen nach oben in Knetmasse auf dem Messtisch der Messmaschine fixiert. Mit Hilfe einer Wasserwaage wurden die Kronen parallel zum Messtisch ausgerichtet, um Messungenauigkeiten auszuschalten. Die Kronen wurden mit einer 3DKoordinaten-Messmaschine (CNC Rapid, THOME GmbH, Messel, Messmaschinennummer: P 654203, Baujahr:04/ 2007) vermessen (Abb.13). Die Messgenauigkeit der Maschine wird laut Hersteller mit $\pm 2,5 \mu \mathrm{m}$ angegeben. Mit einem Rubintaster von 1,5mm Durchmesser wurden die Lumen der Kronen angetastet (Abb.12) und die dabei ermittelten X-, Y- und Z- 
Koordinaten jedes einzelnen Punktes von der Software (Metrolog XG, Version 12; Metrologic Group S.A., Frankreich) zu einem geometrischen Element verarbeitet. Es wurden die Hauptscheitelachse (ADrm), Nebenscheitelachse (BDrm) und der Formfehler (der weiteste Punkt der von der idealen Ellipse abweicht in Millimetern) der Ellipse im Kroneninneren bestimmt. Zuerst wurden die Kronen an fünf manuellen und danach automatisch an je 50 Stellen durch die Maschine angetastet. Der Messvorgang wurde pro Einzelkrone zehnmal wiederholt. Im Anschluss wurde der Stumpf 16 des Urmodells in gleicher Verfahrenstechnik zirkulär angetastet und vermessen. Die Durchmesser $($ ADrm $=$ Hauptachse, BDrm $=$ Nebenachse $)$ und die jeweiligen Formfehler (F.F.) der Kronen wurden mit denen des Stumpf-Urmodells verglichen. Für alle späteren Auswertungen wurde der Wert des Formfehlers herangezogen, da er als Maß für die Abweichung der Rundheit (=t in Abb.11) angesehen werden kann.

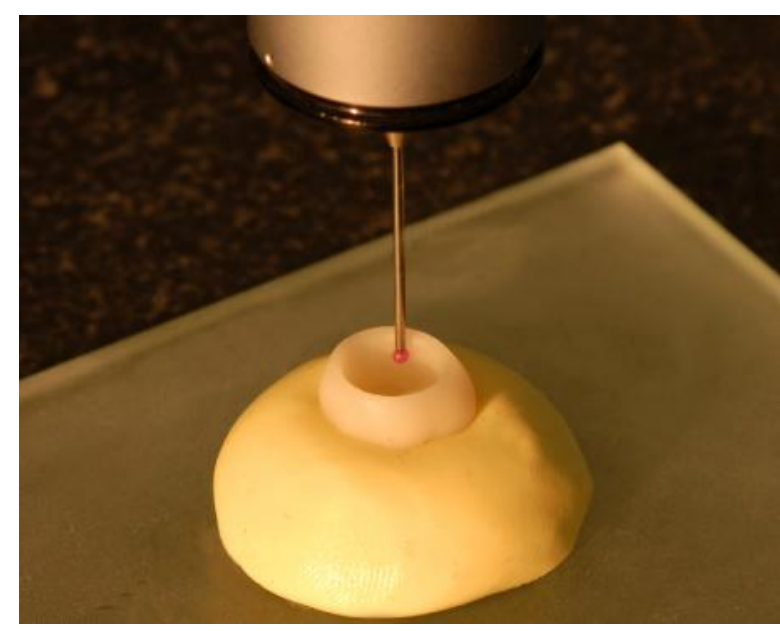

Abb.12: Krone zur Vermessung platziert; Taster im Lumen

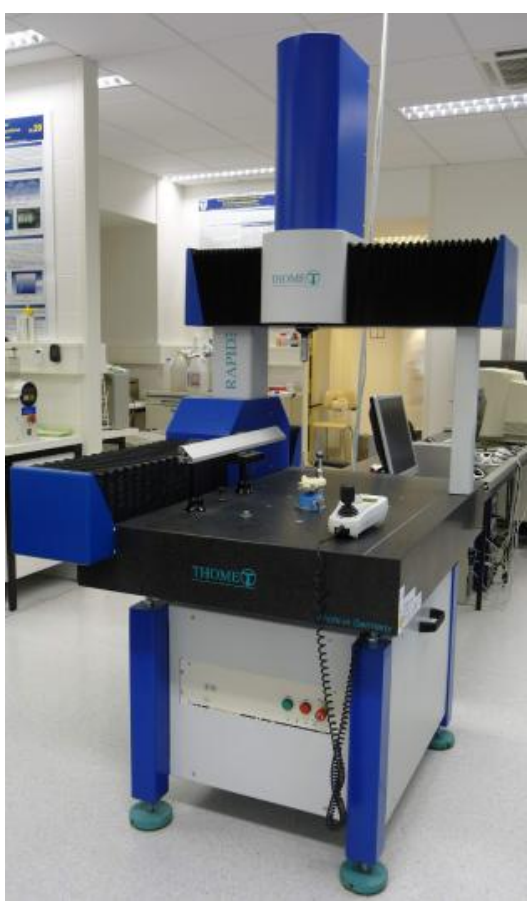

Abb.13: 3D-Koordinaten-Messmaschine 


\subsubsection{Absolute marginale Diskrepanz}

Als zweiter Untersuchungsparameter wurde mit einem Messmakroskop M420 (Leica, Bensheim, 20 bis 80fache Vergrößerung; Reproduzierbarkeit der Einzelmessung $\pm 10 \mu \mathrm{m})$ die absolute marginale Diskrepanz ${ }^{32}$ aller Kronen auf dem jeweiligen Urstumpf nach der 1989 von Holmes publizierten Methode bestimmt (Abb.14). Dabei wurde die in Abb.2 mit ,g“ gekennzeichnete Strecke, die absolute marginale Diskrepanz, als Messstrecke verwendet. Bevor diese Messungen durchgeführt werden konnten, mussten die Kronen jeweils einzeln auf den Urstumpf aufgepasst werden. Dies erfolgte mittels eines feinkörnigen Diamanten (379 EF. 314. 018 Gebr. Brasseler) unter Wasserkühlung durch die Verfasserin. Das optische Messgerät verfügt über ein Makrozoom (6,3-32fach) sowie ein Binokkular 21B (10fach) und ermöglicht somit eine 40fache Vergrößerung. Zur Aufnahme der gemessenen Streckenabschnitte in Xund Y-Richtung sind dem Mikroskop zwei Mikrometer-Messuhren als Wegaufnehmer angeschlossen (Mitutoyo Digimatic, Mitutoyo Corp. Japan) (Abb.15). Der Urstumpf konnte auf einer eigens für diese Messungen angefertigten Halterung in einem Winkel von $35^{\circ}$ fixiert werden (Abb.16). Dieser Winkel erlaubte eine optimale Aufsicht auf den Kronenrand. Es wurde pro Krone jeweils an vier definierten Stellen gemessen: mesial, bukkal, distal und palatinal.

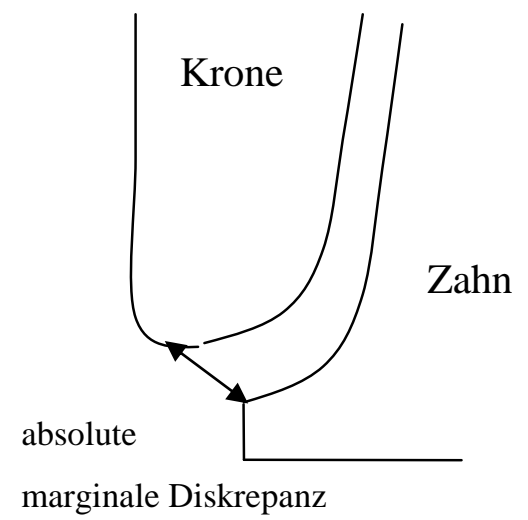

Abb.14: Absolute marginale Diskrepanz nach Holmes et al. ${ }^{32}$ 


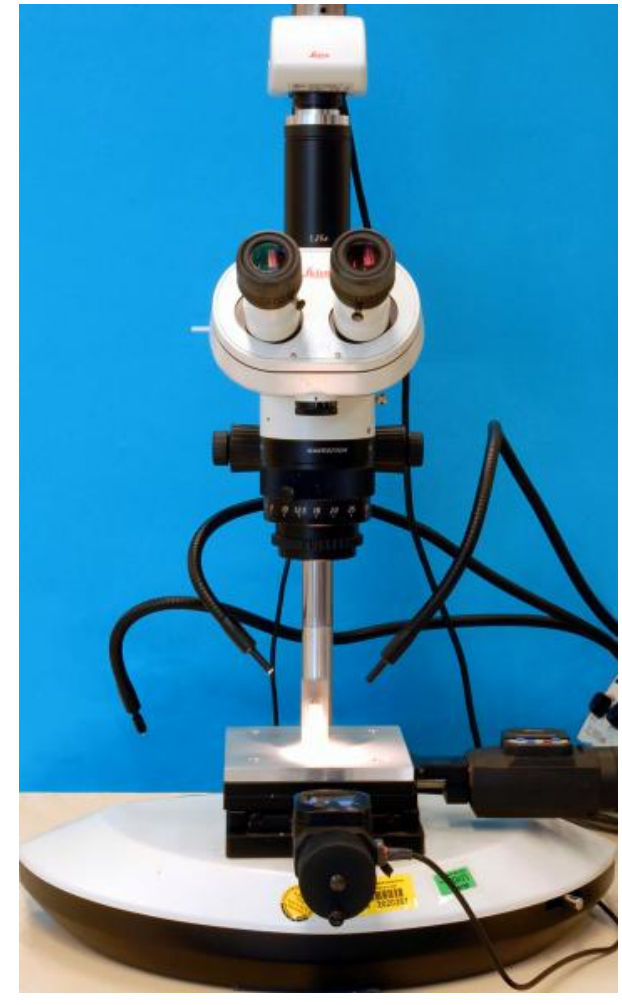

Abb.15: Messmakroskop M420

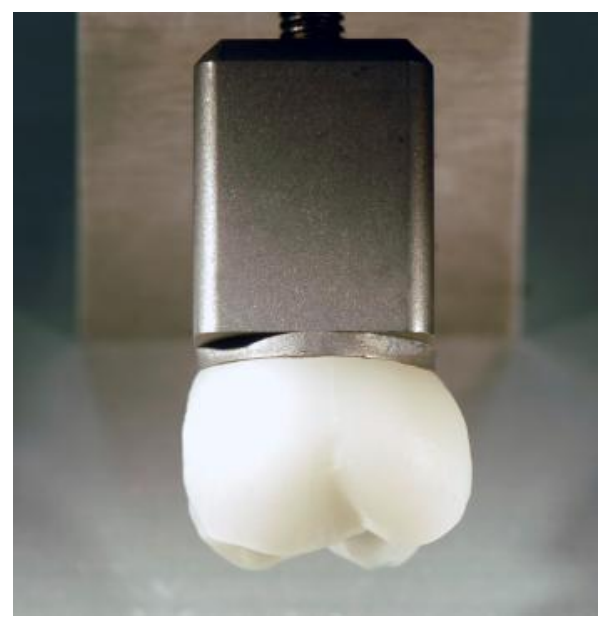

Abb.16: Krone auf Urstumpf zur Vermessung

\subsection{Statistische Analyse}

Alle erhobenen Messwerte wurden zunächst in Excel bearbeitet und zusammengefasst. Zur Durchführung der statistischen Auswertung wurde die SPSS Software, Version 15.0 (SPSS GmbH Software, München, Deutschland) verwendet. Die Auswertung beruhte auf korrelativen und varianzanalytischen, teststarken Verfahren. Das Hauptaugenmerk lag auf der Ermittlung der jeweiligen Mittelwerte. Als erstes wurde eine deskriptive Auswertung der Messwerte in Form eines Gruppenvergleiches vorgenommen. Im Anschluss wurde eine Varianzanalyse in Form eines Levene-Tests $(\mathrm{p}=0,05)$ durchgeführt. Da nicht für alle Gruppen Varianzhomogenität gegeben war, wurde im Anschluss als Post-Hoc-Test der Games-Howell Test (p=0,05) angewendet. Zur besseren Visualisierung wurden im Ergebnisteil ergänzend Boxplotdiagramme gewählt.

In der folgenden Tabelle 8 wird eine Übersicht der vorhandenen Daten gegeben. 
Tabelle 8: Überblick über die vorhandenen Daten

\section{Verteilung der Prïfkörper ( Fallzahlen)}

\begin{tabular}{|c|c|c|c|c|c|c|}
\hline \multicolumn{7}{|c|}{ Abformverfahren } \\
\hline Labor & Material & $\begin{array}{l}\text { LCOS- } \\
\text { Scan }\end{array}$ & $\begin{array}{l}\text { CEREC- } \\
\text { Scan }\end{array}$ & $\begin{array}{l}\text { Doppelmisch- } \\
\text { abformung }\end{array}$ & $\begin{array}{l}\text { Korrektur- } \\
\text { abformung }\end{array}$ & Gesamt \\
\hline $\mathbf{D}$ & Vita Mark II & & 10 & & & 10 \\
\hline \multirow[t]{2}{*}{ C } & Lava & 10 & & & & 10 \\
\hline & $\begin{array}{l}\text { Empress } \\
\text { CAD }\end{array}$ & & 10 & & & 10 \\
\hline \multirow[t]{3}{*}{ A } & EMF & & & & 10 & 10 \\
\hline & Nacera & & & & 10 & 10 \\
\hline & Gold & & & & 10 & 10 \\
\hline \multirow[t]{3}{*}{ B } & Lava & 10 & & 10 & 10 & 30 \\
\hline & Nacera & & & 10 & 10 & 20 \\
\hline & Gold & & & 10 & 10 & 20 \\
\hline Gesamt & & 20 & 20 & 30 & 60 & 130 \\
\hline
\end{tabular}




\section{Ergebnisse}

\subsection{Gesamtübersicht der Ergebnisse}

\subsubsection{Rundheit: Bestimmung des Formfehlers}

Die Ermittlung des Formfehlers ergab die geringsten Werte für die LAVA Zirkon Kronen auf

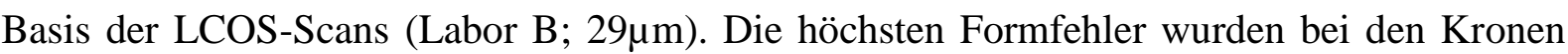
aus dem Werkstoff Vita Mark II, basierend auf CEREC-Scans gemessen (Labor D; 130 $\mu \mathrm{m}$ ). Alle Kronen die auf der Basis konventioneller Abformungen hergestellt wurden, wiesen insgesamt homogene Werte auf. Im Mehrfachvergleich der Messdaten ergaben sich für Kronen, gefertigt auf Basis der CEREC-Scans die signifikant größten Werte für den Formfehler im Vergleich zu allen anderen Kronen. Die Verteilung der Messergebnisse wurde zur Verdeutlichung in dem folgenden Diagramm (Abb.17) dargestellt.

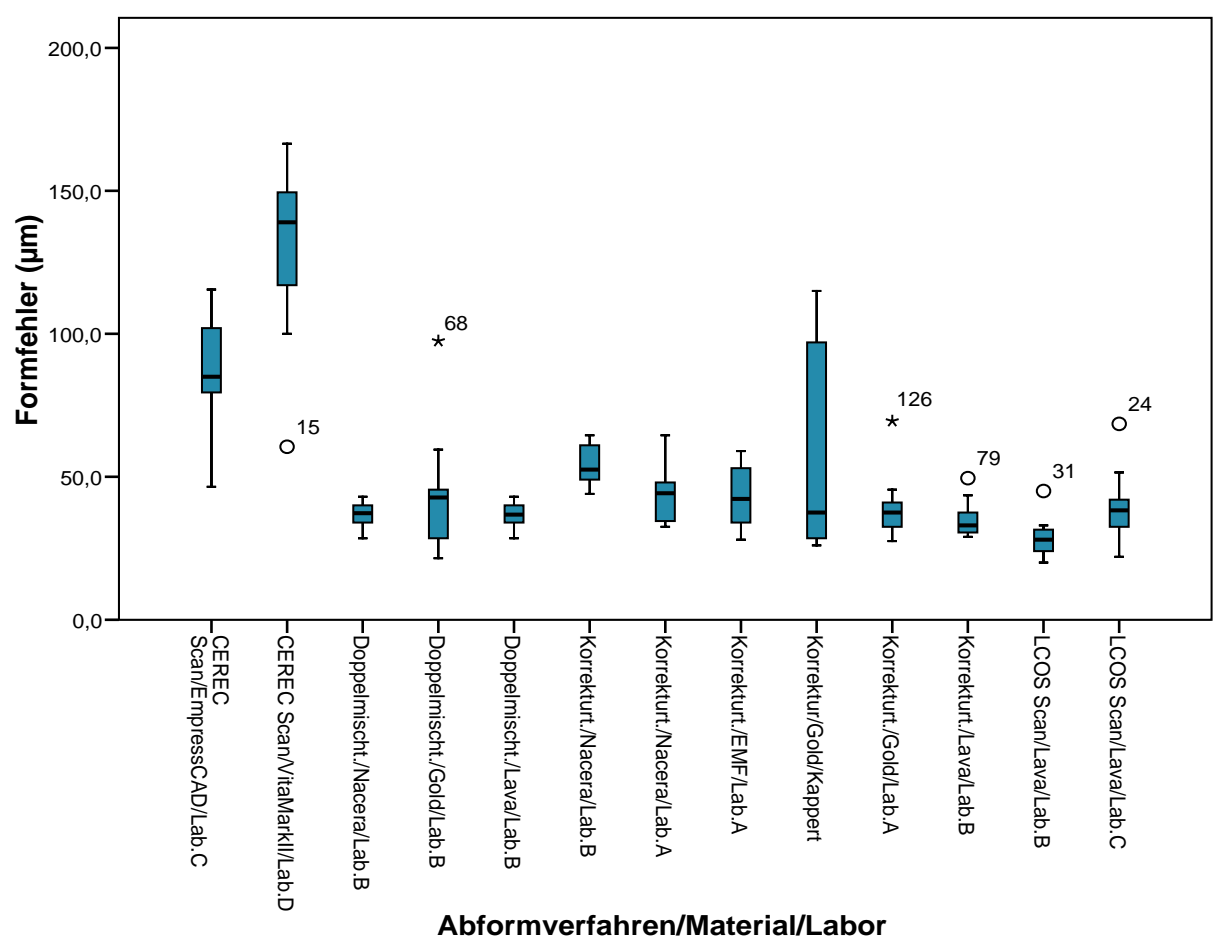

Abb.17: Boxplot Diagramm der Formfehler aller Kronen 
Die Ergebnisse können im Einzelnen der folgenden Tabelle 9 entnommen werden. Die niedrigsten Werte sind in grün, die höchsten in rot hinterlegt.

Tabelle 9: Formfehler in den Vergleichsgruppen

\begin{tabular}{|c|c|c|c|c|c|c|c|c|}
\hline \multicolumn{9}{|c|}{ Formfehler } \\
\hline Labor/Material/Verfahren & $\mathbf{N}$ & $\begin{array}{l}\text { Mittel- } \\
\text { wert } \\
(\mu \mathrm{m})\end{array}$ & $\begin{array}{l}\text { Standard- } \\
\text { abweichung } \\
(\mu \mathrm{m})\end{array}$ & $\begin{array}{l}\text { Standard- } \\
\text { fehler } \\
(\mu \mathrm{m})\end{array}$ & $\begin{array}{l}95 \%- \\
\text { Konfidenzin- } \\
\text { terwall der } \\
\text { Mittelwerte, } \\
\text { Untergrenze } \\
(\mu \mathrm{m})\end{array}$ & $\begin{array}{l}95 \%- \\
\text { Konfidenzin- } \\
\text { terwall der } \\
\text { Mittelwerte, } \\
\text { Obergrenze } \\
(\mu \mathrm{m})\end{array}$ & $\begin{array}{l}\text { Minimum } \\
(\mu \mathrm{m})\end{array}$ & $\begin{array}{l}\text { Maximum } \\
(\mu \mathrm{m})\end{array}$ \\
\hline $\begin{array}{lrl}\text { CEREC } \quad \mathrm{Scan} / & \mathrm{Em}- \\
\text { pressCAD/Labor } \mathrm{C} & \end{array}$ & 10 & 88 & 20 & 6 & 74 & 102 & 47 & 116 \\
\hline $\begin{array}{l}\text { CEREC Scan/ Vita MarkII/ } \\
\text { Labor D }\end{array}$ & 10 & 130 & 31 & 10 & 108 & 152 & 61 & 167 \\
\hline $\begin{array}{l}\text { Doppelmischabformung/ } \\
\text { Nacera/ Labor B }\end{array}$ & 10 & 36 & 5 & 2 & 33 & 40 & 29 & 43 \\
\hline $\begin{array}{l}\text { Doppelmischabformung/ } \\
\text { Gold/ Labor B }\end{array}$ & 10 & 44 & 22 & 7 & 28 & 60 & 22 & 98 \\
\hline $\begin{array}{l}\text { Doppelmischabformung/ } \\
\text { Lava/ Labor B }\end{array}$ & 10 & 36 & 5 & 2 & 33 & 40 & 29 & 43 \\
\hline $\begin{array}{l}\text { Korrekturabformung/ } \\
\text { Nacera/ Labor B }\end{array}$ & 10 & 54 & 7 & 2 & 49 & 59 & 44 & 65 \\
\hline $\begin{array}{l}\text { Korrekturabformung/ } \\
\text { Nacera/ Labor A }\end{array}$ & 10 & 44 & 10 & 3 & 37 & 51 & 33 & 65 \\
\hline $\begin{array}{l}\text { Korrekturabformung/ EMF/ } \\
\text { Labor A }\end{array}$ & 10 & 43 & 11 & 4 & 35 & 51 & 28 & 59 \\
\hline $\begin{array}{l}\text { Korrekturabformung / Gold/ } \\
\text { Labor B }\end{array}$ & 10 & 56 & 36 & 11 & 30 & 82 & 26 & 115 \\
\hline $\begin{array}{l}\text { Korrekturabformung/ Gold/ } \\
\text { Labor A }\end{array}$ & 10 & 40 & 12 & 4 & 31 & 8 & 28 & 70 \\
\hline $\begin{array}{l}\text { Korrekturabformung/ Lava/ } \\
\text { Labor B }\end{array}$ & 10 & 35 & 7 & 2 & 30 & 40 & 29 & 50 \\
\hline LCOS Scan/ Lava/ Labor B & 10 & 29 & 7 & 2 & 23 & 34 & 20 & 45 \\
\hline LCOS Scan/ Lava/ Labor C & 10 & 40 & 13 & 4 & 31 & 49 & 22 & 69 \\
\hline
\end{tabular}




\subsubsection{Absolute marginale Diskrepanz}

Bei der Messung der absoluten marginalen Diskrepanz wurden die geringsten Werte bei den Kronen aus Doppelmischabformungen in Verbindung mit dem Restaurationswerkstoff Nacera Zirkon (Labor B) gemessen $(21 \mu \mathrm{m})$, ebenso wie bei den Kronen aus CEREC-Scan Vita

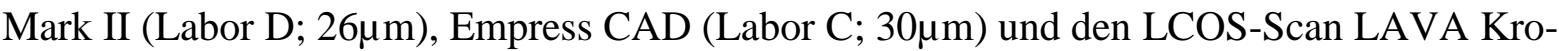
nen (Labor C; $33 \mu \mathrm{m}$ und Labor B; 48 $\mu \mathrm{m}$ ). Die größte marginale Diskrepanz wiesen die Prüfkörper auf, die im Korrekturverfahren abgeformt und aus einer goldhaltigen Legierung hergestellt wurden (Labor B; 68 $\mu \mathrm{m}$ ). Nach Auswertung der Messdaten im Mehrfachvergleich (Games-Howell-Test) ließen sich signifikante Unterschiede, in insgesamt sechs Fällen bei den Kronen aus dem Material Nacera Zirkon (gefertigt auf der Basis von Doppelmischabformungen; Labor B) im Vergleich zu den im Korrekturverfahren abgeformten Kronen feststellen. Die erstgenannten Kronen wiesen hierbei die niedrigeren marginalen Diskrepanzen auf. In dem folgenden Boxplot Diagramm (Abb.18) und der Tabelle 10 werden die Ergebnisse nochmals dargestellt. Die geringste marginale Diskrepanz ist zur Verdeutlichung in der Tabelle in grün hinterlegt, die größte Diskrepanz in rot.

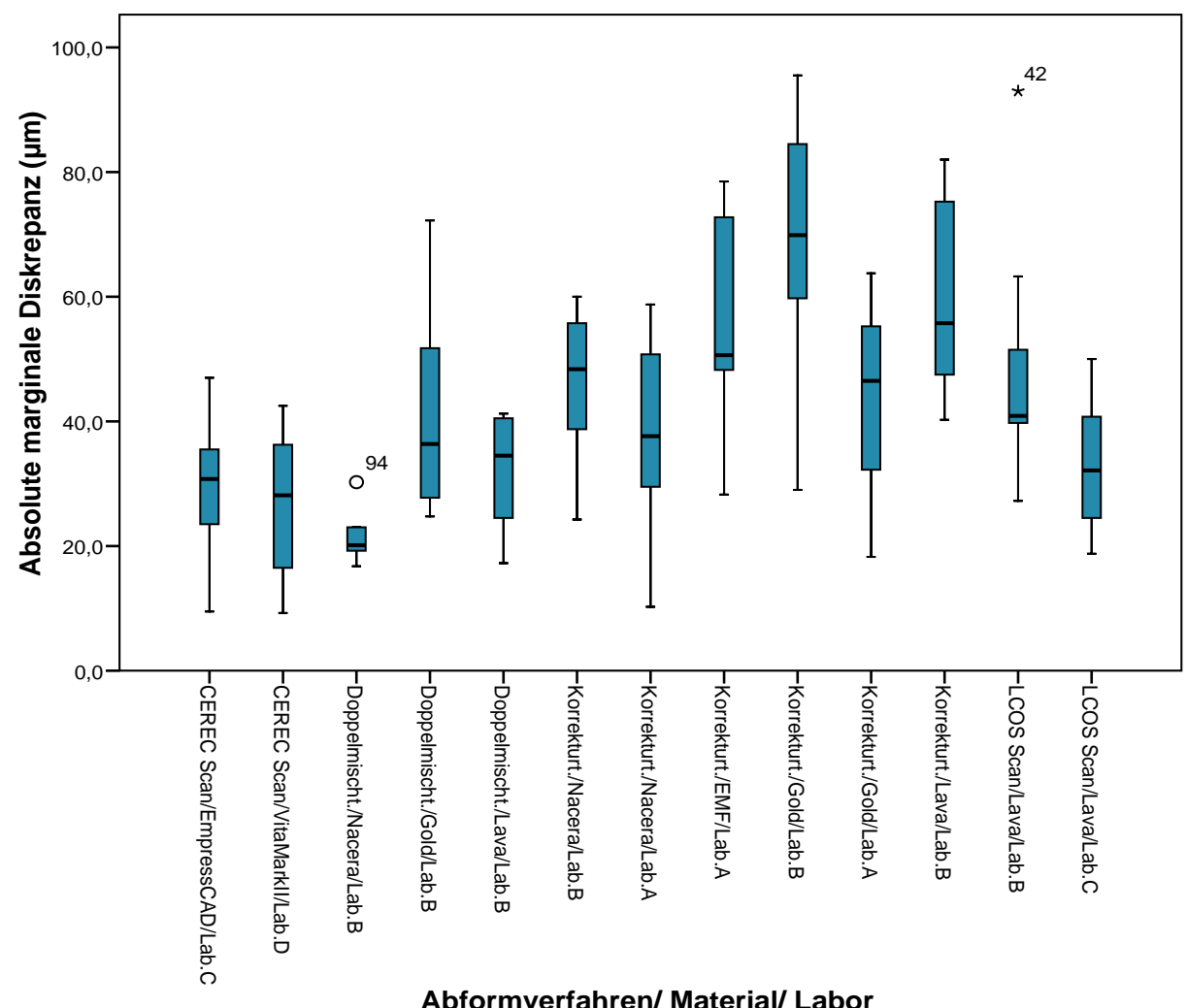

Abb.18: Boxplot- Diagramm der absoluten marginalen Diskrepanz aller Kronen. 
Tabelle 10: Absolute marginale Diskrepanz in den Vergleichsgruppen

\begin{tabular}{|c|c|c|c|c|c|c|c|c|}
\hline \multicolumn{9}{|c|}{ Absolute marginale Diskrepanz } \\
\hline Labor/Material/Verfahren & $\mathbf{N}$ & $\begin{array}{l}\text { Mittel- } \\
\text { wert } \\
(\mu \mathrm{m})\end{array}$ & $\begin{array}{l}\text { Standard- } \\
\text { abweichung } \\
(\mu \mathrm{m})\end{array}$ & $\begin{array}{l}\text { Standard- } \\
\text { fehler } \\
(\mu \mathrm{m})\end{array}$ & $\begin{array}{l}95 \% \text { - } \\
\text { Konfidenzin- } \\
\text { terwall der } \\
\text { Mittelwerte, } \\
\text { Untergrenze } \\
(\mu \mathrm{m})\end{array}$ & $\begin{array}{l}95 \% \text { - } \\
\text { Konfidenzin- } \\
\text { terwall der } \\
\text { Mittelwerte, } \\
\text { Obergrenze } \\
(\mu \mathrm{m})\end{array}$ & $\begin{array}{l}\text { Minimum } \\
(\mu \mathrm{m})\end{array}$ & $\begin{array}{l}\text { Maximum } \\
(\mu \mathrm{m})\end{array}$ \\
\hline $\begin{array}{l}\text { CEREC Scan/ Em- } \\
\text { pressCAD/ Labor C }\end{array}$ & 10 & 30 & 10 & 3 & 22 & 37 & 10 & 47 \\
\hline $\begin{array}{l}\text { CEREC Scan/Vita MarkII/ } \\
\text { Labor D }\end{array}$ & 10 & 26 & 12 & 4 & 18 & 35 & 9 & 43 \\
\hline $\begin{array}{l}\text { Doppelmischabformung/ } \\
\text { Nacera/ Labor B }\end{array}$ & 10 & 21 & 4 & 1 & 19 & 24 & 17 & 30 \\
\hline $\begin{array}{l}\text { Doppelmischabformung/ } \\
\text { Gold/ Labor B }\end{array}$ & 10 & 43 & 17 & 6 & 30 & 55 & 25 & 72 \\
\hline $\begin{array}{l}\text { Doppelmischabformung/ } \\
\text { Lava/ Labor B }\end{array}$ & 10 & 33 & 8 & 3 & 27 & 39 & 17 & 41 \\
\hline $\begin{array}{l}\text { Korrekturabformung/ } \\
\text { Nacera/ Labor B }\end{array}$ & 10 & 46 & 12 & 4 & 37 & 54 & 24 & 60 \\
\hline $\begin{array}{l}\text { Korrekturabformung/ } \\
\text { Nacera/ Labor A }\end{array}$ & 10 & 38 & 15 & 5 & 27 & 49 & 10 & 59 \\
\hline $\begin{array}{l}\text { Korrekturabformung/ EMF/ } \\
\text { Labor A }\end{array}$ & 10 & 55 & 16 & 5 & 43 & 66 & 28 & 79 \\
\hline $\begin{array}{l}\text { Korrekturabformung/ Gold/ } \\
\text { Labor B }\end{array}$ & 10 & 68 & 21 & 7 & 53 & 83 & 29 & 96 \\
\hline $\begin{array}{l}\text { Korrekturabformung/ Gold/ } \\
\text { Labor A }\end{array}$ & 10 & 44 & 16 & 5 & 32 & 55 & 18 & 64 \\
\hline $\begin{array}{l}\text { Korrekturabformung/ Lava/ } \\
\text { Labor B }\end{array}$ & 10 & 60 & 15 & 5 & 49 & 71 & 40 & 82 \\
\hline LCOS Scan/ Lava/ Labor B & 10 & 48 & 18 & 6 & 35 & 61 & 27 & 93 \\
\hline LCOS Scan/ Lava/ Labor C & 10 & 33 & 10 & 3 & 26 & 40 & 19 & 50 \\
\hline
\end{tabular}




\subsection{Ergebnisse des Vergleiches der angewendeten Abformverfahren}

\subsubsection{Formfehler}

Bei der Ermittlung des Formfehlers wiesen die Kronen aus CEREC-Scan / Vita MarkII / Labor D durchweg höhere Werte gegenüber allen anderen Kronen auf. Signifikante Unterschiede bestanden, außer zu CEREC-Scan / Empress CAD / Labor C, in allen Vergleichsgruppen. Auch die Kronen aus CEREC-Scan / Empress CAD / Labor C unterschieden sich durch signifikant hohe Werte von den Kronen aus Doppelmischtechnik, LCOS-Scan und Korrekturtechnik bis auf Korrekturtechnik / Gold / Labor B.

Die Kronen aus LCOS-Scan / LAVA / Labor B zeigten signifikant geringere Formfehler als die Kronen aus Korrekturtechnik / Nacera / Labor B und Labor A.

Zwischen den beiden LCOS-Scan Gruppen bestand kein signifikanter Unterschied.

Beide LCOS-Scan Gruppen wiesen jedoch signifikant geringere Werte auf als alle Kronen aus den CEREC-Scans.

Der Vergleich zwischen den beiden konventionellen Abformtechniken ergab signifikant höhere Formfehler bei den Kronen aus Korrekturtechnik / Nacera / Labor B im Vergleich zu Doppelmischtechnik / LAVA und Doceram / Labor B sowie zu Korrekturtechnik / LAVA / Labor B.

Insgesamt wurden bei den Kronen auf der Basis der Korrekturtechnik höhere Formfehler gemessen als bei den Kronen auf Basis der Doppelmischtechnik.

In dem folgenden Diagramm (Abb.19) sind die Ergebnisse für die einzelnen Abformtechniken im Vergleich zueinander dargestellt. 


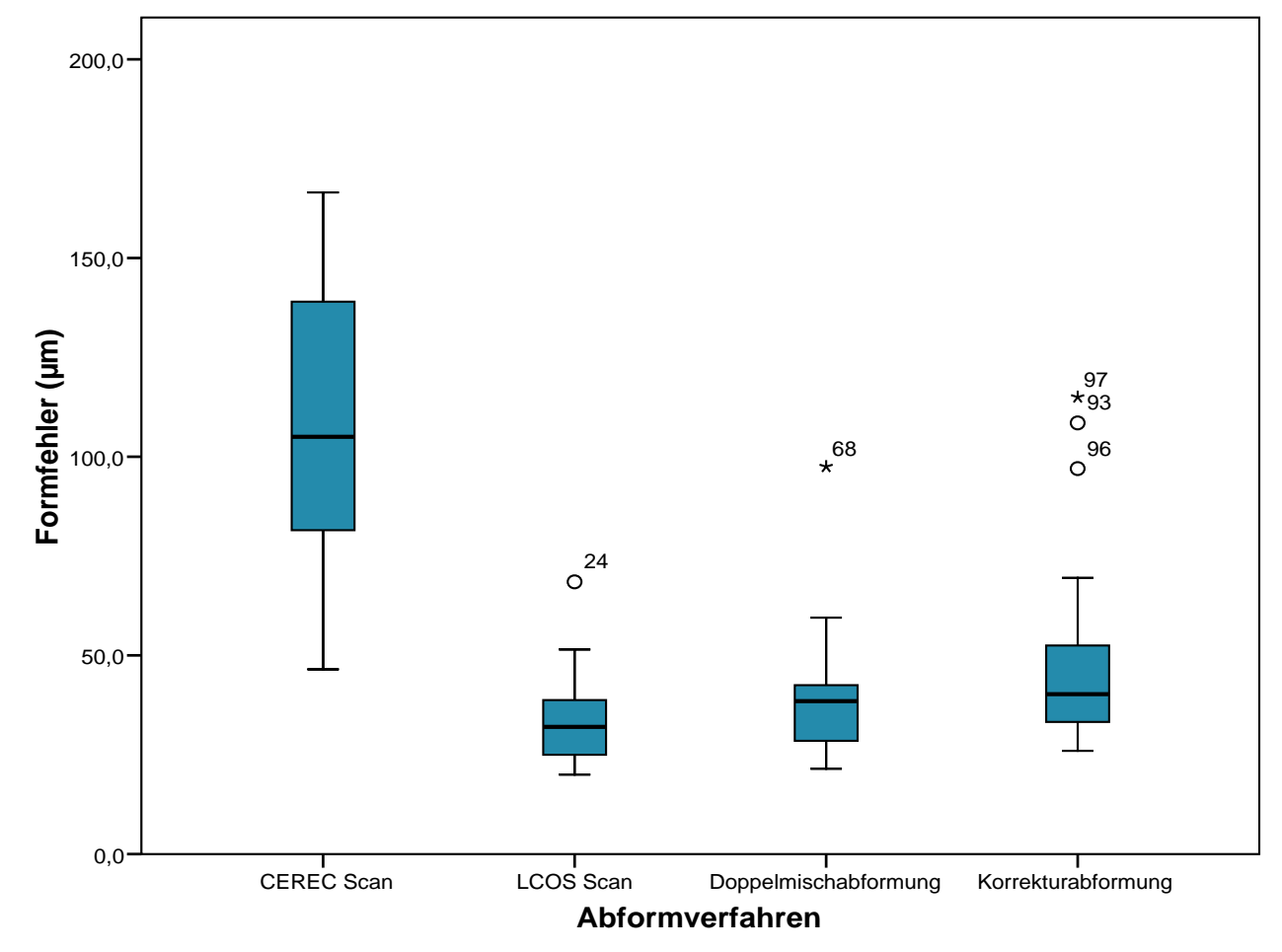

Abb.19: Boxplotdiagramm des Formfehlers bezogen auf die unterschiedlichen Abformverfahren.

Das Material LAVA wurde bei der Ermittlung des Formfehlers in drei verschiedenen Abformverfahren bei sonst gleichen Bedingungen verglichen. Wie der Tabelle 11 entnommen werden kann, ergaben sich keine signifikanten Unterschiede. Die geringsten Formfehler wiesen die Kronen aus den LCOS-Scans auf. 
Tabelle 11: Formfehler von LAVA Zirkonkronen bei unterschiedlichen Abformtechniken

\begin{tabular}{|l|l|c|c|}
\hline \multicolumn{5}{|c|}{ Mehrfachvergleich des Formfehlers (Games Howell- Test) } \\
Abhängige Variable: Formfehler $(\boldsymbol{\mu m})$ \\
\hline Vergleichsgruppe 1 & Vergleichsgruppe2 & Differenz (1-2) & Signifikanzniveau \\
\hline LCOS Scan/ LAVA/ LaborB & Doppelmischt./ LAVA/ LaborB & -8 & $\begin{array}{r}\text { nicht signifikant } \\
(\mathrm{p}=0,309)\end{array}$ \\
\hline LCOS Scan/ LAVA/ Labor B & Korrekturt./ LAVA/ Labor B. & -7 & $\begin{array}{c}\text { nicht signifikant } \\
(\mathrm{p}=0,670)\end{array}$ \\
\hline Doppelmischt// LAVA/ Labor B & Korrekturt./ LAVA/ Labor B & 1 & $\begin{array}{c}\text { nicht signifikant } \\
(\mathrm{p}=1,000)\end{array}$ \\
\hline
\end{tabular}

\subsubsection{Absolute marginale Diskrepanz}

Beim Vergleich der erhobenen Messdaten für die absolute marginale Diskrepanz im Bezug auf die angewendeten Abformtechniken ergaben sich folgende Zusammenhänge.

Bei den Kronen aus CEREC-Scan / Vita MarkII, die im Eigenlabor unserer Poliklinik hergestellt wurden und den Kronen aus CEREC-Scan / Empress CAD / Labor C ergaben sich jeweils signifikant geringere $(\mathrm{p}<0,05)$ marginale Diskrepanzen im Vergleich zu den Kronen aus den Korrekturabformungen EMF / Labor A, Gold / Labor B und LAVA / Labor B.

Zwischen den beiden Gruppen der auf CEREC-Scans basierenden Kronen ergaben sich keine signifikanten Unterschiede, die marginalen Diskrepanzen der CEREC-Scan / Vita MarkII Kronen aus dem Eigenlabor waren jedoch geringer als die der Kronen aus CEREC-Scan / Empress CAD / Labor C.

Die Kronen aus LCOS-Scan / LAVA / Labor B wiesen signifikant höhere $(p<0,05)$ Messwerte auf, als die Kronen aus Doppelmischtechnik / Doceram / Labor B. Die Messwerte der Kro- 
nen LCOS-Scan / LAVA / Labor B waren signifikant geringer $(\mathrm{p}<0,05)$ als die der Kronen aus den Korrekturabformungen Gold / Labor B und LAVA / Labor B.

Im Vergleich der beiden Gruppen der LCOS-Scan-Kronen ergaben sich geringere Werte für LCOS-Scan / LAVA / Labor C. Diese waren allerdings nicht signifikant.

Die LCOS-Scan-Kronen wiesen insgesamt nur gering erhöhte, nicht signifikante Werte im Vergleich zu den CEREC-Scan-Kronen auf.

Zwischen den Messdaten der Kronen aus der Doppelmisch- und der Korrekturtechnik bestanden teilweise signifikante Unterschiede. Die meisten, signifikant geringeren marginalen Diskrepanzen zeigten sich bei den Kronen aus Doppelmischtechnik / Nacera / Labor B gegenüber Kronen aus Korrekturtechnik $(\mathrm{p}<0,05)$. Zur besseren Übersicht werden diese Ergebnisse im folgenden Boxplot-Diagramm (Abb.20) noch einmal dargestellt.

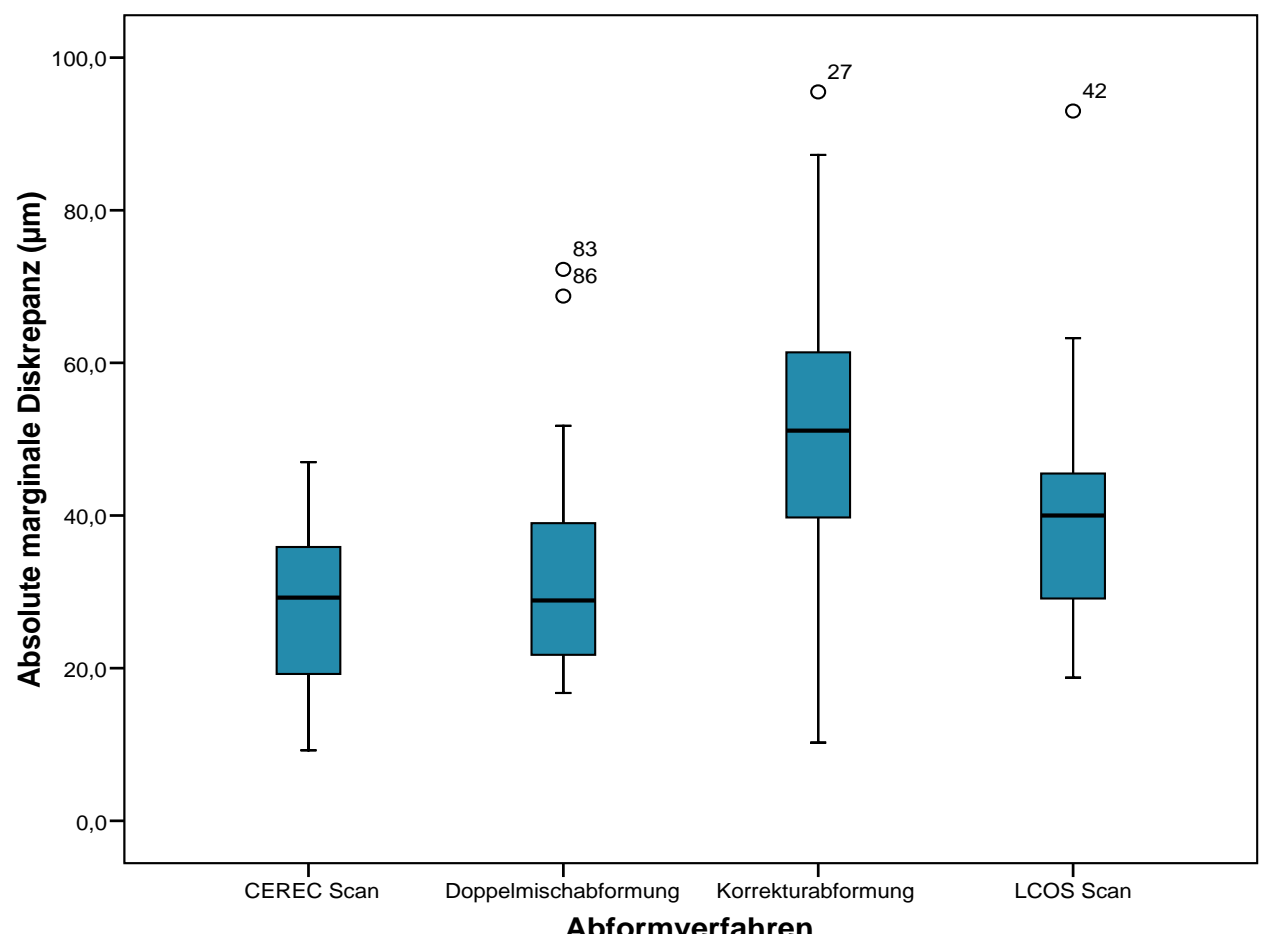

Abb.20: Boxplotdiagramm der absoluten marginalen Diskrepanz bezogen auf die unterschiedlichen Abformverfahren. 
Bei der Analyse der Messdaten wurde das Material LAVA, wie auch schon bei der Auswertung der Formfehler, in drei verschiedenen Abformverfahren verglichen. Alle Kronen wurden von demselben Dentallabor hergestellt, so dass in diesem Fall ein rein verfahrensabhängiger Vergleich möglich war. Wie in der folgenden Tabelle 12 dargestellt, wiesen die Kronen aus den Doppelmischabformungen die geringsten Werte auf. Es bestand in dieser beschränkten Betrachtung ein signifikanter Unterschied $(\mathrm{p}<0,05)$ zwischen den Kronen aus Doppelmischund Korrekturtechnik, wobei die Kronen aus den Doppelmischabformungen die geringeren marginalen Diskrepanzen hervorbrachten.

Tabelle 12: Absolute marginale Diskrepanz von LAVA Zirkonkronen bei unterschiedlichen Abformtechniken

\begin{tabular}{|l|l|c|c|}
\hline \multicolumn{5}{|c|}{ Mehrfachvergleich der absoluten marginalen Diskrepanz (Games Howell- Test) } \\
Abhängige Variable: absolute marginale Diskrepanz( $\boldsymbol{\mu m})$ \\
\hline Vergleichsgruppe 1 & Vergleichsgruppe2 & Differenz (1-2) & Signifikanzniveau \\
\hline LCOS Scan/ LAVA/ Labor B & Doppelmischt./ LAVA/ LaborB & 15 & $\begin{array}{c}\text { nicht signifikant } \\
(\mathrm{p}=0,510)\end{array}$ \\
\hline LCOS Scan/ LAVA/ Labor B & Korrekturt./ LAVA/ Labor B & -12 & $\begin{array}{r}\text { nicht signifikant } \\
\text { ( } \mathrm{p}=0,920)\end{array}$ \\
\hline Doppelmischt./ LAVA/ Labor B & Korrekturt./ LAVA/ Labor B & -27 & $\begin{array}{c}\text { signifikant } \\
(\mathrm{p}<0,05)\end{array}$ \\
\hline
\end{tabular}




\subsection{Ergebnisse des Vergleiches der verwendeten Materialien}

Betrachtet man die ausgewerteten Daten nach dem Gesichtspunkt der unterschiedlichen verwendeten Restaurationswerkstoffe, ergeben sich die in den folgenden Tabellen (13 und 14) dargestellten Ergebnisse. Vergleichbare Gruppen stellen in diesem Zusammenhang die im Dentallabor B hergestellten Kronen aus drei verschiedenen Materialien, basierend auf je zehn Korrektur- und Doppelmischabformungen dar.

In der Gruppe der Korrekturabformungen ergaben sich ein signifikant größerer $(p<0,001)$ Formfehler bei dem Material Nacera Zirkon im Vergleich zu dem Material LAVA Zirkon. In der Gruppe der Doppelmischabformungen wurden keine signifikanten Unterschiede ermittelt.

Tabelle 13: Darstellung der verschiedenen Materialen im Vergleich bei sonst gleichen Parametern (Korrekturtechnik)

\begin{tabular}{|l|l|c|c|}
\hline \multicolumn{4}{|c|}{$\begin{array}{c}\text { Mehrfachvergleich des Formfehlers (Games Howell- Test) } \\
\text { Abhängige Variable: Formfehler }(\boldsymbol{\mu m})\end{array}$} \\
\hline Vergleichsgruppe1 & Vergleichsgruppe2 & Differenz (1-2) & Signifikanzniveau \\
\hline Korrekturt./ Nacera/ Labor B & Korrekturt./ Gold/ Labor B & -2 & $\begin{array}{r}\text { nicht signifikant } \\
(\mathrm{p}=1,000)\end{array}$ \\
& Korrekturt./ LAVA/ Labor B & 19 & $\begin{array}{c}\text { signifikant } \\
(\mathrm{p}<0,001)\end{array}$ \\
\hline
\end{tabular}

Tabelle 14: Darstellung der verschiedenen Materialen im Vergleich bei sonst gleichen Parametern (Doppelmischtechnik)

\begin{tabular}{|l|l|c|c|}
\hline \multicolumn{5}{|c|}{ Mehrfachvergleich des Formfehlers (Games Howell- Test) } \\
Abhängige Variable: Formfehler $(\boldsymbol{\mu m})$
\end{tabular}


Bei der Betrachtung der absoluten marginalen Diskrepanz wiesen die Kronen aus dem Material Nacera Zirkon, sowohl auf Basis der Korrektur- als auch auf der der Doppelmischabformungen die geringsten Werte auf. Darauf folgten die Materialien LAVA Zirkon und Gold (Tabelle 15 und 16). Signifikante Unterschiede bestanden nicht.

Tabelle 15: Darstellung der verschiedenen Materialien im Vergleich bei sonst gleichen Parametern (Korrekturtechnik)

\begin{tabular}{|l|l|c|c|}
\hline \multicolumn{5}{|c|}{ Mehrfachvergleich der absoluten marginalen Diskrepanz (Games Howell- Test) } \\
Abhängige Variable: Absolute marginale Diskrepanz $(\boldsymbol{\mu m})$ \\
\hline Vergleichsgruppe1 & Vergleichsgruppe2 & Differenz (1-2) & Signifikanzniveau \\
\hline Korrekturt./ Nacera/ Labor B & Korrekturt./ Gold/ Labor B & -22 & $\begin{array}{r}\text { nicht signifikant } \\
(\mathrm{p}=0,271)\end{array}$ \\
& Korrekturt./ LAVA/ Labor B & -14 & $\begin{array}{r}\text { nicht signifikant } \\
(\mathrm{p}=0,561)\end{array}$ \\
\hline
\end{tabular}

Tabelle 16: Darstellung der verschiedenen Materialien im Vergleich bei sonst gleichen Parametern (Doppelmischtechnik)

\begin{tabular}{|l|l|c|c|}
\hline \multicolumn{4}{|c|}{ Mehrfachvergleich der absoluten marginalen Diskrepanz (Games Howell- Test) } \\
Abhängige Variable: absolute marginale Diskrepanz $(\boldsymbol{\mu m})$ \\
\hline Vergleichsgruppe1 & Vergleichsgruppe2 & Differenz (1-2) & Signifikanzniveau \\
\hline Doppelmischt./ Nacera/ Labor B & Doppelmischt// Gold/ Labor B & -21 & $\begin{array}{r}\text { nicht signifikant } \\
(\mathrm{p}=0,090)\end{array}$ \\
& Dppelmischt./ Lava/ Labor B & -11 & $\begin{array}{r}\text { nicht signifikant } \\
(\mathrm{p}=0,058)\end{array}$ \\
\hline
\end{tabular}




\subsection{Ergebnisse des Vergleiches der verschiedenen zahntechnischen Labore.}

Die Datensätze der LCOS-Scans wurden im Vorfeld zur Herstellung der Prüfkörper an zwei unterschiedliche Dentallaboratorien versendet, um einen konkreten Vergleich der durch die Labore durchgeführten Arbeit anstellen zu können. Beide Laboratorien verwendeten zur Herstellung LAVA Zirkonoxid.

Die Kronen aus dem Labor B $(48 \mu \mathrm{m})$ wiesen im Gegensatz zu den Kronen aus dem Labor C $(33 \mu \mathrm{m})$ höhere, allerdings nicht signifikante Werte bei der Messung der absoluten marginalen Diskrepanz auf. Der Formfehler der LAVA-Kronen aus dem Dentallabor B $(29 \mu \mathrm{m})$ war hin-

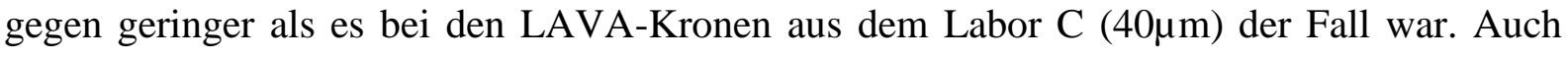
hier waren die Unterschiede jedoch nicht signifikant.

Auch bei den konventionell abgeformten Kronen konnte ein Vergleich zwischen zwei Laboratorien angestellt werden. Von dem Labor A und dem Labor B wurden jeweils Kronen aus Nacera Zirkonoxid und einer goldhaltigen Legierung auf der Basis von Korrekturabformungen hergestellt. Hierbei ergaben die Messungen in keinem Fall signifikante Unterschiede.

\subsection{Zusammenfassung der Ergebnisse}

Die vielfältigen Ergebnisse lassen sich im Hinblick auf die im Ziel dieser Arbeit formulierten Fragen wie folgt zusammenfassen:

Die mit den geprüften intraoralen Scannern gefertigten Restaurationen waren hinsichtlich ihrer Passgenauigkeit (sowohl marginale Diskrepanz als auch innere Rundheit) mit den Ergebnissen der konventionellen Abformung vergleichbar.

Ein signifikanter Einfluss der verwendeten Restaurationswerkstoffe und beauftragten Laboratorien konnte nicht festgestellt werden.

Damit konnten beide Nullhypothesen nicht verworfen werden. 


\section{Diskussion}

\subsection{Diskussion der verwendeten Materialien und Methoden}

\subsubsection{Urmodell}

Bei der Betrachtung des für diese Studie angefertigten Urmodells, insbesondere des Urstumpfes ist zu sagen, dass dieser durch seine Geometrie deutlich von den realen Bedingungen, die bei einem präparierten Zahn gegeben sind, abweicht. Die parallelen Flächen des Stumpfes waren aus messtechnischen Gründen erforderlich und führten somit dazu, dass die angewendeten Techniken von der Abformung bis hin zur Anfertigung der Kronen erschwert waren. Diese Extremsituation machte es außerdem möglich, die Grenzen der untersuchten Verfahren bewusst aufzuzeigen. Kleine Fehler im Ablauf der Arbeitsketten konnten somit sehr leicht ermittelt werden. Ein Beispiel dafür ist, dass jede einzelne Krone vor der Bestimmung der absoluten marginalen Diskrepanz mit einem Diamanten auf den Urstumpf aufgepasst werden musste, da die parallelen Flächen des Stumpfes und auch der angefertigten Kronen eine komplette Repositionierung so nicht ermöglichten. Dieser Arbeitsschritt musste per Hand durchgeführt werden und konnte daher dazu führen, dass die Kronen vielleicht nicht komplett in Sollposition gebracht werden konnten, was im Anschluss eventuell fehlerhafte Messungen der Randspalten zur Folge hatte. Auch bei der Bestimmung des Formfehlers wurde deutlich, dass die Stumpfgeometrie sich als Herausforderung, besonders für die verwendeten Fräseinheiten darstellte, da es in diesem Bereich mitunter große Abweichungen gab. Auf diese, im Zusammenhang mit der Vermessung der Kronen aufgetretenen Probleme wird im späteren Verlauf noch näher eingegangen.

Die Methode, Untersuchungen anhand eines Stahlurmodells durchzuführen, war nicht neu. Zahlreiche andere Autoren verwendeten in ihren Studien ebenfalls Metallstümpfe und Modelle 20, 27, 78 . Bereits 1978 führten Lehmann und Burgdorf eine Studie durch, in der sie einen genormten Stahlstumpf in Korrekturtechnik abformten und die resultierenden Gipsstümpfe mit dem Urstumpf verglichen ${ }^{49}$. Bader und Setz bestimmten 1991 die Wiedergabegenauigkeit 
von Abformmaterialien ebenfalls in einem ähnlichen Verfahren ${ }^{3}$, genau wie Nissan et al. im Jahr $2000{ }^{58}$. Tinschert et al. fertigten 2001 ebenfalls ein Urmodell aus Stahl zur Bestimmung marginaler Diskrepanzen an, bei dem die jeweiligen Urstümpfe mit einer zirkulären Hohlkehlpräparation, ähnlich wie in der hier vorgestellten Studie, präpariert waren ${ }^{73}$. Die Hohlkehlpräparation war bei dieser Untersuchung, bei der eine Präparation zur Herstellung von Zahnersatz gescannt werden musste, die Technik der ersten Wahl. Der angewendete Scanner, egal ob Intraoral- oder Extraoralscanner, kann abgerundete Kanten wesentlich besser erkennen und umsetzen. Bei Stufen und nicht gerundeten Kanten kommt es erfahrungsgemäß häufiger zu Fehlern im Scannprozess, da dort auch unterschnittige Bereiche entstehen können, die die Kamera eventuell nicht komplett einsehen kann.

\subsubsection{Durchführung der Abformungen und Herstellung der Prüfkörper}

Bei den konventionellen Abformverfahren wurden die für die Herstellung von festsitzendem Zahnersatz gängigen Methoden, die Korrektur- und Doppelmischtechnik ausgewählt. Diese wurden, wie schon beschrieben, bereits für zahlreiche Untersuchungen herangezogen. Die Abformungen wurden aus Gründen einer möglichst praxisnahen Durchführung manuell durch eine approbierte Zahnärztin durchgeführt. In anderen Studien wurden die Abformungen meist maschinell vorgenommen, wie beispielsweise bei Wichmann, Borchers und Limmroth, die eine Universalprüfmaschine verwendeten ${ }^{78}$. Auch Fenske et al. verwendeten eine Abformmaschine ${ }^{20}$. Der Vorteil dieser Verfahren lag natürlich in einer reproduzierbareren Qualität der jeweiligen Abformungen, da immer unter einem vorher genau eingestellten Anpressdruck gearbeitet werden konnte. Fehler in den Abformungen durch eventuell ungleichmäßige Schichtstärken der verschiedenen Materialien waren so sicherlich vermeidbarer.

In der vorliegenden Studie wurde das Urmodell zunächst 30mal mittels der Korrekturtechnik abgeformt und es wurden 30 Modelle und darauf 30 Kronen aus drei verschiedenen Materialien $(n=10)$ gefertigt. Um im Bezug auf die Korrekturtechnik Vergleichsdaten zu erhalten wurden weitere 10 Abformungen genommen und an das Labor B versendet. Diese fertigten wiederum 30 Kronen aus 3 verschiedenen Materialien an. Um im weiteren Studienverlauf 
einen vergleichbaren Restaurationswerkstoff für die Kronen aus den Scan-Daten des LCOS zu erhalten, wurde nun LAVA Zirkonoxid anstelle der von Labor A gelieferten EMFLegierung verwendet. Als weitere konventionelle Vergleichstechnik wurde die Doppelmischabformung gewählt und es wurden weitere 10 Abformungen an das Labor B gesendet, mit denen in gleicher Weise wie auch mit den 10 Korrekturabformungen verfahren wurde. Nun war bei den aus dem Labor B gelieferten Kronen nicht für jede ein Arbeitsmodell vorhanden. Da die Kronen aus Nacera und LAVA Zirkonoxid aber aus den vom Gipsmodell im Dentallabor gescannten Daten gefräst wurden und nicht auf den Modellen selbst angefertigt werden mussten, waren dadurch keine Nachteile für die Ergebnisse der Studie zu befürchten. Die Kronen wurden bei der späteren Auswertung alle nur auf dem Urstumpf und nicht zusätzlich auf den Arbeitsmodellen vermessen.

Die Durchführung der Scans wurde wie auch schon die Abformungen von einem eigens darauf geschulten, approbierten Zahnarzt durchgeführt. Auch hier wurde bewusst eine manuelle Durchführung gewählt. Das Urmodell oder die Kamera wurden dafür in keine spezielle Halterung eingebaut, so wie es durch Luthardt in einer Studie mittels eines Stativs für die Kamera beschrieben wurde ${ }^{51}$, sondern auf einer stabilen Unterlage positioniert. Eine Fixierung der Kamera in einem definierten Abstand zum Urmodell wäre zu überdenken, um Ungenauigkeiten im Scanprozess, die durch den Anwender bedingt sind zu minimieren. Zur Simulation einer praxisnaheren Situation während des Scanvorganges wäre es denkbar gewesen, das Urmodell zusätzlich in einen Phantomkopf einzuspannen, wie es Da Costa 2010 in einer Studie mit dem CEREC 3D Scanner durchführte ${ }^{11}$.

Im Verlauf unserer Studie wurden 10 Scans mit LCOS durchgeführt und zum Vergleich an zwei verschiedene Laboratorien $(B, C)$ gesendet. Mit der CEREC-Bluecam wurden zunächst 10 Scans durchgeführt, die wiederum an eines der Labore (C) versendet wurden. Auch in diesem Fall wäre ein direkter Vergleich zwischen den Laboratorien wünschenswert gewesen, jedoch verfügte das zweite Labor nicht über eine CEREC-Fräseinheit. Um dennoch einen Vergleich zu erhalten, wurden 10 weitere Scans mit der CEREC-Bluecam durchgeführt und daraus 10 Kronen auf der in unserer Poliklinik vorhandenen Fräseinheit hergestellt. So konnte eine Vergleichsgruppe gewonnen werden, die sehr praxisnah einzuschätzen ist, da nicht wie in den anderen Fällen eine Laborfräse verwendet wurde und der Anwender, wie in vielen Praxen ein Zahnarzt und kein speziell ausgebildeter Zahntechniker war. 


\subsubsection{Diskussion der Untersuchungsparameter}

\subsubsection{Rundheit: Bestimmung des Formfehlers}

Zur Ermittlung der Wiedergabegenauigkeit von Abformverfahren wurden in verschiedenen Arbeiten viele unterschiedliche Möglichkeiten beschrieben. Die Wahl des Verfahrens hing dabei vor allem davon ab, unter welchem Gesichtspunkt der Untersucher diese Wiedergabegenauigkeit betrachten wollte. In zahlreichen Studien beschäftigten sich diverse Autoren damit, die Dimensionsveränderungen von aus verschiedenen Abformungen resultierenden Gipsstümpfen zu vergleichen, um daraus vor allem eine Aussage über material- und auch verfahrensspezifische Eigenheiten treffen zu können ${ }^{31,49,58,83}$. In der hier präsentierten Studie interessierte allerdings, wie genau die innere Geometrie der Prüfkörper mit der Geometrie des zuvor vermessenen Urstumpfes übereinstimmte. Dies wurde mit Hilfe einer 3DKoordinatenmessmaschine ermittelt. Diese mechanische Antastung von Werkstücken wurde auch 1990 von Wichmann, Borchers und Limmroth in einem ähnlichen Versuchsaufbau durchgeführt. Auch sie formten ein Urmodell unter anderem in Korrektur- und Doppelmischtechnik ab. Im Anschluss wurden hier die resultierenden Gipsstümpfe mit dem Urstumpf verglichen. Der verwendete Taster hatte ebenfalls einen Durchmesser von $1 \mathrm{~mm}^{78}$. Auch Fenske et al. verwendeten in ihrer Untersuchung zur Dimensionstreue von Modellstümpfen eine 3DKoordinatenmessmaschine mit der sie die Modelle mit jeweils etwa 300 Einzelmesspunkten zirkulär abtasteten ${ }^{20}$. Um eine möglichst detaillierte Aussage über die innere Passung der Prüfkörper treffen zu können, und so den resultierenden Formfehler möglichst genau zu bestimmen, wurde in der vorliegenden Studie bei der Wahl der Einzelmesspunkte eine höhere Anzahl von 10 x 50 Punkten pro Kronenlumen gewählt. Um mögliche Fehler bei der Abtastung durch das zu starke Erfassen von herstellungsbedingten Schleifspuren oder Ähnlichem zu minimieren, wurde in der aktuellen Studie nicht der kleinst mögliche Taster, sondern der Taster mit 1mm Durchmesser gewählt, wie auch schon bei Wichmann, Borchers und Limmroth. Andere Autoren ermittelten die interne Passung von Kronen anhand eines Silikonfilmes, welcher durch ein (vor dem Aufsetzen der Kronen auf den jeweiligen Stumpf) in das Lumen appliziertes, dünnfließendes Silikon entstand, dass nach der Aushärtung entnommen und dessen Dicke dann meist unter Mikroskopen vermessen wurde ${ }^{10,47}$. Bei dieser Technik sind natürlich materialspezifische, werkstoffkundliche Effekte bei der Polymerisation des Silikons zu beachten, die eventuell zu Ungenauigkeiten führen können. Eine andere beschrie- 
bene Methode war die Betrachtung unter einem Rasterelektronenmikroskop oder einem Auflichtmikroskop. Hierfür wurden die zu vermessenden Kronen meist auf den jeweiligen Stumpf zementiert, um im Anschluss präpariert werden zu können ${ }^{6,25,68}$. Diese Technik war mit einem einzelnen Urstumpf jedoch nicht möglich. Außerdem erhielt man dabei auch immer nur eine Aussage über einen Teilbereich der internen Passung, da nicht das komplette Lumen erfasst werden konnte.

\subsubsection{Absolute marginale Diskrepanz}

Bei der Messung der absoluten marginalen Diskrepanz wurde die Methode, den Randbereich der Kronen auf dem jeweiligen Urstumpf direkt zu betrachten gewählt, um bei einer entsprechenden Vergrößerung die Diskrepanzen mittels Mikrometeruhren direkt ablesen zu können. Mit diesem oder ähnlichen Verfahren arbeiteten bereits zahlreiche Untersucher. Da Costa bestimmte die Größe der Randspalten in seiner Untersuchung ebenfalls optisch, bei 50facher Vergrößerung. Er ermittelte Messwerte von 12 Punkten je Restauration ${ }^{11}$. Auch Lee et al. betrachteten die Randspalten in ihrer Studie auf einem Urstumpf unter einem Messmikroskop. Sie wählten 50 Punkte bei 100 facher Vergrößerung ${ }^{48}$. Sulaiman et al. sowie Weaver, Johnson und Bales nahmen die Messungen auch auf dem Urstumpf in ähnlicher Weise vor ${ }^{71,76}$. Hier wurden, wie in dieser Studie, vier Messwerte pro Krone erhoben. Eine andere Möglichkeit zur Bestimmung der marginalen Passform war, wie auch schon zur Bestimmung der internen Passung, das Auffüllen der Kronen mit einem dünnfließenden Silikon vor der Reposition auf den Stumpf oder Zahn. Dieser entstandene Film wurde dann, wie bereits zuvor schon beschrieben, häufig mit einem Mikroskop vermessen ${ }^{7,10,22,47}$. Auch mit Hilfe eines Elektronenmikroskops kann die Randpassung von Kronen zirkulär beurteilt werden, wie es Bindl 1999 beschrieb ${ }^{6}$. Die Methode nach der in der vorliegenden Studie die absolute marginale Diskrepanz bestimmt wurde stützt sich auf die von Holmes 1989 veröffentlichte Definition ${ }^{32}$. In vielen bislang veröffentlichten Studien wurde schon nach diesem Prinzip die marginale Passform ermittelt, somit kann dies als etablierte Methode angesehen werden 7, 40, 44, 73, 74, 79 . Um ein noch aussagekräftigeres Ergebnis im Bezug auf die absolute marginale Diskrepanz zu erhalten, wäre eine Messung an mehreren einzelnen Stellen, wie etwa in der oben beschriebenen Unter- 
suchung von Da Costa und Lee, denkbar. Auch könnte ein zweites Verfahren hinzugezogen werden. Es wäre zum Beispiel denkbar gewesen, zusätzlich ein Replika aus Silikon herzustellen und dieses zu vermessen, wie es die weiter oben im Text bereits genannten Autoren beschrieben hatten.

\subsubsection{Statistische Auswertung}

In der Kombination aller drei Faktoren (Material, Labor, Abformverfahren) waren durchweg gleich große Gruppen der verschiedenen Prüfkörper $(n=10)$ gegeben und es lag, bei einem Vergleich auf dieser Ebene ein balanciertes Datendesign vor.

Bei allen Auswertungen lag das Hauptaugenmerk auf den jeweiligen Mittelwerten der Messwerte. Im Gegensatz zu einer Darstellung der Extremwerte konnte eine Aussage darüber getroffen werden, welche durchschnittlichen Ergebnisse bei der Genauigkeit der Verfahren zu erwarten waren. Inwieweit diese Mittelwerte im Verhältnis zu ihren Extremen einzuschätzen waren, konnte der jeweiligen ermittelten Standardabweichung entnommen werden. Dass eine gute Standardisierbarkeit der durchgeführten Verfahren realisiert wurde, zeigte sich anhand der geringen resultierenden Standardabweichung. Im Anschluss an den einfachen Gruppenvergleich wurde eine Varianzanalyse durchgeführt um herauszufinden, ob die gefundenen Unterschiede hinreichend ausgeprägt waren und um so eine Verallgemeinerung über die Stichprobe hinaus zu erlauben. Mit dem Levene-Test wurde eine Ungleichheit der Varianzen ermittelt, so dass daraufhin der Welch- und Brown-Forsythe-Test zum Einsatz kamen. Diese ergaben, dass hochsignifikante Unterschiede in den Vergleichsgruppen vorlagen. Welche Gruppen sich genau unterschieden, wurde mit dem Games-Howel-Test überprüft. Bei gleichen Varianzen wäre als Post-Hoc-Test der Tukey-Test angewendet worden ${ }^{64}$. 


\subsection{Diskussion der Ergebnisse}

\subsubsection{Formfehler}

Bei den Ergebnissen des Formfehlers der, wie zuvor schon erwähnt, die interne Passform der Kronen im Bezug auf den Urstumpf wieder gab, bestanden zum Teil geringe und auch sehr hohe Werte. Besonders bei den Prüfkörpern, die aus CEREC-Scans resultierten gab es große Abweichungen, im Gegensatz zu den LCOS-Kronen. Die Kronen auf Basis der CERECScans vielen deutlich durch ihre signifikant höheren Werte im Vergleich zu allen anderen Kronen auf. Auch andere Studien in denen mit dem CEREC-System gefräste Kronen untersucht wurden lieferten ähnlich hohe und meist sogar noch höhere Abweichungen. Besonders in Verbindung mit dem Werkstoff Vita Mark II, den wir für eine Serie ebenfalls verwendeten, waren die gemessenen Werte auch bei den anderen Autoren ähnlich hoch und die Werte der im Vergleich gemessenen Kronen deutlich, meist signifikant niedriger. Lee erhob im Mittel

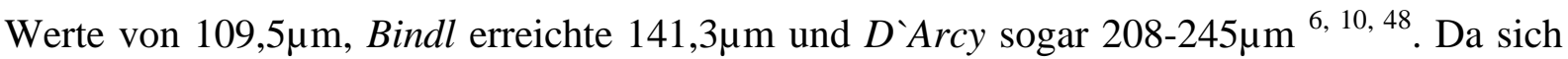
all diese, von den verschiedensten Autoren erhobenen Werte in einer ähnlichen Größenordnung wie in der aktuell präsentierten Studie befanden, war es als unwahrscheinlich anzusehen, dass es ausschließlich durch Messfehler zu diesen Ergebnissen kam. Auch fiel bei der hier dargestellten Ergebnisauswertung eine sehr hohe Streuung der Werte auf. Eine Erklärung für diese gemessenen Werte im Fall der Vita Mark II Kronen wurde bereits in der Anwendung der sich im Eigenlabor der Poliklinik für Zahnärztliche Prothetik in Giessen befindlichen CEREC-Fräseinheit gesehen. Unter Umständen würden CEREC-Laborfräsen doch ein genaueres Ergebnis liefern. Auch ist der Zahntechniker sicherlich geübter im Umgang mit den Einstellungen vor dem Fräsvorgang. Da im Eigenlabor mit den im System vorgegebenen Einstellungen für die Zementspaltbreite gearbeitet wurde, war dies eventuell ein weiterer Faktor, der unter anderem zu diesen hohen Messdaten führte. Im direkten Vergleich zwischen den Ergebnissen der CEREC-Kronen, die im Labor $\mathrm{C}$ aus Empress CAD hergestellt wurden mit den im Eigenlabor (D) gefrästen, ergaben sich zwar für die ersteren geringere Formfehler, jedoch lagen beide Serien deutlich über den Werten auch der anderen gefrästen Kronen. In der Bewertung dieser Ergebnisse wurde also davon ausgegangen, dass diese Ungenauigkeiten wahrscheinlich durch den Fräsvorgang selbst bedingt waren und zusätzlich durch Einstellungsparameter beeinflusst wurden. Auch der Scanvorgang spielte im Bezug auf die Wiedergabege- 
nauigkeit eine Rolle. So ermittelte Luthard 2005 im Laborversuch für den CEREC 3D Scan-

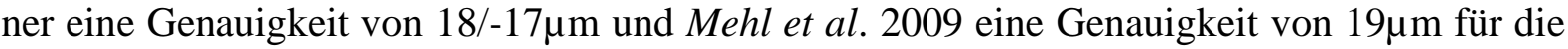
CEREC-Bluecam. Die Gründe für diese Ungenauigkeiten erklärten sich unter anderem durch kohärentes Rauschen, welches in Abhängigkeit vom Abstand der Kamera im Bezug auf das zu scannende Objekt steht. Diese zufällig entstehenden Messfehler werden von der Software automatisch gefiltert, was negative Auswirkungen auf die Genauigkeit hat. Die neue Linsenanordnung in der CEREC-Bluecam bedingt eine bessere Wiedergabegenauigkeit durch weniger notwendige Filterung. Auch das nun anstelle des bislang genutzten Infrarotlichtes ( $\alpha=820 \mathrm{~nm})$ verwendete blaue Licht, mit einer geringeren Wellenlänge ( $\alpha=470 \mathrm{~nm})$, verbesserte die Auflösung und die Genauigkeit ${ }^{51,56}$. Die Kronen, die auf Basis der Scans mit dem LCOS hergestellt wurden, lieferten in der hier präsentierten Untersuchung die geringsten Werte aller Proben bei der Messung des Formfehlers. Dieses Ergebnis ließ vermuten, dass die Scan Technik im LCOS möglicherweise genauer war. Leider fehlen Vergleichsdaten aus anderen Studien bezüglich der internen Geometrie und Wiedergabegenauigkeit von LCOS.

Die Resultate der auf herkömmliche Weise abgeformten Kronen fielen wesentlich homogener aus. Die gemessenen Formfehler lagen zwischen 35 und $56 \mu \mathrm{m}$. Die Kronen auf Basis der Korrekturabformung zeigten höhere Abweichungen als die Kronen aus den Doppelmischabformungen. Ein Ergebnis, welches auch so zu erwarten war, da mittlerweile in zahlreichen Studien zum Thema Wiedergabegenauigkeit von Abformmaterialien belegt wurde, dass es bei der Anwendung der Korrekturtechnik zu höheren Abweichungen als bei der Doppelmischtechnik kommt ${ }^{3,31,49,50}$. Wichmann, Borchers und Limmroth stellten bei einer Untersuchung zu diesem Thema 1990 fest, dass es bei Korrekturabformungen im Mittel zu einer Verkleine-

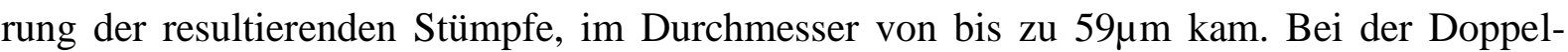
mischabformung resultierten dagegen eher vergrößerte Stümpfe $(15-42 \mu \mathrm{m}){ }^{78}$. Diese Angaben waren vergleichbar mit den in dieser Studie gemessenen Werten.

Insgesamt stellt sich die Frage, wie relevant diese gemessenen Abweichungen für klinische Situationen sind. Um Kronen und Brücken eingliedern zu können besteht immer ein gewisser Platzbedarf zwischen Zahn und Restauration, der für den Zement benötigt wird. Dieser muss eine bestimmte Größe haben, sollte aber auch nicht zu groß sein, da der Verbund sonst beeinträchtigt würde und der Zement schneller ausgewaschen werden könnte ${ }^{38}$. Da sich dies auch wiederum auf die marginale Passform auswirken kann, wird dieser Aspekt im Verlauf wieder aufgegriffen und noch näher beleuchtet. 


\subsubsection{Absolute marginale Diskrepanz}

Bei der Bestimmung der absoluten marginalen Diskrepanzen lagen alle in der vorliegenden Studie gemessenen Werte unter $100 \mu \mathrm{m}$. Dieser Wert sollte, wie schon in Kapitel 2 beschrieben, möglichst nicht überschritten werden ${ }^{77}$. Die meisten Ergebnisse lagen im Mittel sogar unter $50 \mu \mathrm{m}$ wie es Dreyer-Jörgensen schon 1958 forderte ${ }^{13}$. Lediglich bei drei Serien von Kronen basierend auf Korrekturabformungen wurde dieser Wert überschritten (55-68 $\mu \mathrm{m})$. Im Laborversuch ist es natürlich uneingeschränkt möglich, alle Bereiche des Urstumpfes abzuformen. Klinische Faktoren, die das Ergebnis einer Abformung beeinträchtigen können, sind von Anfang an ausgeschlossen. Daher ist die Aussage über die marginale Genauigkeit in der hier präsentierten Studie in einem anderen Kontext zu sehen, als es in klinischen Studien der Fall ist. Betrachtet man im Vergleich dazu Untersuchungen, die in vivo durchgeführt wurden, stiegen die gemessenen Werte der marginalen Diskrepanzen meist erheblich an ${ }^{54,68,79}$. In der 2010 veröffentlichten, klinischen Untersuchung von Syrek et al. wurden ähnliche Werte für die marginale Diskrepanz von Kronen, sowohl basierend auf Korrekturabformungen $(71 \mu \mathrm{m})$, als auch auf LCOS Scans $(49 \mu \mathrm{m})$ ermittelt ${ }^{72}$. Die in dieser Studie gemessenen Werte betrugen für LCOS 33-48 $\mu \mathrm{m}$. Dies zeigt, dass die Genauigkeit, die wir im Laborversuch, bezogen auf die intraoralen Scanner erreicht haben, auch am Patienten möglich ist. Die Anzahl der Patienten in dieser Studie war mit zwanzig allerdings relativ gering. Leider gibt es bisher keine weiteren vergleichbaren klinischen Untersuchungen, die dieses Ergebnis bestätigen könnten.

Wie in den ersten Kapiteln bereits beschrieben wurde, spielt die marginale Passform von Kronen und Brücken eine entscheidende Rolle in Bezug auf die Überlebensdauer einer Restauration besonders im Bezug auf Sekundärkaries im Kronenrandbereich und die gingivale, sowie parodontale Gesundheit ${ }^{4,26}$. Der notwendige Zementspalt für die Befestigung einer Restauration wirkt sich auch auf die Passform von Zahnersatz im besonders sensiblen Randbereich aus. Ein zu großer Zementspalt führt zu schnellerem, verstärktem Auswaschen des Zementes und die Krone wird „undicht“. Jacobs fand in einer Studie heraus, dass es bei Spaltmaßen über $100 \mu \mathrm{m}$ zu einer verstärkten Auflösung kommt ${ }^{38}$. Abhängig ist dies natürlich auch immer von der Korngröße des verwendeten Zementes. Ein weiterer Effekt, der für einen möglichst geringen Randspalt in diesem Zusammenhang spricht, ist der, dass sich Kronen nach Zementierung um einen gewissen Betrag durch den Zement aufdehnen und der Randspalt sich dadurch vergrößert. Dies zeigte auch eine Untersuchung von Weaver et al., der eine 
Vergrößerung des messbaren Spaltes von $10-20 \mu \mathrm{m}^{76}$ fand. Es wäre nun denkbar gewesen, dass die Kronen in der vorliegenden Studie, bei denen größere Formfehler gemessen wurden, auch eine größere marginale Diskrepanz aufwiesen. Dies war allerdings nicht der Fall, ganz im Gegenteil. Die Kronen aus CEREC-Scan, bei denen mit Abstand die höchsten Formfehler gemessen wurden, hatten sehr geringe marginale Diskrepanzen. Man kann also, wie bereits im letzten Absatz diskutiert, davon ausgehen, dass die Abweichungen der internen Geometrie nicht direkt mit einem größeren Zementspalt zusammen hingen, der bei der Fräsung eingestellt wurde.

Betrachtet man unter all den vorliegenden Gesichtspunkten nun einmal die Ergebnisse, so kann man von einem durchaus guten Resultat bei allen gemessenen Kronen sprechen.

\subsubsection{Diskussion der Ergebnisse der verwendeten Abformverfahren}

Das Ziel dieser Studie war es die Genauigkeit der neuen intraoralen Scannverfahren mit der von herkömmlichen Abformtechniken zu Vergleichen, um eine Aussage darüber treffen zu können, ob diese Verfahren bereits eine echte Alternative bieten können. Mit dem verwendeten Versuchsaufbau wurde bewusst eine Idealsituation simuliert, um die Untersuchung so genau wie möglich, rein auf die Ermittlung der Genauigkeit der angewendeten Verfahren zu konzentrieren. Die hier präsentierten Ergebnisse sind, besonders im Hinblick auf die marginale Genauigkeit vielversprechend. In einer Studie die Ender 2011 veröffentlichte, wurde ebenfalls die konventionelle Abformung mit den beiden auch von uns untersuchten intraoralen Scannern verglichen. Dabei wurden allerdings ganze Kiefer gescannt und im Anschluss mit einem Referenzscan mittels einer Software verglichen. Die Ergebnisse für die Wiedergabegenauigkeit lagen bei $55 \pm 21,8 \mu \mathrm{m}$ für die konventionelle Abformung, $49 \pm 14,2 \mu \mathrm{m}$ für die CEREC-Bluecam und 40,3 $\pm 14,1 \mu \mathrm{m}$ für den LCOS. In dieser Untersuchung zeigt sich, dass die digitalen Verfahren absolut vergleichbare Ergebnisse zu konventionellen Verfahren erzielten ${ }^{15}$. Auch die in vivo Studie von Syrek et al. von 2010, in der die marginalen Genauigkeiten von Kronen vermessen wurden, die einerseits auf Basis von Korrekturabformungen und andererseits auf Basis von LCOS-Scans hergestellt wurden, zeigt ähnliche Resultate. Die gemes- 
senen Werte betrugen für die Korrekturabformung im Mittel $71 \mu \mathrm{m}$ und für die LCOS-Scans $49 \mu \mathrm{m}^{72}$. In beiden Studien schnitten die digitalen Abformungen besser ab als die konventionellen. Auch bei der aktuellen Untersuchung konnten teilweise sehr gute Ergebnisse, besonders bei der marginalen Diskrepanz, erzielt werden.

Aufgrund der Ergebnisse stellt sich natürlich die Frage, wodurch diese insgesamt gute Genauigkeit der intraoralen Verfahren bedingt ist. Betrachtet man die Arbeits- und Werkstoffkette von einer klassischen Abformung bis hin zur fertigen Restauration, so stößt man auf zahlreiche Schritte, die das Ergebnis hinsichtlich der Genauigkeit stark beeinflussen können. Die Auswahl des Abformlöffels, der richtige Umgang mit den verwendeten Abformmaterialien und schließlich die Modellherstellung sind in diesem Zusammenhang entscheidend ${ }^{82}$. All diese Faktoren tragen dazu bei, dass die Standardisierung konventioneller Abformverfahren erschwert ist und bleibt. Bei den intraoralen Scannern entfällt dagegen ein Großteil dieser Faktoren. Außerdem entsteht so ein Vorteil durch Zeit- und eventuell Kostenersparnis. Nach Durchführung des Scans, kann das Ergebnis direkt am Bildschirm kontrolliert und gegebenenfalls korrigiert werden, wodurch eine weitere Standardisierung und Qualitätsverbesserung erfolgen kann. Die Daten werden dann im Anschluss direkt weiter verarbeitet ${ }^{14}$. Die Herstellung von Modellen erfolgt parallel aus dem gleichen Datensatz, der auch zur Herstellung der Restauration verwendet wird und dient nicht, wie bei den herkömmlichen Verfahren als direkte Arbeitsgrundlage ${ }^{33}$. Bei der Durchführung eines direkten Scans kann es natürlich, wie auch bei einer Abformung, zu Fehlern kommen. Ist der Behandler noch ungeübt in der Bedienung, muss der Scan eventuell durch häufigeres nachscannen vervollständigt werden, was zu ungenaueren Ergebnissen durch erhöhte Datenmengen führen kann. Allein das Zusammensetzen der gewonnenen Einzelscans zu einem Datensatz (Matching) führt, wie Rudolph et al. untersuchten, zu einem Fehler von mittelwertig $14-17 \mu \mathrm{m}^{65}$.

Im Hinblick auf die Anwendung intraoraler Scanner am Patienten muss beachtet werden, dass nur Areale wiedergegeben werden können, die auch für die Kamera optisch erfassbar sind. Das bedeutet, dass sich zwangsläufig Probleme bei stark infragingival liegenden Präparationsrändern ergeben, welche eventuell mit einer konventionellen Korrekturabformung noch erreichbar sind. Diese Situation ist allerdings im klinischen Alltag sehr häufig anzutreffen. Von Seiten der Entwickler sollte daher auch weiter daran gearbeitet werden, Möglichkeiten zur Darstellung dieser Areale zu finden. Da dies mit den momentan verfügbaren optischen Scannern nur, wie schon beschrieben, eingeschränkt möglich ist, gilt auch zu überlegen, ob eine alternative Technik zur Datenaufnahme denkbar wäre. Bereits im Jahr 2008 wurde ein auf 
Ultraschall basierender, sogenannter Mikroscanner vorgestellt. Die Hersteller berichteten, dass die Darstellung subgingivaler Bereiche problemlos möglich sei und Sulcusblutungen oder Speichelfluss zu keinen Einschränkungen führen. Ein vielversprechender Ansatz, zu dem es bislang jedoch noch keine wissenschaftlichen Studien gibt ${ }^{36}$. Auch Patienten mit geringer Mundöffnung könnten ein Problem darstellen, da mit den Scan-Handstücken Präparationen im Molarenbereich eventuell gar nicht erreicht werden können. Werden all diese Punkte beachtet, können, wie sich bereits in der klinischen Studie von Syrek et al. Zeigt, Ergebnisse erzielt werden, die in derselben Größenordnung liegen, wie die in der vorliegenden in vitro Studie ermittelten ${ }^{72}$.

Weitere umfassende Studien müssen folgen, um die Anwendbarkeit intraoraler Scanner, besonders im klinischen Alltag, als Alternative zur konventionellen Abformung zu testen. Das Potential ist als vielversprechend einzuschätzen, besonders wenn man den, im Vergleich zu konventionellen Abformtechniken, bisher erst sehr kurzen Entwicklungszeitraum betrachtet.

\subsubsection{Diskussion der Ergebnisse des Vergleiches der verwendeten Materialien}

Die unterschiedlichen Materialien, die bei dieser Studie zur Herstellung der Kronen verwendet wurden, konnten im Zuge der Auswertung der Ergebnisse zum Teil miteinander verglichen werden. 10 Korrektur- und 10 Doppelmischabformungen wurden jeweils von demselben Dentallabor weiter verarbeitet und es wurden jeweils zwei vollkeramische und eine goldhaltige Restauration hergestellt. In allen Vergleichen schnitten die Keramikrestaurationen besser $\mathrm{ab}$, als die auf der goldhaltigen Legierung basierenden. Da aber zwischen den Ergebnissen für Keramik und Gold keine signifikanten Unterschiede bestanden, und die Differenzen maximal $22 \mu \mathrm{m}$, meist aber sogar unter $15 \mu \mathrm{m}$ betrugen, kann man hier nicht von klinisch relevanten Ergebnissen sprechen.

Mehrere Autoren untersuchten ebenfalls die Passgenauigkeit von Vollkeramikkronen basierend auf CAD/CAM-Techniken. Kokubo zum Beispiel erhob Werte von im Mittel 66 $\mu \mathrm{m}$, Lee

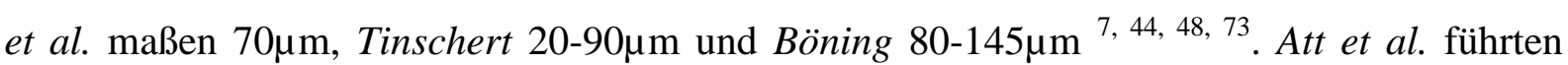
2009 eine Untersuchung zu diesem Thema durch, wobei sie drei verschiedene CAD/CAM Techniken verglichen. Zur Herstellung der Modelle wurden Silikonabformungen durchge- 
führt ${ }^{2}$. Die Ergebnisse im Bezug auf die marginale Genauigkeit vor Zementierung der Kronen betrugen $64-86 \mu \mathrm{m}$. Diese Werte waren zwar höher als die vorliegend gemessenen (21$60 \mu \mathrm{m})$, liegen aber auch in dem Bereich der, wie bereits erwähnt in anderen Studien gemessenen Abweichungen. Somit bestätigen die Resultate der hier präsentierten Studie nochmals die bereits veröffentlichten Ergebnisse anderer Autoren in diesem Themenbereich.

\subsubsection{Diskussion der Ergebnisse des Vergleiches der Zahntechnischen Labora- torien}

Dieser Aspekt wurde bisher noch in keiner anderen Veröffentlichung berücksichtigt. Aus diesem Grund wurde im Rahmen der aktuellen Untersuchung geprüft, ob es im Herstellungsprozess von Kronen, basierend auf der digitalen Abformung, zu relevanten Unterschieden kam, wenn der identischen Scan von zwei verschiedenen Laboratorien weiter bearbeiten wurde.

Die mit dem LCOS aufgenommenen Datensätze wurden an zwei unterschiedliche Laboratorien versendet. Bei den ermittelten Formfehlern sowie bei den absoluten marginalen Diskrepanzen konnten keine signifikanten Unterschiede festgestellt werden. Die Formfehler der Kronen aus dem Labor B waren etwas geringer als die der Kronen aus dem Labor C (Differenz $=11 \mu \mathrm{m})$. Bei der absoluten marginalen Diskrepanz waren die gemessenen Werte der Kronen aus dem Labor $\mathrm{C}$ geringer (Differenz $=15 \mu \mathrm{m}$ ). Aufgrund der fehlenden Vergleichbarkeit zu anderen Studien, gehen wir bei den festgestellten Abweichungen davon aus, dass diese durch Unterschiede in der digitalen Nachbearbeitung oder durch den Fräsvorgang entstanden sind. Die hergestellten Kronen bestanden nur aus den LAVA-Gerüsten, wodurch weitere Einflussfaktoren durch das Aufbringen einer Verblendung bewusst ausgeschlossen wurden. 


\section{$7 \quad$ Schlussfolgerung}

Die auf den digitalen Abformungen beruhenden Kronen wiesen insgesamt vergleichbare Werte bei den untersuchten Parametern im Vergleich zu den konventionellen Kronen auf. Besonders im Hinblick auf die marginale Genauigkeit zeigt sich bei der digitalen Abformung ein großes Potential. Die gemessenen Diskrepanzen lagen mit 26-48 $\mu \mathrm{m}$ in einem sehr guten Bereich.

Bei der Betrachtung der Wiedergabe der inneren Geometrie wäre allerdings teilweise eine genauere Reproduktion wünschenswert. Besonders die auf den CEREC-Scans basierenden Kronen fielen durch hohe Werte im Vergleich $\mathrm{zu}$ allen anderen Kronen auf. Interessanter Weise waren die gemessenen Formfehler bei LCOS am geringsten, sodass sich für die digitale Abformung auch bei der Betrachtung des Formfehlers insgesamt ein mit den konventionellen Techniken vergleichbares Ergebnis zeigt.

Der Vergleich der verschiedenen Restaurationswerkstoffe und zahntechnischen Laboratorien zeigte keine klinisch relevanten Unterschiede.

Betrachtet man die erzielten Ergebnisse unter klinischen Gesichtspunkten, so zeigt sich, dass die erreichten Werte im Bereich der marginalen Diskrepanz die Anforderungen an die erreichbare Genauigkeit absolut erfüllen und sogar übertreffen. Da die hier präsentierte Untersuchung in vitro durchgeführt wurde, ist eine Übertragung auf die klinische Situation natürlich nicht uneingeschränkt möglich. Die limitierenden Faktoren wie Sulkusblutungen oder subgingival gelegene Präparationsgrenzen konnten dabei nicht berücksichtigt werden. Die Untersuchung bezog sich, unter bewusst geschaffenen Idealbedingungen, rein auf den Vergleich der unterschiedlichen Verfahren. 


\section{Zusammenfassung}

Ziel der hier präsentierten Studie war es, die Passgenauigkeit von Kronen, die einerseits auf der Basis klassischer Abformtechniken hergestellt und andererseits via intraoraler Scannverfahren übertragen und gefertigt wurden, zu vergleichen. Zusätzlich wurden die unterschiedlichen verwendeten Restaurationswerkstoffe und die zur Anfertigung der Prüfkörper beauftragten Labore untereinander verglichen.

Zur Durchführung der Studie wurde zunächst ein Urmodell aus Edelstahl angefertigt. Der Zahnkranz wurde in Gips gefasst; Zahn 16 stellte den Urstumpf dar. Basierend auf der zuvor angefertigten Kronenpräparation an einem KaVo-Übungsmodell wurde der Urstumpf in einer Präzisionswerkstatt gefräst. Er bestand aus einem Metallpfeiler, der mit einer Hohlkehlpräparationsgrenze versehen war. Die zirkulär angrenzenden, $2 \mathrm{~mm}$ planparallelen Flächen dienten zur Durchführung der Messungen. Der Stumpf lief oberhalb diese Bereichs nach okklusal hin konisch $\left(6^{\circ}\right.$ Konuswinkel) zusammen. Ein zweiter Urstumpf mit einer matten Oberfläche wurde zur Durchführung der Scans nach identischen Kriterien angefertigt. Mittels einer Verschraubung auf der Modellunterseite konnten die Urstümpfe ausgetauscht werden. Für den Gegenkiefer wurden ebenfalls Zähne aus Edelstahl in Gips gefasst. Das Oberkiefer-Urmodell und das Unterkiefer-Urmodell wurden in zentraler Okklusion einartikuliert. Für die Herstellung der Prüfkörper wurden zuerst 40 Korrektur- und 10 Doppelmischabformungen durchgeführt. Es wurden 90 Kronen von drei verschiedenen zahntechnischen Laboren angefertigt (Nacera Zirkon $n=30$; LAVA Zirkon $n=20$; EMF-Legierung $n=10$; goldhaltige Legierung $\mathrm{n}=30$ ). Im Anschluss wurden 10 Scans mit dem LCOS-Scanner und 20 Scans mit der CEREC-Bluecam durchgeführt. Zwei der Labore fertigten auf deren Basis Kronen (LAVA Zirkon $n=20$; Empress CAD n= 10). Im Eigenlabor unserer Poliklinik wurden weitere 10 Kronen auf Basis von 10 CEREC-Scans angefertigt (Vita Mark II n=10).

Die Prüfkörper wurden nach zwei Kriterien untersucht. Zuerst wurde mit Hilfe einer 3D Koordinaten-Messmaschine (CNC Rapid, THOME GmbH, Messel, Messmaschinennummer: P 654203, Baujahr: 04/ 2007) die interne Wiedergabegenauigkeit, der sogenannte resultierende „Formfehler“ im Vergleich zum Urstumpf ermittelt. Der zweite Untersuchungsparameter war die absolute marginale Diskrepanz, die nach der von Holmes $1989{ }^{32}$ publizierten Methode 
unter einem Messmakroskop M420 (Leica, Bensheim, 20 bis 80fache Vergrößerung) bestimmt wurde.

Zur Durchführung der statistischen Auswertung wurde die SPSS Software, Version 15.0 (SPSS GmbH Software, München, Deutschland) verwendet. Im Anschluss an die varianzanalytische Auswertung wurde der Games-Howel-Test durchgeführt.

Die Ergebnisse der ermittelten Formfehler ergaben die signifikant höchsten Abweichungen vom Urstumpf für die Kronen auf Basis der CEREC-Scans. Alle anderen Prüfgruppen wiesen ähnliche Werte auf. Die absoluten marginalen Diskrepanzen waren bei den CEREC-ScanKronen signifikant geringer als bei den Kronen auf Basis von Korrekturabformungen. Zwischen den Kronen aus LCOS-Scan und CEREC-Scan bestand kein signifikanter Unterschied. Insgesamt kann man sagen, dass die Genauigkeit der digitalen Abformungen in dieser Studie vergleichbar mit der Genauigkeit der konventionellen Abformtechniken war. Besonders vielversprechend sind die Ergebnisse im Bereich der absoluten marginalen Diskrepanz, wogegen im Bezug auf die Wiedergabegenauigkeit der internen Geometrie noch Verbesserungsbedarf besteht. Weitere, vor allem klinische Studien müssen folgen, um das Potential der digitalen Abformung als Alternative zu den konventionellen Techniken hinreichend beurteilen zu können. Besonders an der noch bestehenden Schwierigkeit der Darstellung subgingivaler Bereiche muss auch von Seiten der Entwickler weiter gearbeitet werden, um die Vorstellung von einer ,abformfreien Zahnarztpraxis“ vielleicht in Zukunft Realität werden zu lassen. 


\section{Summary}

The purpose of this study was to evaluate the marginal accuracy and internal roundness of crowns fabricated from intraoral digital impressions in comparison of conventional crowns based on traditional impressions. A steel model of a fully dentate upper jaw was made. The first upper right molar was prepared as a master cast with a chamfer margin, a circular parallel area of $2 \mathrm{~mm}$ and a conical $\left(6^{\circ}\right)$ area of $2.5 \mathrm{~mm}$ on top. 40 impressions of the model were taken in a two-step and 10 impressions in one-step putty-wash impression technique. In three different dental laboratories, 90 conventional crowns were fabricated using different restorative materials (low gold, high gold, ) (Nacera Zirkon $n=30$; LAVA Zirkon $n=20$; cobalt chromium $\mathrm{n}=10$; gold $\mathrm{n}=30$ ). Afterwards 30 scans were taken from the master cast (10x LCOS; $20 \mathrm{X}$ CEREC-Bluecam), and processed in the manufacturer's digital workflow. Two dental laboratories fabricated 30 crowns (LAVA Zirkon $n=20$; Empress CAD $n=10$ ). Furthermore 10 crowns (Vita Mark II) were milled in our proper laboratory.

The internal roundness (major diameter) was accessed and compared to the master using a 3D coordinate measuring machine (CNC Rapid, THOME, Germany). The marginal accuracy of the crowns was accessed with a travelling macroscope (M420 Leitz, Germany) at four predetermined measuring sites on the original master. For statistical analysis the Games-HowellTest was used $(\mathrm{p}<0.05)$.

With regard to internal roundness, crowns based on CEREC-Scan showed significant higher values then all the other crowns. The marginal accuracy of CEREC-Scan crowns was significant better then of the conventionally fabricated (two-step putty-wash technique). There was no significant difference between crowns from LCOS-Scan and CEREC-Scan.

Within the limits of this study it can be concluded, that the intraoral scanning approach has a high potential with regard to the marginal accuracy. Clinical studies are necessary to evaluate to which degree digital impressions have the potential to replace traditional impression techniques. Especially the reproduction of subgingival areas of prepared teeth is an important issue. 


\section{Literaturverzeichnis}

1. Akbar J. H., Petrie C. S., Walker M. P., Williams K., Eick J. D. Marginal adaptation of Cerec $3 \mathrm{CAD} / \mathrm{CAM}$ composite crowns using two different finish line preparation designs. $J$ Prosthodont; 15: 155-163; 2006.

2. Att W., Komine F., Gerds T., Strub J. R. Marginal adaptation of three different zirconium dioxide three-unit fixed dental prostheses. J Prosthet Dent; 101: 239-247; 2009.

3. Bader F., Setz J. [Wettability and accuracy of reproduction of impression materials]. Dtsch Zahnärztl Z; 46: 346-348; 1991.

4. Bader J. D., Rozier R. G., McFall W. T., Jr., Ramsey D. L. Effect of crown margins on periodontal conditions in regularly attending patients. J Prosthet Dent; 65: 75-79; 1991.

5. Beschnidt S. M., Strub J. R. Evaluation of the marginal accuracy of different allceramic crown systems after simulation in the artificial mouth. J Oral Rehabil; 26: 582-593; 1999.

6. Bindl A., Windisch S., Mormann W. H. Full-ceramic CAD/CIM anterior crowns and copings. Int J Comput Dent; 2: 97-111; 1999.

7. Boening K. W., Wolf B. H., Schmidt A. E., Kastner K., Walter M. H. Clinical fit of Procera AllCeram crowns. J Prosthet Dent; 84: 419-424; 2000.

8. Böttger H. Erfahrungen mit Silikonabformmaterialien. Zahnärztl Welt; 74: 616-619; 1965.

9. Böttger H. Über die Abformung beschliffener Zahnstümpfe für Kronenarbeiten mit dem Doppelabdruckverfahren. Deutsch Zahnärztebl 19: 63-66; 1965.

10. D'Arcy B. L., Omer O. E., Byrne D. A., Quinn F. The reproducibility and accuracy of internal fit of Cerec 3D CAD/CAM all ceramic crowns. Eur J Prosthodont Restor Dent; 17: 73-77; 2009.

11. Da Costa J. B., Pelogia F., Hagedorn B., Ferracane J. L. Evaluation of different methods of optical impression making on the marginal gap of onlays created with CEREC 3D. Oper Dent; 35: 324-329; 2010. 
12. Donath K., Roth, K. Histologisch- morphologische Studie zur Bestimmung des zervikalen Randschlusses von Einzel und Pfeilerkronen. Dtsch Zahnärztl Z; 26: 700-705; 1987.

13. Dreyer Jörgensen K. Prüfungsergebnisse zahnärztlicher Gussverfahren Dtsch Zahnärztl Z; 7: 461-469; 1958.

14. Edelhoff D. Digital Dentalnews; 4: 3; 2010.

15. Ender A., Mehl, A. Full arch scans: conventional versus digital impressions--an in vitro study. Int J Comput Dent; 14(1): 11-21; 2011.

16. Fasbinder D. J. The CEREC system: 25 years of chairside CAD/CAM dentistry. $J$ Am Dent Assoc; 141 Suppl 2: 3S-4S.

17. Fasbinder D. J. A clinical evaluation of chairside lithium disilicate CAD/CAM crowns. J Am Dent Assoc; 141: 2105-2145); 2010.

18. Fasbinder D. J., Poticny D. J. Accuracy of occlusal contacts for crowns with chairside CAD/CAM techniques. Int J Comput Dent; 13: 303-316; 2010.

19. Fejér S. Das Röntgenbild als Kontrollmöglichkeit des approximal cervikalen Randschlusses bei Gussfüllungen an Prämolaren und Molaren. Dtsch Zahnärztl Z; 11: 1086- 1091; 1956.

20. Fenske C. Sadat-Khonsari M. R., Jüde H. D. Der Einfluss verschiedener Abformtechniken auf die Dimensionstreue von Modellstümpfen. Dtsch Zahnärztl Z 56: 35-38; 2001.

21. Fenske C., Sadat-Khonsari, M. R., Jüde, H. D. Der Einfluss verschiedener Abformtechniken auf die Dimensionstreue von Modellstümpfen. Dtsch Zahnärztl Z 56: 35-38; 2001.

22. Fransson B., Oilo G., Gjeitanger R. The fit of metal-ceramic crowns, a clinical study. Dent Mater; 1: 197-199; 1985.

23. Frigerio F. 3 -Dimensional Surface Imagine Using Active Wavefront Sampling. Med Diss. Massachusetts; 2006.

24. Gall H. Allergische Reaktionen auf zahnärztliche Werkstoffe. Dtsch Zahnärztl Z; 38: $735-741 ; 1983$.

25. Geurtsen W. [Crown and restoration margins]. Dtsch Zahnärztl Z; 45: 380-386; 1990.

26. Grasso J. E., Nalbandian J., Sanford C., Bailit H. Effect of restoration quality on periodontal health. J Prosthet Dent; 53: 14-19; 1985. 
27. Haas M., Wegscheider W. A., Bratschko R. O., Pfeiffer K., Kuderna F. [Comparison of margin in metal and non-metal ceramic crowns]. Quintessenz; 39: 625-633; 1988.

28. Haeusler G. Über die kluge Auswahl und Anwendung optischer 3D-Sensoren. Photonik; 5: 2-5; 2004.

29. Hirschfeder C., Grüner, M., Stark, H., Helfgen, E-H.,. Dimensionsstabilität von Abdrucklöffeln und -material mit Blick auf die offene Implantatabformung. Dtsch Zahnärztl Z; 63: 418- 423; 2008.

30. Hoffmann -Axthelm W. Die Geschichte der Zahnheilkunde. Berlin: Quintessenz 1973.

31. Hofmann M. [the Corrective Impression. (a New Impression Technic for Fixed Dentures)]. Zahnärztl Welt Zahnärztl Reform Zwr; 66: 160-164; 1965.

32. Holmes J. R., Bayne S. C., Holland G. A., Sulik W. D. Considerations in measurement of marginal fit. J Prosthet Dent; 62: 405-408; 1989.

33.

http://solutions.3mdeutschland.de/wps/portal/3M/de_DE/3M_ESPE/DentalManufactur ers/; $(12.10 .2011)$

34. http://www.cad-programme.com/tipps/rundheit; (6.11.2011)

35. http://www.cerec.connect.de/ecomaXL/; (12.10.2011)

36. http://www.meditec.hia.rwth-aachen.de/forschung/aktuell-projekte/ida.html;

$(21.01 .2012)$

37. http://www.straumann-cares-digital-solutions.com/com-index/digitalsolutions/intraoral-scanning/intraoral-scanning-models.htm; (12.10.2011)

38. Jacobs M. S., Windeler A. S. An investigation of dental luting cement solubility as a function of the marginal gap. J Prosthet Dent; 65: 436-442; 1991.

39. Kachalia P. R., Geissberger M. J. Dentistry a la carte: in-office CAD/CAM technology. J Calif Dent Assoc; 38: 323-330; 2010.

40. Kerschbaum T.H. Porschen C. Kronenrandschluss und- Kontinuität in fünf Dentallaboratorien. Dtsch Zahnärztl Z; 53: 620-623; 1998.

41. Kerschbaum T.H., Porschen, C. . Kronenrandschluss und- Kontinuität in fünf Dentallaboratorien. Dtsch Zahnärztl Z; 53: 620-623; 1998. 
42. Klein B. Rundheit/ Form und Lagetoleranzen. Toleranzmanagement im Maschinen und Fahrzeugbau, Form- und Lagetoleranzen, Toleranzprinzipien, Tolerierungsverknüpfungen, Maßketten, Oberflächen. Oldenbourg Wissenschaftsverlag 2005.

43. Knoblauch M. [Experiences with the double impression method (corrective impression)]. Dtsch Zahnärztl Z; 21: 169-170; 1966.

44. Kokubo Y., Nagayama Y., Tsumita M., Ohkubo C., Fukushima S., Vult von Steyern P. Clinical marginal and internal gaps of In-Ceram crowns fabricated using the GN-I system. J Oral Rehabil; 32: 753-758; 2005.

45. Körber E. Zahnärztliche Prothetik. 3. Stuttgart: Thieme 1985.

46. Kulenkamp D., Hausen, B.M., Schulz, K.-H. Berufliche Kontaktallergie durch neuartige Abdruckmaterialien in der zahnärztlichen Praxis. Zahnärztl Mitt; 66: 968-969; 1976.

47. Laurent M., Scheer P., Dejou J., Laborde G. Clinical evaluation of the marginal fit of cast crowns--validation of the silicone replica method. J Oral Rehabil; 35: 116-122; 2008.

48. Lee K. B., Park C. W., Kim K. H., Kwon T. Y. Marginal and internal fit of all-ceramic crowns fabricated with two different CAD/CAM systems. Dent Mater J; 27: 422-426; 2008.

49. Lehmann K. M., Burgdorf H. O. [2-stage impression technic for crowns and bridgework (corrective impression)]. ZWR; 87: 430-433; 1978.

50. Lehmann K. M., Burgdorf H. O. [Single-stage impression technic for crowns and bridgework (double mix impression)]. ZWR; 87: 434-436; 1978.

51. Luthardt R. G., Loos R., Quaas S. Accuracy of intraoral data acquisition in comparison to the conventional impression. Int J Comput Dent; 8: 283-294; 2005.

52. Marxkors R. Kriterien für die zahnärztliche Prothetik. Studienbuch des Projekts Qualitätssicherung in der Zahnmedizin. Würzburg: 1988: 46.

53. Marxkors R. Lehrbuch der zahnärztlichen Prothetik. Wien: Hanser München 1991.

54. Marxkors R. [Marginal seal of cast crowns]. Dtsch Zahnärztl Z; 35: 913-915; 1980.

55. Marxkors R., Meiners, H. Taschenbuch der zahnärztlichen Werkstoffkunde. 3. München: Hanser 1988.

56. Mehl A., Ender A., Mormann W., Attin T. Accuracy testing of a new intraoral 3D camera. Int J Comput Dent; 12: 11-28; 2009. 
57. Mormann W. H. The evolution of the CEREC system. J Am Dent Assoc; 137: 7-13; 2006.

58. Nissan J., Laufer B. Z., Brosh T., Assif D. Accuracy of three polyvinyl siloxane puttywash impression techniques. J Prosthet Dent; 83: 161-165; 2000.

59. Peutzfeldt A., Asmussen, E. Accuracy of alginate and elastomeric impression materials. Scand J Dent Res; 97: 74-81; 1989.

60. Pfeifer T., Schmitt, R. Rundheitstoleranz. Fertigungsmesstechnik. 3. Oldenbourg Wissenschaftsverlag 2010.

61. Poller A. Das Pollersche Verfahren zum Abformen an Lebenden und Toten sowie an Gegenständen. Berlin- Wien: Urban \& Schwarzenberg 1931.

62. Rehberg H. J. [Exact marginal closure--what's that?]. Dtsch Zahnärztl Z; 26: 696-699; 1971.

63. Reich S. M., Wichmann M., Rinne H., Shortall A. Clinical performance of large, allceramic CAD/CAM-generated restorations after three years: a pilot study. J Am Dent Assoc; 135: 605-612; 2004.

64. Riehl J., jr.Train Serv, DV- Training,-- Service, Markt-/ Sozialforschung Giessen. Abformgenauigkeit und Abformmethode. 2011.

65. Rudolph H., Quaas, S., Luthardt, RG. Matching point Clouds: Limits and Possibilities [Über das Zusammensetzen (Matchen) von Punktewolken: Grenzen und Möglichkeiten]. Int J Comput Dent; 5: 155-164; 2002.

66. Sadat Khonsari M.R. Fenske C., Jüde H. D., Gütschow F. Untersuchung zur Korrekturabformung.

67. Schulz H.H., Schwickerath, H. Die Abformung in der Zahnheilkunde. Köln: Deutscher Ärzte-Verlag 1989.

68. Spiekermann H. [The marginal fit of crowns and bridges]. Dtsch Zahnärztl Z; 41: 1015-1019; 1986.

69. Stahl E. Präzise Abdrücke nach dem Doppelabdruckverfahren speziell für stufenlose Präparationenund Herstellung exakter Modelle für indirekte Kronen-Brücken und Inlayarbeiten. Dtsch Zahnärztebl 10: 46-52 1956. 
70. Suárez M.J. Comparison of the Marginal Fit of Procera AllCeram Crowns with Two Finish Lines. The Int Journal of Prosthodontics; 16: 229-232; 2003.

71. Sulaiman F., Chai J., Jameson L. M., Wozniak W. T. A comparison of the marginal fit of In-Ceram, IPS Empress, and Procera crowns. Int J Prosthodont; 10: 478-484; 1997.

72. Syrek A., Reich G., Ranftl D., Klein C., Cerny B., Brodesser J. Clinical evaluation of all-ceramic crowns fabricated from intraoral digital impressions based on the principle of active wavefront sampling. J Dent; 38: 553-559; 2010.

73. Tinschert J., Natt G., Mautsch W., Spiekermann H., Anusavice K. J. Marginal fit of alumina-and zirconia-based fixed partial dentures produced by a CAD/CAM system. Oper Dent; 26: 367-374; 2001.

74. Tsitrou E. A., Northeast S. E., van Noort R. Evaluation of the marginal fit of three margin designs of resin composite crowns using CAD/CAM. J Dent; 35: 68-73; 2007.

75. Viohl J. Abformwerkstoffe. In: Eichner K (Hrsg.). Zahnärztliche Werkstoffe und ihre Verarbeitung. Heidelberg: Hüthig 1981.

76. Weaver J. D., Johnson G. H., Bales D. J. Marginal adaptation of castable ceramic crowns. J Prosthet Dent; 66: 747-753; 1991.

77. Wichmann M. Kronen und Brücken. Dtsch Zahnärztl Z; 54: 469- 470; 1999.

78. Wichmann M., Borchers L., Limmroth E. [Measuring the accuracy of various elastomeric impression materials using a CNC coordinate measuring device (Part 1)]. Dtsch Zahnärztl Z; 45: 499-502; 1990.

79. Wöstmann B., Dohle A, Rettberg S. Zur Frage der in vivo erreichbareb Abformgenauigkeit. Dtsch Zahnärztl Z; 49: 679-682; 1994.

80. Wöstmann B. Die Abformung als Grundlage für eine langfristige prothetische Versorgung. Zahnärztl Mitt 95: 2856-2863; 2005.

81. Wöstmann B. Die Abformung präparierter Zahnhartsubstanz.. Zahnärztl Mitt 89: 1216-1225; 1999.

82. Wöstmann B. Zum derzeitigen Stand der Abformung in der Zahnheilkunde. . Quintessence Verlags- GmbH Berlin 1998.

83. Wöstmann B. Hönig M., Ferger P. Vergleich von hand und maschinengemischten Abformmassen(Pentamix System). Dtsch ZahnärztlZ 53: 753-756; 1998. 
84. Wöstmann B., Koniaris, A., Ferger, P. Der Einfluss von Feuchtigkeit auf die Abformgenauigkeit in einem Sulkus-Fluid-Flow-Modell. . Dtsch Zahnärztl Z; 54: 631-633 1999.

85. Wöstmann B., Symeonidis, A., Ferger, P. Abformgenauigkeit und Lagerungsstabilität moderner C-Silikone. Dtsch Zahnärztl Z; 54: 634-636; 1999. 


\section{Anhang}

\subsection{Tabellenverzeichnis}

Tabelle 1: $\quad$ Übersicht über die verwendeten Materialien bei den Silikon-

Abformungen

Tabelle 2: $\quad$ Hersteller der Prüfkörper

Tabelle 3: $\quad$ Überblick über die Herstellung der Prüfkörper

Tabelle 4: $\quad$ Scan/ Herstellungsparameter Nacera Zirkon Kronen

Tabelle 5: $\quad$ Scan/ Herstellungsparameter für LAVA Zirkon

Tabelle 6: $\quad$ Scan/ Herstellungsparameter für IPS Empress CAD

Tabelle 7: $\quad$ Scan/ Herstellungsparameter für Vita Mark II

Tabelle 8: $\quad$ Überblick über die vorhandenen Daten

Tabelle 9: $\quad$ Der Formfehler in den Vergleichsgruppen

Tabelle 10: $\quad$ Absolute marginale Diskrepanz in den Vergleichsgruppen

Tabelle 11: $\quad$ Formfehler von LAVA Zirkonkronen bei unterschiedlichen Abformtechniken

Tabelle 12: $\quad$ Absolute marginale Diskrepanz von LAVA Zirkonkronen bei unterschiedlichen Abformtechniken

Tabelle 13: $\quad$ Darstellung der verschiedenen Materialen im Vergleich bei sonst gleichen Parametern (Korrekturtechnik)

Tabelle 14: $\quad$ Darstellung der verschiedenen Materialen im Vergleich bei sonst gleichen Parametern (Doppelmischtechnik)

Tabelle 15: $\quad$ Darstellung der verschiedenen Materialen im Vergleich bei sonst gleichen Parametern (Korrekturtechnik)

Tabelle 16: $\quad$ Darstellung der verschiedenen Materialen im Vergleich bei sonst gleichen Parametern (Doppelmischtechnik) 


\subsection{Abbildungsverzeichnis}

Abb. 1: Definition des Randschlusses nach Donath und Roth.

Abb. 2: $\quad$ Absolute marginale Diskrepanz nach Holmes et al.

Abb. 3: $\quad$ Formen des Randschlusses nach Holmes et al.

Abb. 4: $\quad$ Schematische Darstellung des Urstumpfes

Abb. 5: $\quad$ Urstumpf 16

Abb. 6: $\quad$ Oberkiefermodell mit Urstumpf 16

Abb. 7: $\quad$ Detailaufnahme Scan-Urstumpf

Abb. 8: $\quad$ Urstumpf mit matter Oberfläche

Abb. 9: $\quad$ Scanvorgang mit LCOS

Abb. 10: $\quad$ Urmodell in zentraler Okklusion

Abb. 11: $\quad$ Schemazeichnung Rundheit

Abb. 12: $\quad$ Krone zur Vermessung platziert/ Taster im Lumen

Abb. 13: $\quad 3 D-$ Koordinaten- Messmaschine

Abb. 14: Absolute marginale Diskrepanz nach Holmes et al.

Abb. 15: Krone auf Urstumpf

Abb. 16: $\quad$ Messmakroskop M420

Abb. 17: $\quad$ Boxplot Diagramm der Formfehler aller Kronen

Abb. 18: $\quad$ Boxplot Diagramm der absoluten marginalen Diskrepanz aller Kronen

Abb. 19: Boxplot Diagramm des Formfehlers bezogen auf die unterschiedlichen Abformverfahren

Abb. 20: Boxplot Diagramm der absoluten marginalen Diskrepanz bezogen auf die unterschiedlichen Abformverfahren 


\section{Erklärung}

„Hiermit erkläre ich, dass ich die vorliegende Arbeit selbständig und ohne unzulässige Hilfe oder Benutzung anderer als der angegebenen Hilfsmittel angefertigt habe. Alle Textstellen, die wörtlich oder sinngemäß aus veröffentlichten oder nichtveröffentlichten Schriften entnommen sind, und alle Angaben, die auf mündlichen Auskünften beruhen, sind als solche kenntlich gemacht. Bei den von mir durchgeführten und in der Dissertation erwähnten Untersuchungen habe ich die Grundsätze guter wissenschaftlicher Praxis, wie sie in der „Satzung der Justus-Liebig-Universität Gießen zur Sicherung guter wissenschaftlicher Praxis“ niedergelegt sind, eingehalten sowie ethische, datenschutzrechtliche und tierschutzrechtliche Grundsätze befolgt. Ich versichere, dass Dritte von mir weder unmittelbar noch mittelbar geldwerte Leistungen für Arbeiten erhalten haben, die im Zusammenhang mit dem Inhalt der vorgelegten Dissertation stehen, oder habe diese nachstehend spezifiziert. Die vorgelegte Arbeit wurde weder im Inland noch im Ausland in gleicher oder ähnlicher Form einer anderen Prüfungsbehörde zum Zweck einer Promotion oder eines anderen Prüfungsverfahrens vorgelegt. Alles aus anderen Quellen und von anderen Personen übernommene Material, das in der Arbeit verwendet wurde oder auf das direkt Bezug genommen wird, wurde als solches kenntlich gemacht. Insbesondere wurden alle Personen genannt, die direkt und indirekt an der Entstehung der vorliegenden Arbeit beteiligt waren. Mit der Überprüfung meiner Arbeit durch eine Plagiatserkennungssoftware bzw. ein internetbasiertes Softwareprogramm erkläre ich mich einverstanden.“ 


\section{Danksagung}

Ich bedanke mich bei

Prof. Dr. Bernd Wöstmann für die freundliche Überlassung des Themas und die Unterstützung bei der Verfassung und Ausführung meiner Dissertation.

MTA Ulrich Heun der mir während der Vermessung der Kronen im werkstoffkundlichen Labor stets mit Rat und Tat zur Seite stand.

Dr. Jürgen Riehl für die Unterstützung bei der statistischen Auswertung der Daten.

Dr. Paul Seelbach für seine Unterstützung bei der Durchführung der digitalen Abformungen und der Formatierung dieser Arbeit.

Dr. Peter Rehmann der mir bei allen Fragen stets hilfsbereit zur Seite stand und mir beim Korrekturlesen eine sehr große Hilfe war.

Meinen Eltern, Großeltern und meinem Freund Stephan Leitner für ihr Verständnis, Vertrauen und ihre stets liebevolle Unterstützung während meines gesamten Studiums und meiner Promotionsphase. 